SLAC-PUB-11687

LBNL-59634

February, 2006

hep-ph/yymmnnn

\title{
Determination of Dark Matter Properties at High-Energy Colliders
}

\author{
EDWARD A. BALTZ ${ }^{1}$ \\ Kavli Institute for Particle Astrophysics and Cosmology \\ Stanford University, Stanford, California 94309 USA \\ MARCo BATTAGLiA ${ }^{2}$ \\ Department of Physics and Lawrence Berkeley Laboratory \\ University of California, Berkeley, California 94720 USA \\ Michael E. Peskin and Tommer Wizansky ${ }^{1}$ \\ Stanford Linear Accelerator Center \\ Stanford University, Stanford, California 94309 USA
}

\begin{abstract}
If the cosmic dark matter consists of weakly-interacting massive particles, these particles should be produced in reactions at the next generation of high-energy accelerators. Measurements at these accelerators can then be used to determine the microscopic properties of the dark matter. From this, we can predict the cosmic density, the annihilation cross sections, and the cross sections relevant to direct detection. In this paper, we present studies in supersymmetry models with neutralino dark matter that give quantitative estimates of the accuracy that can be expected. We show that these are well matched to the requirements of anticipated astrophysical observations of dark matter. The capabilities of the proposed International Linear Collider (ILC) are expected to play a particularly important role in this study.
\end{abstract}

\footnotetext{
${ }^{1}$ Work supported by the US Department of Energy, contract DE-AC02-76SF00515.

${ }^{2}$ Work supported by the US Department of Energy, contract DE-AC02-05CH11231.
} 


\section{Contents}

1 Introduction 1

2 Preliminaries 3

2.1 Why the WIMP model of dark matter deserves special attention . . . 3

2.2 WIMPs at high-energy colliders . . . . . . . . . . . . . 5

2.3 Qualitative determination of WIMP parameters . . . . . . . . . 7

2.4 Quantitative determination of WIMP parameters . . . . . . . . . 9

2.5 Astrophysical dark matter measurements: relic density . . . . . . . . 10

2.6 Astrophysical dark matter measurements: direct detection . . . . . . 11

2.7 Astrophysical dark matter measurements: WIMP annihilation . . . . 14

2.8 Summary: steps toward an understanding of WIMP dark matter . . . 18

3 Models of neutralino dark matter $\quad 19$

3.1 Mechanisms of neutralino annihilation f . . . . . . . . . . . 19

3.2 Choice of benchmark models . . . . . . . . . . . . . . . . . . 21

3.3 Scanning of parameter space . . . . . . . . . . . . . 25

3.4 Parameters and constraints . . . . . . . . . . . . . . 26

3.5 Importance sampling . . . . . . . . . . . . . . . . . 28

4 Benchmark point LCC1 29

4.1 Spectroscopy measurements . . . . . . . . . . . . . . . 31

4.2 Relic density . . . . . . . . . . . . . . . . . . . 34

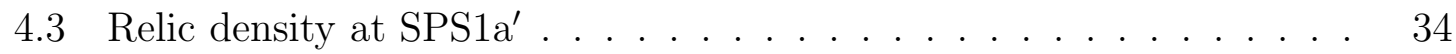

4.4 Annihilation cross section . . . . . . . . . . . . . . . 38

4.5 Direct detection cross section . . . . . . . . . . . . . . . 43

4.6 Constraints from relic density and direct detection . . . . . . . . . . . 48

5 Benchmark point LCC2 51 
5.1 Spectroscopy measurements . . . . . . . . . . . . . . 51

5.2 Relic density . . . . . . . . . . . . . . . . . . . . . . 53

5.3 Annihilation cross section $\ldots \ldots \ldots \ldots \ldots \ldots$

5.4 Direct detection cross section $\ldots \ldots \ldots \ldots$. . . . . . . . 56

5.5 Constraints from relic density and direct detection . . . . . . . . 63

6 Benchmark point LCC3 $\quad 63$

6.1 Spectroscopy measurements . . . . . . . . . . . . . . . . . 64

6.2 Relic density . . . . . . . . . . . . . . . . . . . . . 68

6.3 Annihilation cross section $\ldots \ldots \ldots$. . . . . . . . . . . . 69

6.4 Direct detection cross section $\ldots \ldots \ldots \ldots \ldots$

6.5 Constraints from relic density and direct detection . . . . . . . . . 73

$\begin{array}{lll}7 & \text { Benchmark point LCC4 } & 77\end{array}$

7.1 Spectroscopy measurements . . . . . . . . . . . . . . . 77

7.2 Relic density . . . . . . . . . . . . . . . . . . 80

7.3 Annihilation cross section $\ldots \ldots \ldots \ldots \ldots$

7.4 Direct detection cross section $\ldots \ldots \ldots \ldots$. . . . . . . 84

7.5 Constraints from relic density and direct detection . . . . . . . . 87

8 Neutralino annihilation products $\quad 88$

8.1 Gamma ray spectra . . . . . . . . . . . . . . 88

8.2 Positron spectra . . . . . . . . . . . . . . . . . . 92

9 Recap: Collider determination of WIMP properties 94

9.1 Summary of results: cross sections . . . . . . . . . . . . . . . . . 94

9.2 Summary of result: astrophysics . . . . . . . . . . . . . 96

9.3 LHC and astrophysical measurements . . . . . . . . . . . . . . . 99

9.4 ILC at $500 \mathrm{GeV} \ldots \ldots \ldots$. . . . . . . . . . . . . . . . . 101 
9.5 ILC at $1000 \mathrm{GeV} \ldots \ldots \ldots \ldots 2$

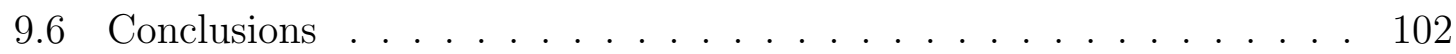

A Markov Chain Monte Carlo 103

A.1 Adaptive Metropolis-Hastings algorithm . . . . . . . . . . . . . 103

A.2 Exploring the distributions . . . . . . . . . . . . 106

A.3 Thinning the chains . . . . . . . . . . . 106

A.4 Convergence test . . . . . . . . . . . . . . . . 109

B Disconnected families of supersymmetry parameters 109

B.1 Benchmark point LCC1 f . . . . . . . . . . . . . . 109

B.2 Benchmark point LCC2 . . . . . . . . . . . . . 110

B.3 Benchmark points LCC3 and LCC4 . . . . . . . . . . . . . . . . 111

Submitted to Physical Review D 


\section{Introduction}

It is now well established that roughly $20 \%$ of the energy density of the universe consists of neutral weakly interacting non-baryonic matter, 'dark matter' [1-3]. The picture of structure formation by the growth of fluctuations in weakly interacting matter explains the elements of structure in the universe from the fluctuations in the cosmic microwave background down almost to the scale of galaxies. However, many mysteries remain. From the viewpoint of particle physics, we have no idea what dark matter is made of. The possibilities range in mass from axions (mass $10^{-5}$ $\mathrm{eV})$ to primordial black holes (mass $10^{-5} M_{\odot}$ ). From the viewpoint of astrophysics, it is still controversial how dark matter is distributed in galaxies and even whether the picture of weakly interacting dark matter adequately explains the structure of galaxies. Furthermore, a much larger range of masses is allowed, bounded only by the quantum limit $\left(10^{-22} \mathrm{eV}\right)$ for bosons [4] and the discreteness limit $\left(10^{3} M_{\odot}\right)$ above which galactic globular clusters would be disrupted by the dark matter "particles" $[5]$.

To improve this situation, we need more experimental measurements. Unfortunately, precisely because dark matter is weakly interacting and elusive, any single new piece of data has multiple interpretations. If we improve the upper limit on the direct detection of dark matter, does this mean that the microscopic cross section is small or that our detector is located in a trough of the galactic dark matter distribution? If we see a signal of dark matter annihilation at the center of the galaxy, does this measure the annihilation cross section, or does it measure the clustering of dark matter associated with the galaxy's formation? If we observe a massive weakly-interacting elementary particle in a high-energy physics experiment, can we demonstrate that this particle is a constituent of dark matter? For any single question, there are no definite answers. It is only by carrying out a program of experiments that include both particle physics and astrophysics measurements and marshalling all of the resulting information that we could reach definite conclusions.

It is our belief that the role that particle physics measurements will play in this program has been underestimated in the literature. Much of the particle astrophysics literature on dark matter particle detection is written as if we should not expect strong constraints from particle physics on the microscopic cross sections. This leaves a confusing situation, in which one needs to determine the basic properties of dark matter in the face of large uncertainties in its galactic distribution, or vice versa. To make matters worse, many of these discussions use models of dark matter with an artificially small number of parameters, precisely to limit the possible modes of variation in the microscopic properties of dark matter.

We see good reason to be more optimistic. Among the many possible models of 
dark matter, we believe that there are strong reasons to concentrate on the particular class of models in which the dark matter particle is a massive neutral particle with a mass of the order of $100 \mathrm{GeV}$. We will refer to the particles in this special class of models as WIMPs. We will define the class more precisely in Section 2. In this class of models, the WIMP should be discovered in high-energy physics experiments just a few years from now at the CERN Large Hadron Collider (LHC). Over the next ten years, the LHC experiments and experiments at the planned International Linear Collider (ILC) will make precision measurements that will constrain the properties of the WIMP. This in turn will lead to very precise determinations of the microscopic cross sections that enter the dark matter abundance and detection rates.

In the best situation, these experiments could apply to the study of dark matter the strategy used in more familiar areas of astrophysics. When we study stars and the visible components of galaxies and clusters, every measurement is determined by a convolution of microscopic cross sections with astrophysical densities. We go into the laboratory to measure atomic and nuclear transition rates and then apply this information to learn the species and conditions in the object we are observing. We might hope that the LHC and ILC experiments on dark matter would provide the basic data for this type of analysis of experiments that observe galactic dark matter.

To satisfy this objective, we need to realistically evaluate the power of the LHC and ILC experiments to determine the cross sections of direct astrophysical interest. Dark matter particles are invisible to high-energy physics experiments, and so such determinations are necessarily indirect. On the other hand, the large number of specific and precise measurements that can be expected will allow us to determine the model of which the WIMP is a part, with sufficient precision to fix the otherwise elusive cross sections. Our goal in this paper is to demonstrate this point, and to estimate the accuracy that can be expected from the LHC and ILC data.

Dark matter measurements also have the potential to feed information back to particle physics. Today, the very existence of dark matter is the strongest piece of evidence for physics beyond the Standard Model. The cosmic density of dark matter is already quite well known. This density has been determined to $6 \%$ accuracy by the cosmological data, especially by the WMAP measurement of the cosmic microwave background (CMB) [6]. Later in this decade, the Planck satellite should improve this determination to the $0.5 \%$ level [7]. If it should become attractive to assume that a WIMP observed at the LHC accounts for all of the dark matter, these measurements can be used to give precision determinations of some particle physics parameters. Over time, this assumption could be tested with higher-precision high-energy physics experiments. In some cases, measurements of direct signals of astrophysical dark matter could also provide interesting constraints on particle physics. We will give some illustrations of this interplay between astrophysical and microscopic constraints 
in the context of our examples.

Here is an outline of our analysis: In Section 2, we will specify the WIMP class of dark matter models, and we will review the set of WIMP properties that should be determined by microscopic experiments. To perform specific calculations of the ability of LHC and ILC to determine these cross sections, we will study in detail the case of supersymmetry models in which the dark matter particle is the lightest neutralino. In Section 3, we will review the various physical mechanisms that can be responsible for setting the dark matter relic density in these models, and we will choose four benchmark models for detailed study. We will also explain how we evaluate the model uncertainty in the predictions from the collider measurements, using an exploration of the parameter space by Markov Chain Monte Carlo techniques. In Sections 4-7, we present the results of our Monte Carlo study for each of the benchmark points. In Section 8, we will present some general observations on the determination of dark matter annihilation cross sections. Finally, in Section 9, we review the results of our study and present the general conclusions that we draw from them.

Our calculations make heavy use of the ISAJET [8] and DarkSUSY computer programs [9] to evaluate the neutralino dark matter properties from the underlying supersymmetry parameters. We thank the authors for making these useful tools available.

The determination of the cosmic dark matter density from collider data has also been studied recently by Allanach, Belanger, Boudjema, and Pukhov [10] and by Nojiri, Polesello, and Tovey [11]. We will compare our strategies and results in Sections 3 and 4. A first version of this analysis has been presented in [12]; this work supersedes the results presented in that paper.

\section{Preliminaries}

Before beginning our study of specific WIMP models of dark matter, we would like to review some general aspects of dark matter and its observation. In this section, we will define what we mean by the WIMP scenario, give an overview of how WIMPs can be studied at high-energy colliders, and review the set of cross sections needed to analyze WIMP detection.

\subsection{Why the WIMP model of dark matter deserves special attention}

Among the particle physics candidates for dark matter, many share a set of common properties. They are heavy, neutral, weakly-interacting particles with interaction cross sections nevertheless large enough that they were in thermal equilibrium 
for some period in the early universe. It is these particles that we refer to collectively as WIMPs.

The assumption of thermal equilibrium allows a precise prediction of the cosmic density of the WIMP. We must of course also assume that standard cosmology can be extrapolated back to this era. Given these assumptions, it is straightforward to integrate the Boltzmann equation for the WIMP density through the time at which the WIMP drops out of equilibrium. The resulting density is the 'relic density' of the WIMP. To $10 \%$ accuracy, the ratio of this relic density to the closure density is given by the formula [13]

$$
\Omega_{\chi} h^{2}=\frac{s_{0}}{\rho_{c} / h^{2}}\left(\frac{45}{\pi g_{*}}\right)^{1 / 2} \frac{x_{f}}{m_{\mathrm{Pl}}} \frac{1}{\langle\sigma v\rangle}
$$

where $s_{0}$ is the current entropy density of the universe, $\rho_{c}$ is the critical density, $h$ is the (scaled) Hubble constant, $g_{*}$ is the number of relativistic degrees of freedom at the time that the dark matter particle goes out of thermal equilibrium, $m_{\mathrm{Pl}}$ is the Planck mass, $x_{f} \approx 25$, and $\langle\sigma v\rangle$ is the thermal average of the dark matter pair annihilation cross section times the relative velocity. Most of these quantities are numbers with large exponents. However, combining them and equating the result to $\Omega_{N} \sim 0.2[1]$, we obtain

$$
\langle\sigma v\rangle \sim 0.9 \mathrm{pb}
$$

Interpreting this in terms of a mass, using $\langle\sigma v\rangle=\pi \alpha^{2} / 8 m^{2}$, we find $m \sim 100 \mathrm{GeV}$.

It is remarkable that this estimate places the mass scale of dark matter just at a place where we expect for other reasons to find new physics beyond the Standard Model of particle physics. Our current understanding of the weak interaction is that this arises from a gauge theory of the group $S U(2) \times U(1)$ that is spontaneously broken at the hundred-GeV energy scale. An astronomer might note this as a remarkable coincidence. A particle theorist would go further. There are many possible, and competing, models of weak interaction symmetry breaking. In any of these models, it is possible to add a discrete symmetry that makes the lightest newly introduced particle stable. Generically, this particle is heavy and neutral and meets the definition of a WIMP that we have given above. In many cases, the discrete symmetry in question is actually required for the consistency of the theory or arises naturally from its geometry. For example, in models with supersymmetry, imposing a discrete symmetry called R-parity is the most straightforward way to eliminate dangerous baryon-number violating interactions. Thus, as particle theorists, we are almost justified in saying that the problem of electroweak symmetry breaking predicts the existence of WIMP dark matter. This statement also has a striking experimental implication, which we will discuss in the next section.

The fact that models of electroweak symmetry breaking predict WIMP dark matter was recognized very early for the important illustrative example of supersymmetry. 
Dark matter was discussed as a consequence of the theory in some of the earliest papers on supersymmetry phenomenology [14-18]. However, it is important to realize that the logic of this connection is not special to supersymmetry; it is completely general. This has been emphasized recently by the introduction of dark matter candidates associated with extra-dimensional and little Higgs models of electroweak symmetry breaking [19-23].

If WIMP dark matter is preferred by theory, it is also preferred by experiment, or at least, by experimenters. Almost every technique that has been discussed for the detection of dark matter requires that the dark matter is composed of heavy neutral particles with weak-interaction cross sections. (The axion search experiment [24] gives the only counterexample.)

The arguments we have given do not rule out dark matter candidates in other mass regions. It might well be true that WIMPs exist as a consequence of our models of weak interaction symmetry breaking, but that they make up only a small part of the dark matter. The only way to find this out is to carry out the experiments that define the properties of the WIMPs, predict their relic density and detection cross sections, and find discrepancies with astrophysical observation. Either way, we must continue to the steps described in the following sections.

\subsection{WIMPs at high-energy colliders}

There is a further assumption that, when added to the properties of a WIMP just listed, has dramatic implications. Models of electroweak symmetry breaking typically contain new heavy particles with QCD color. These appear as partners of the quarks to provide new physics associated with the generation of the large top quark mass. In supersymmetry and in many other models, electroweak symmetry breaking arises as a result of radiative corrections due to these particles, enhanced by the large coupling of the Higgs boson to the top quark. Thus, we would like to add to the structure of WIMP models the assumption that there exists a new particle that carries the conserved discrete symmetry and couples to QCD. This particle should have a mass of the same order of magnitude as the WIMP, below $1000 \mathrm{GeV}$.

Any particle with these properties will be pair-produced at the CERN Large Hadron Collider (LHC) with a cross section of tens of pb. The particle will decay to quark or gluon jets and a WIMP that exits a particle physics detector unseen. Thus, any model satisfying these assumptions predicts that the LHC experiments will observe events with many hadronic jets and an imbalance of measured momentum. These 'missing energy' events are well-known to be a signature of models of supersymmetry. In fact, they should be seen in any model (subject to the assumption just given) that contains a WIMP dark matter candidate. 


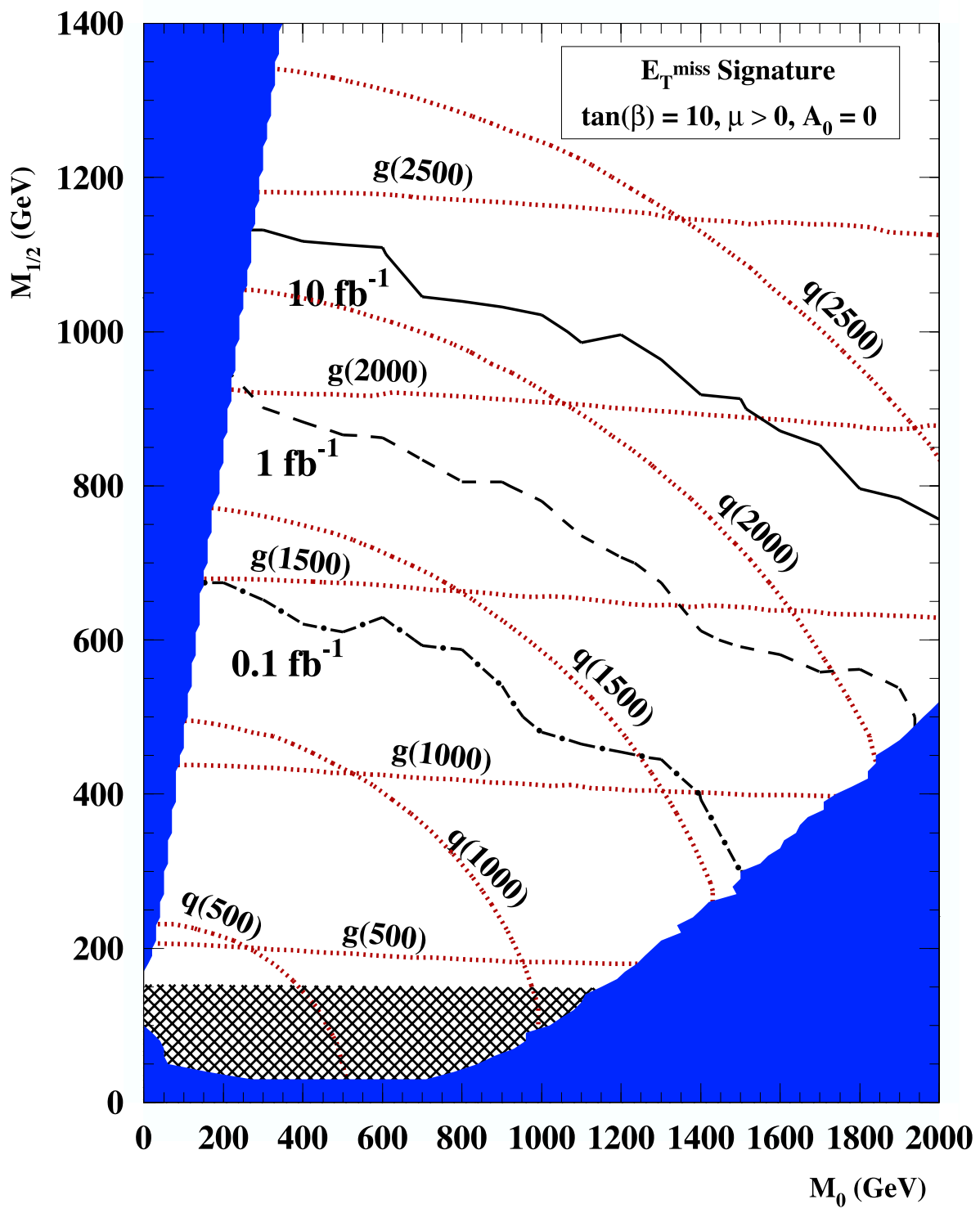

Figure 1: Contours in a parameter space of supersymmetry models for the discovery of the missing energy plus jets signature of new physics by the ATLAS experiment at the LHC. The three sets of contours correspond to levels of integrated luminosity at the LHC (in $\left.\mathrm{fb}^{-1}\right)$, contours of constant squark mass, and contours of constant gluino mass. From [25]. 
The rate of such missing energy events depends strongly on the mass of the colored particle that is produced and only weakly on other properties of the model. So it is reasonable to estimate the discovery potential of the LHC by looking at the predictions for the special case of supersymmetry. In Fig. 1, we show the estimates of the ATLAS collaboration for the discovery of missing energy events at various levels of the LHC integrated luminosity [25]. For the purpose of this discussion, it suffices to follow the contours of mass for the squarks and gluinos that are the primary colored particles produced. According to the figure, if either the squark or the gluino has a mass below $1000 \mathrm{GeV}$, the missing energy events can be discovered with an integrated luminosity of $100 \mathrm{pb}^{-1}$, about $1 \%$ of the LHC first-year design luminosity. Thus, we will know very early in the LHC program that the LHC is producing a WIMP candidate. This will open the way to detailed studies of the role of this WIMP in astrophysics.

\subsection{Qualitative determination of WIMP parameters}

For reasons that we will detail in the next section, it is very important after the discovery of the WIMP to identify it qualitatively, that is, to single out what theory gives rise to this particle and what its basic interactions are. This next step may turn out to be very difficult at the LHC.

The reason for this is just the converse of the argument that the characteristic signature of the WIMP is observed missing momentum. At a proton collider such as the LHC, reactions that produce heavy particles are initiated by quarks and gluons inside the proton. We do not know a priori how much of the momentum of the proton each initial particle carries. Since we do not observe the final-state WIMPs, we also cannot learn the energies and momenta of the produced particles from the final state. If we cannot find the rest frame of the massive particles, it is very difficult to determine the spins of these particles or to specifically identify their decay modes.

As a concrete illustration of this argument, consider the four models of the decay of a colored primary particle shown in Fig. 2. Examples (a) and (b) are drawn from models of supersymmetry in which the WIMP is the supersymmetric partner of the photon or neutrino. Examples (c) and (d) are drawn from models of extra dimensions in which the WIMP is, similarly, a higher-dimensional excitation of a photon or a neutrino. The observed particles in all four decays are the same; the subtle differences in their momentum distributions are obscured by the uncertainty in reconstructing the frame of the primary colored particle. It is possible to make use of more model-dependent features. In the recent papers [26-28] specific features of the models have been identified that can distinguish the cases of supersymmetry and

extra dimensions. Still, it is likely that, from the LHC experiments alone we will be left with several competing possibilities for the qualitative identity of the WIMP. 
a.)

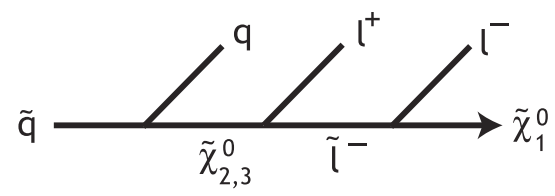

b.)

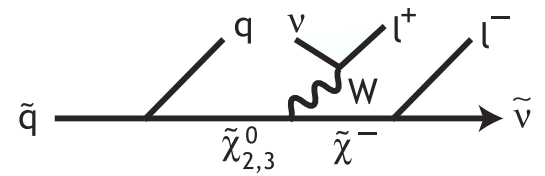

c.)

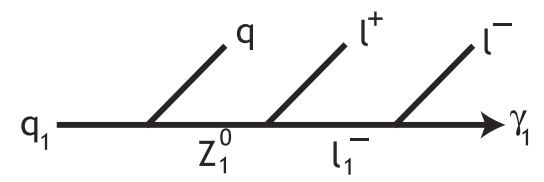

d.)

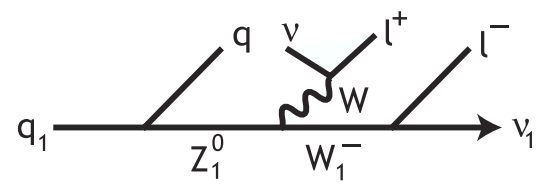

Figure 2: Four scenarios for decay chains observed at LHC. Each exhibits jets, hard leptons, and missing energy. Distinguishing between these cases by detailed study of energy distributions may not be possible with LHC alone.

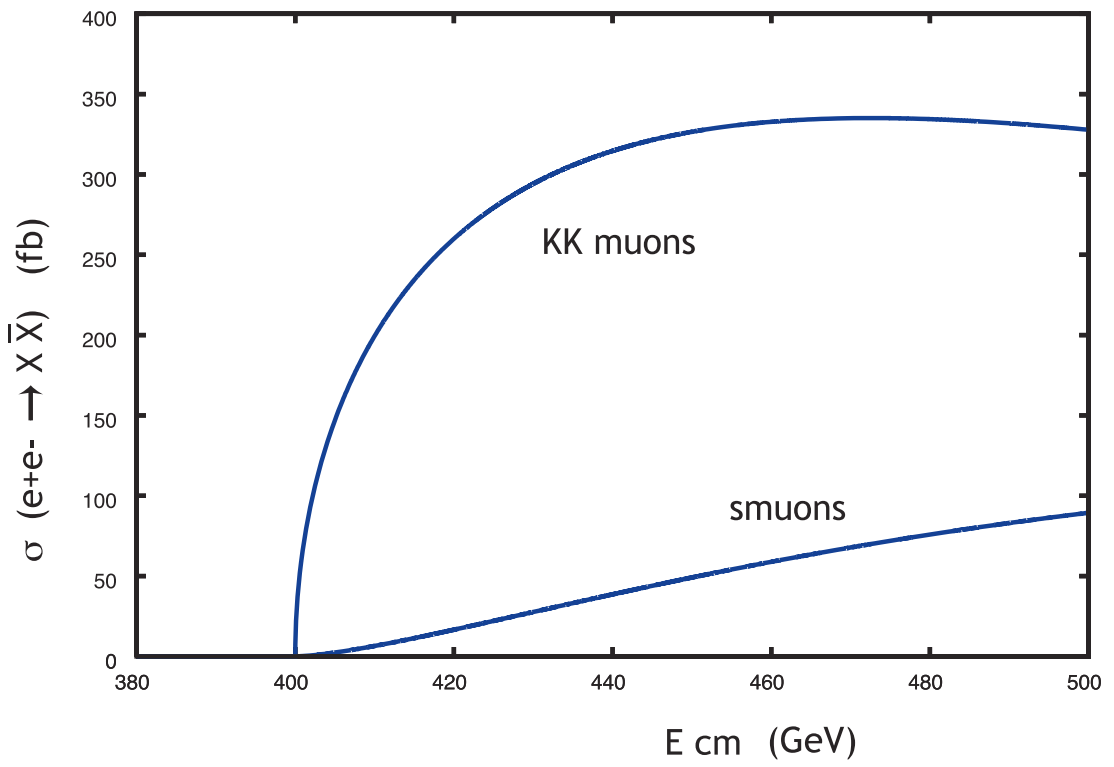

Figure 3: Threshold behavior of pair production cross sections for spin 1/2 (KK muon) and spin 0 (smuon) counterpart to the Standard Model muon. These distributions are easily distinguished by an $e^{+} e^{-}$collider. 
Fortunately, another tool is likely to be available to particle physicists. At an electron-positron collider, the pair production process $e^{+} e^{-} \rightarrow X \bar{X}$ is gives an exquisite diagnostic of the quantum numbers of a massive particle $X$. As long as only the diagrams with annihilation through $\gamma$ and $Z$ are relevant, the angular distribution and threshold shape of the reaction are characteristic for each spin, and the normalization of the cross section directly determines the $S U(2) \times U(1)$ quantum numbers. These tests can be applied to any particles with electric or weak charge whose pair-production thresholds lie in the range of the collider. In Figure 3, we show one example of such a test for models (a) and (c) of Fig 2, by plotting the threshold behavior of the pair-production cross sections for the supersymmetry or extra-dimensional muon partner that appears at the last stage of the decay process. This single measurement would already pin down the spin and quantum numbers of the particle and bring us a long way toward the qualitative identification of the model. Particle physicists are now designing an electron-positron collider, the International Linear Collider (ILC), which will reach $500 \mathrm{GeV}$ in the center of mass in its initial stage and will be upgradable in energy to about $1000 \mathrm{GeV}$.

The discussion of this and the previous section highlights the contrasting strengths of the LHC and the ILC, and of the technologies of proton and electron colliders. The LHC can more easily reach high energies and offers very large cross sections for specific states of a model of new physics. The ILC typically reaches fewer states in the new particle spectrum, but it gives extremely incisive measurements of the properties of the particles that are available to it. Also, as we will see, these particles are typically the ones on which the dark matter density depends most strongly. Both LHC and ILC can make precision measurements, but the measurements at the ILC typically have a more direct and model-independent interpretation. In our discussion in Section 4-7, we will see many examples in which the greater energy reach of the LHC contrasts with the greater specificity of the measurements from the ILC.

\subsection{Quantitative determination of WIMP parameters}

For the purpose of understanding dark matter, what we actually want from an understanding of the WIMP in particle physics is the ability to predict the WIMP's relic density and detection cross sections. Given that the WIMP is not observable in the high-energy physics experiments, it is not so obvious how to make these predictions, or, even, that the predictions can be made. The only strategy available to us is to understand the underlying particle physics model well enough to fix the interactions of the WIMP. To do this, we must determine its couplings and the masses and properties of the observable particles to which it couples.

As a matter of principle, this is a very difficult undertaking. The one advantage that we have is that the cross sections of the WIMP that are the most important in 
astrophysics involve very low energies. The relic density is determined by the WIMP annihilation cross section at temperatures such that $T / m_{\chi} \sim 1 / 25$, corresponding to nonrelativistic motion [13]. When we observe the WIMP through its annihilation processes, the annihilation energy is very close to threshold. In direct detection of WIMPs, or in the capture of WIMPs into the earth or the sun, the WIMPs are moving with a velocity $v / c \sim 10^{-3}$. Though we will see some exceptions to this, it is typical that the most important diagrams for computing these cross sections involve the lightest particles in the model. If we can characterize these particles and measure their properties with precision, we can reach the goal of making microscopic predictions of the WIMP properties.

We do not know a way to give a general proof of this claim, but we can illustrate its validity through studies of models. In Section 3, we will explain in detail how we will test this claim for supersymmetry models with neutralino dark matter.

\subsection{Astrophysical dark matter measurements: relic density}

In the next three sections, we will review the astrophysical measurements that will require cross sections and other particle properties that might be determined by particle physics measurements. The first of these is the most basic property of dark matter, its cosmic mass density.

The mass density of dark matter is already known today to impressive accuracy, and this accuracy is expected to improve significantly before the end of the decade. The analysis of fluctuations in the cosmic microwave background (CMB) - in particular, the measurement of the acoustic peaks that reflect oscillations in the plasma that filled the universe at temperatures just below $1 \mathrm{eV}$-allow determinations of the density of baryonic and non-baryonic matter. The current value of the dark matter density, dominated by the data from the WMAP experiment [6], is [1]

$$
\Omega_{\chi} h^{2}=0.111 \pm 0.006
$$

This is already a determination to $6 \%$ accuracy. In 2007, we expect the launch of the Planck satellite, which will give an even more precise measurement of the properties of the CMB. From this experiment, we can expect an improvement in the accuracy of $\Omega_{\chi} h^{2}$ to $0.4 \%$ [7].

It will be very difficult for microscopic predictions of the WIMP density to match this level of precision. But we will see that, in the specific models that we will consider, it is possible to give a microscopic prediction of the WIMP density to an accuracy of $20 \%$ or better. Thus, it will be a quite nontrivial test to compare the microscopic prediction to the density determined from the CMB. 
A discrepancy between the microscopic and CMB values could arise for many reasons. The WIMP could provide only a portion of the dark matter, with other portions arising from different types of particles. The WIMP could decay to a lighter and even more weakly interacting particle, a 'super-WIMP' [29]. In this case, experiments on astrophysical WIMP detection should see nothing, but particle physics experiments might find evidence for the WIMP instability both from the new particle spectrum and from direct observation of the decay [30,31]. The density of WIMPs could be diluted between the temperature of WIMP decoupling $(T \sim \mathrm{GeV})$ and the temperature of primordial nucleosynthesis by some mechanism of entropy production, due to a phase transition or late particle decay. On the other side, the WIMP density could mainly be generated out of equilibrium, during reheating to $\mathrm{TeV}$ temperatures or from the decay of heavy particles to WIMPs. In supersymmetry models, this scenario has been studied in models of anomaly-mediated supersymmetry breaking [32,33]. These models contain large annihilation cross sections and so predict large astrophysical signals of WIMP annihilation.

In the study of primordial nucleosynthesis, both late entropy production and nonthermal processes have been considered, along with more exotic effects from new physics. But, in fact, the predictions of primordial nucleosynthesis are in remarkable agreement with the predictions based on standard cosmology combined with detailed laboratory measurements of low-energy nuclear cross sections [34]. This gives us confidence that our cosmological model is correct back to times of the order of 1 minute after the Big Bang. From this experience, we conclude that it is possible also that the measured and predicted WIMP density might turn out to be in excellent agreement, verifying standard cosmology back to times of $10^{-9}$ seconds.

If the CMB and microscopic determinations of the WIMP density within their individual accuracies, it will be tempting to impose the more stringent astrophysical constraint on the particle physics model. At present, we do not know the particle physics model, and imposing the constraint (3) does not seem to exclude any qualitative possibilities, though it does narrow the parameter space if a given model is assumed. After the particle physics model is known, the stringent constraint on the dark matter density from Planck can have more interesting consequences, as we will see in our examples.

\subsection{Astrophysical dark matter measurements: direct detection}

If the microscopic cross sections measured at colliders agree with the CMB measurements of the dark matter density, a crucial uncertainty remains, alluded to in the previous section. Does the dark matter candidate in addition have a lifetime longer than the age of the universe, or is the consistency merely a coincidence? A complete

picture of dark matter requires that the candidate particle be observed as the major 
constituent of our galaxy. This can be accomplished by direct or indirect detection, discussed in this section and the next.

Direct detection of WIMPs in sensitive low-background experiments involves the cross sections for WIMP scattering from nucleons near threshold. The expressions for these cross sections naturally divide into spin-dependent and spin-independent isoscalar and isovector contributions. The spin-independent isoscalar term is enhanced in WIMP-nucleus cross sections by factors of $A^{2}$, so we will emphasize the prediction of this contribution. The predictions for direct detection cross sections in supersymmetry models are typically displayed on scatter plots that cover five orders of magnitude [2]. As we will see, data from the LHC and the ILC can significantly narrow this range.

Predictions for the rate in direct detection experiments rely on the assumption that the average density of dark mater in the disk of the galaxy can be used to estimate the flux of dark matter that impinges on a detector on the earth. This density is uncertain, but, in addition, we do not expect the density of dark matter to be constant over the disk. In models of cold dark matter, the galaxy is assembled from smaller clusters of dark matter. The initial situation is inhomogeneous, and these inhomogeneities are not expected to be smoothed out in the time since the galaxy was formed.

The local halo density is inferred by fitting to models of the galactic halo. These models are constrained by a variety of observations, including the rotational speed at the solar circle and other locations, the total projected mass density (estimated by considering the motion of stars perpendicular to the galactic disk), peak to trough variations in the rotation curve ('flatness constraint'), and microlensing. Gates, Gyuk, and Turner [35] have collected these constraints and estimated the local halo density to lie between $4 \times 10^{-25} \mathrm{~g} / \mathrm{cm}^{-3}$ and $13 \times 10^{-25} \mathrm{~g} / \mathrm{cm}^{-3}$. Limits on the density of MACHO microlensing objects imply that at least $80 \%$ of this is cold dark matter. The velocity of the WIMPs would be close to the galactic rotation velocity, $230 \pm 20$ $\mathrm{km} / \mathrm{sec}[36]$.

These constraints rely on the assumption that the dark matter has a smooth distribution in the galactic halo. There is much evidence, coming primarily from high resolution N-body simulations, to suggest that the dark matter distribution is in fact highly irregular. Using a hierarchical clustering model of galactic structure, Stiff, Widrow, and Frieman have argued that the solar neighborhood might be expected to be located within a clump of dark matter with only slightly higher local density but a velocity distribution peaked at a relatively large value [37]. Other authors have argued for larger density variation in the galactic distribution of dark matter. Sikivie and Ipser [38] have proposed that spherical infall of dark matter on to developing galaxies will tend to accumulate along singular surfaces or caustics, leading to very 
large local fluctuations. An even more extreme model was recently put forward by Diemand, Moore, and Stadel [39], who argued that WIMPs are likely to appear in clusters of mass comparable to the mass of the earth and densities roughly $10^{3}$ larger than the average density of the disk. In addition to these models that rely on the general features of galaxy formation, the local geography of our region of the galaxy might affect the dark matter density. For example, Freese, Gondolo and Newberg have proposed that the Sagittarius stream should add up to $23 \%$ to the local dark matter density, with a characteristic annual modulation [40]. These models illustrate not only that there is a large uncertainty in the value of the local dark matter flux at the earth but also that the understanding of this value relative to the overall average density of dark matter is an interesting astrophysical question.

If we view these questions as uncertainties, we must say that direct detection experiments cannot by themselves put constraints on the microscopic scattering cross sections of WIMPs. On the other hand, if we could obtain the microscopic cross sections from particle physics, the event rate in direct detection experiments would directly measure the local flux of WIMP dark matter. If direct detection experiments failed to detect dark matter, it would be even more important to have the microscopic cross section in order to establish strong upper bounds on the local WIMP density.

Parenthetically, we would like to call attention to an aspect of direct detection experiments that is very important but is not often emphasized. It is very likely that direct detection experiments will see evidence of WIMPs in the same time frame, before the end of the decade, that the LHC observes missing energy events. In this case, it will be essential to compare the mass of the WIMP observed in each setting. In supersymmetry models, as we will discuss in Sections 4-7, the kinematics of events with squark production and decay can determine the mass of the WIMP to about $10 \%$ accuracy. We believe that this type of analysis will give the WIMP mass to similar accuracy in typical models of the general class discussed in Section 2.2.

Direct detection experiments can determine the mass of the WIMP by measuring the recoil energy $E_{R}$. This varies with the mass of the WIMP, with a resonance where the WIMP mass equals the target mass. Roughly, one expects

$$
\left\langle E_{R}\right\rangle \approx \frac{2 v^{2} m_{T}}{\left(1+m_{T} / m_{\chi}\right)^{2}},
$$

where $m_{T}$ is the target mass and $v$ is the WIMP velocity, with corrections depending on the precise target material and the properties of the detector [41]. Assuming the standard velocity distribution in smooth halo models, with the $10 \%$ uncertainty quoted above, an experiment with a Xenon or Germanium target that detects 100 signal events for a WIMP of mass $m_{\chi}=100 \mathrm{GeV}$ can expect to measure the mass of this particle to 20-25\%. A discrepancy between the value of the WIMP mass observed 
in direct detection and that found at the LHC could signal a nonstandard velocity distribution. At a later stage, this could be checked by comparing the detection rate, which is proportional to the flux of WIMPs, to the cross section determined from high-energy collider data.

In Fig. 4, we show a comparison of the determination expected for the WIMP mass from the LHC data and from the analysis of data from the SuperCDMS detector $[42,43]$ for one of the supersymmetry models that we introduce in Section 3.2. The contours are based on a sample of 27 events and so are statistically limited. Still, it is clear that a nontrivial comparison of WIMP masses between accelerator and astrophysical experiments will be possible.

Since the annual modulation of the WIMP velocity relative to earth is very well known, the measurement of the annual variation of the WIMP detection rate and the recoil energy spectrum provides a method for a more accurate and model-independent determination of the WIMP [45]. A strategy for a very high-accuracy measurement has been described by Bourjaily and Kane [46]. However, this would require a sample of direct detection events about 100 times larger than those in the illustrative examples we will present here.

Finally, we comment on the possibility that WIMPs measured at colliders make up only a fraction of the dark matter. In this case, the annihilation cross sections tend to be larger than the expected $1 \mathrm{pb}$, and also the direct detection cross sections tend to be large. In fact, these effects broadly speaking cancel: a WIMP making up $10 \%$ of the cosmic dark matter tends to have cross sections 10 times as large as expected, thus the direct detection rate is roughly independent of the inferred relic density [47].

\subsection{Astrophysical dark matter measurements: WIMP annihilation}

WIMP annihilation could potentially be observed through gamma ray, positron, antiproton, antideuteron, and neutrino signals. Of these, the observations through gamma rays is the simplest and most robust. There are already claims that an excess of gamma rays from the galactic center gives evidence for WIMP dark matter [48]. We will examine how this study will be aided by collider physics determinations of the WIMP annihilation cross section.

The flux of gamma rays observed on earth from WIMP annihilation is given by the formula

$$
E_{\gamma} \frac{d \Phi_{\gamma}}{d E_{\gamma} d \Omega}=\frac{1}{2}\left(\sigma_{\chi \chi} v\right) \cdot \frac{E_{\gamma}}{\sigma_{\chi \chi}} \frac{d \sigma_{\chi \chi}}{d E_{\gamma}} \cdot \frac{1}{4 \pi m_{\chi}^{2}} \cdot \int d z \rho^{2}(z),
$$

where $\sigma_{\chi \chi} / 2$ is the annihilation cross section near threshold, (which typically behaves 


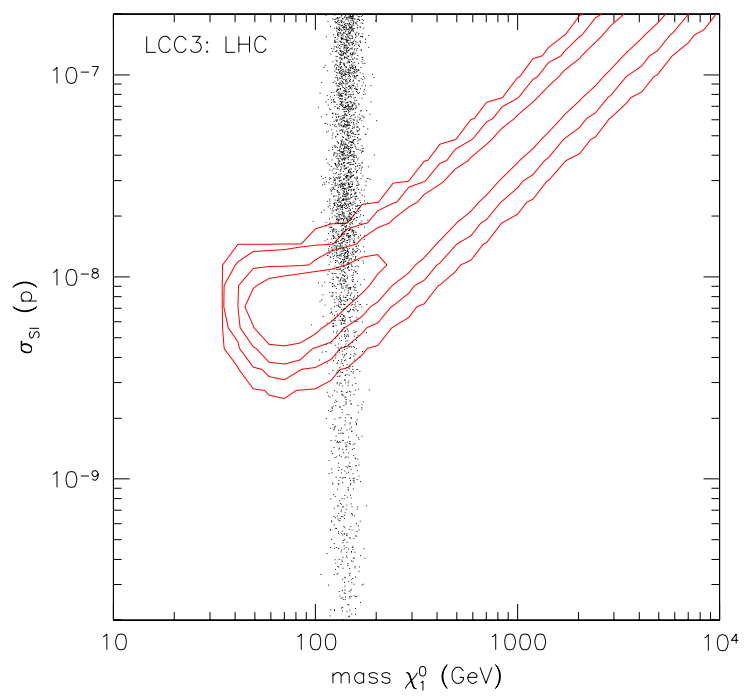

Figure 4: Projected significance contours $(1,2,3,4 \sigma)$ in the plane of WIMP mass versus cross section for an observation of dark matter by the SuperCDMS experiment $(25 \mathrm{~kg}$ target, two year dataset) $[42,44]$, compared to the determination of these parameters from data from the LHC. The projections are done using the model LCC3, to be defined in Section 3.2.

as $1 / v), z$ is a coordinate along the line of sight, and $\rho$ is the WIMP mass density. The factor of $1 / 2$ accounts for the fact that the incoming particles are identical. The first three factors come from microscopic physics; the final factor is a question of astrophysics. The density integral is commonly written

$$
\int d z \rho^{2}(z)=r_{0} \rho_{0}^{2} J(\Omega)
$$

where $r_{0}=8.5 \mathrm{kpc}$ is the distance to the center of the galaxy and $\rho_{0}=0.3 \mathrm{GeV} \mathrm{cm}^{-3}$ $\left(5.34 \times 10^{-25} \mathrm{~g} / \mathrm{cm}^{3}\right)$ is a reference value of the local density of dark matter. The quantity $J(\Omega)$ is then dimensionless.

It is likely that the microscopic quantities that appear in (5) could be estimated to an accuracy of $20 \%$ even in the early stages of the study of WIMPs in particle physics experiments. The mass $m_{\chi}$ will be determined to better than $10 \%$ accuracy from the kinematics of missing energy events at the LHC. We will show in Section 8.1 that the second factor in (5), the shape of the gamma-ray spectrum, is almost completely model-independent and can be obtained accurately from particle physics simulations. For the first factor, the total annihilation cross section, it is tempting to insert the value (2) from the relic density. This is sometimes, but not always, a good approximation, depending on the qualitative properties of the supersymmetry 
spectrum. We will discuss the systematics of this quantity in Section 8.1. In many physics scenarios, all three quantities will be known well enough already from the LHC data to quantitatively interpret gamma ray observations.

It is very important that the particle physics factors in (5) can be determined from microscopic measurements, because the last factor raises major questions about the structure of the dark matter halo of the galaxy. Let us first discuss the dark matter profile at the galactic center. A typical profile of the smoothed dark matter halo is written

$$
\rho(r)=\rho_{0}\left(r / r_{0}\right)^{\gamma}\left(\frac{1+\left(r_{0} / a\right)^{\alpha}}{1+(r / a)^{\alpha}}\right)^{(\beta-\gamma) / \alpha},
$$

where $\alpha, \beta, \gamma$ are parameters and the core size $a$ should be about $1 \mathrm{kpc}$. A canonical choice justified by numerical simulations of galaxy formation by cold dark matter is the Navarro-Frenk-White profile: $(\alpha, \beta, \gamma)=(1,3,1)$ [49]. However, this choice is controversial. Moore et al. have argued for a much steeper profile at the galactic center: $(\alpha, \beta, \gamma)=(1.5,3,1.5)[50]$. For this profile, the integral over $\rho^{2}(z)$ formally diverges at the galactic center, though the divergence is only logarithmic and can be cut off, for example, at the Schwarzschild radius of the black hole at the center of galaxy. The simulations that we are discussing include only dark matter, not baryonic matter. It has been argued that the dissipative effects of baryonic matter smooth this distribution, perhaps to the 'isothermal' profile $(\alpha, \beta, \gamma)=(2,2,0)$. The variation in (5) near the galactic center for several representative profiles is shown in Fig. 5. We present both the profile functions themselves and the value of $J(\Omega)$ averaged over a disk of varying solid angle centered on the galactic center. The predictions for $\langle J(\Omega)\rangle$ span six orders of magnitude between models. Further, these estimates are all smooth profiles, and we have already seen that the distribution of dark matter might be clumpy. Such small-scale structure would enhance indirect detection rates by the ratio

$$
B=\left\langle\rho^{2}\right\rangle /\langle\rho\rangle^{2},
$$

sometimes called the 'boost factor'. Here again, we see that dark matter detection rates depend on distributions that are highly uncertain and touch on major questions of astrophysics. It would be interesting to extract these distributions from the experiments, or even to obtain upper limits on them. For this, we need to know the microscopic cross sections from particle physics.

This conclusion applies also to other possible sources of gamma rays from WIMP annihilation. For gamma rays from the centers of local group and other nearby galaxies, the considerations are very similar to those for the galactic center [52]. Simulations of galaxy formation with cold dark matter predict many more dwarf companions of the Milky Way than are actually observed. Probably, many of these have been tidally disrupted or absorbed. However, it is likely that some of these 

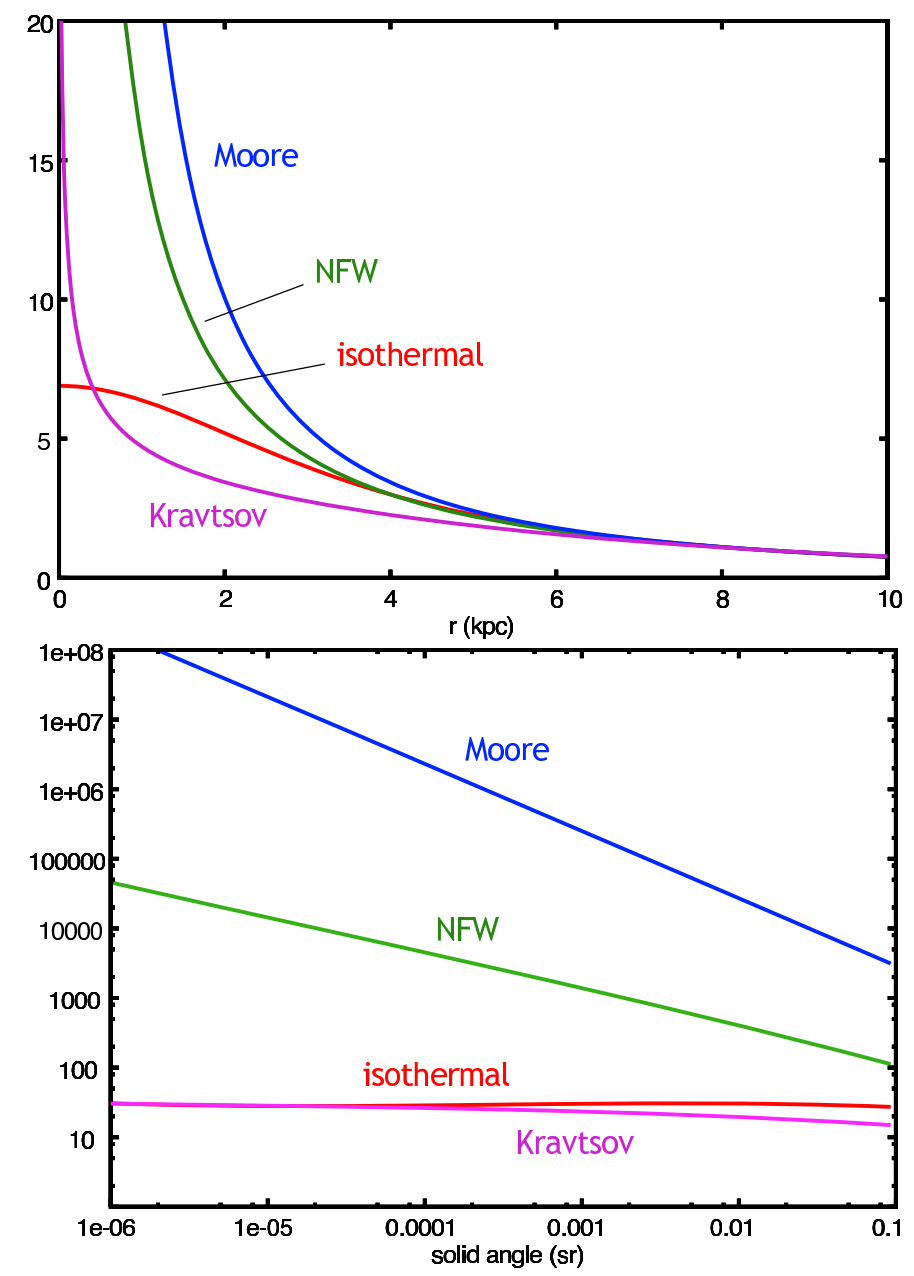

Figure 5: (a) Suggested profiles of the dark matter mass density near the galactic center [49-51]. (b) Corresponding values of $\langle J\rangle$ at the galactic center, as a function of the angular resolution indicated as a circle size in sr. 
unobserved dwarf galaxy are actually present as pure dark matter halos from which the baryonic gas has been blown out [53]. The GLAST telescope, with its $\pi$ angular coverage for gamma rays, has the ability to search for these objects. Even upper limits are interesting, but their interpretation will depend critically on knowledge of the microscopic factors in (5).

The expectations for observation of positrons, neutrinos, antiprotons and antideuterons from WIMP annihilation depend on more specific details of the physics model. We will discuss the subject of positron signals further in Section 8.2.

\subsection{Summary: steps toward an understanding of WIMP dark matter}

The program of experiments that we have described in this section has the potential to give us a complete understanding of the nature of WIMP dark matter. The steps are the following:

1. Discover missing-energy events at a collider and estimate the mass of the WIMP.

2. Observe dark matter particles in direct detection experiments and determine whether their mass is the same as that observed in collider experiments.

3. Determine the qualitative physics model that leads to missing-energy events

4. Determine the parameters of this model that predict the relic density.

5. Determine the parameters of this model that predict the direct and indirect detection cross sections

6. Measure products of cross sections and densities from astrophysical observations to reconstruct the density distribution of dark matter.

If dark matter is composed of a single type of WIMP, this program of measurements should reveal what this particle is and how it is distributed in the galaxy. If the composition of dark matter is more complex, we will only learn that by carrying out this program and finding that it does not sum to a complete picture. Hopefully, further evidence from the microscopic theory will suggest other necessary ingredients.

The main goal of the remainder of this paper is to show that the experiments foreseen for the LHC and ILC will be able to predict the microscopic dark matter cross sections with sufficient accuracy that we can carry out this program. In the next section, we will describe our strategy for addressing this question. 


\section{Models of neutralino dark matter}

As we have already noted in Section 2.3, the annihilation and detection cross sections needed to interpret observations of WIMP dark matter cannot be measured directly in high-energy physics experiments. To predict these cross sections, we must interpret experimental data on the spectra and parameters of the underlying physics model. To do this, we must understand, at a qualitative level, what the correct model is. We must then convert measurements of the spectrum of new particles into constraints on the underlying model parameters. Some care should be taken in the choice of the model. If we work in too restrictive a model context, this procedure will artificially restrict the solutions, and we will claim an unjustified small accuracy for our predictions. Thus, to evaluate how accurately collider data will predict the dark matter cross section, we need to work within a model that, under overall restrictions from spin and quantum number measurements, has a large parameter space and allows a wide variety of physical effects to come into play.

Among models of physics beyond the Standard Model, the only one in which dark matter properties have been studied over such a large parameter space is supersymmetry [54]. The Minimal Supersymmetric Standard Model (MSSM) introduces a very large number of new parameters and allows many physically distinct possibilities for the mass spectrum of new particles. Thus, our strategy for evaluating the implications of collider data for dark matter cross sections will be to study a set of MSSM parameter points which illustrate the variety of physics scenario that this general model can contain. In each case, we will systematically scan the parameter space of the MSSM for models that are consistent with the expected collider measurements. We hope that the insights obtained from this study will lead us to conclusions of broader applicability about the power to high-energy physics measurements to restrict the properties of dark matter.

\subsection{Mechanisms of neutralino annihilation}

From here on, then, we restrict our attention to models with supersymmetry in which the role of the WIMP $\chi$ is taken by the lightest 'neutralino' - a mixture of the superpartners of $\gamma$ and $Z$ ('gauginos') and the superpartners of the neutral Higgs bosons ('Higgsinos'). Depending on the spectrum and couplings of the superpartners, several different reactions can dominate the process of neutralino pair annihilation. Some of the most important possibilities are illustrated in Fig. 6.

The simplest possibility (Fig. 6(a)) is that neutralinos annihilate to Standard Model fermions by exchanging their scalar superpartners. Sleptons are typically lighter than squarks, so the dominant reactions are $\chi \chi \rightarrow \ell^{+} \ell^{-}$. It turns out, however, 
a.)

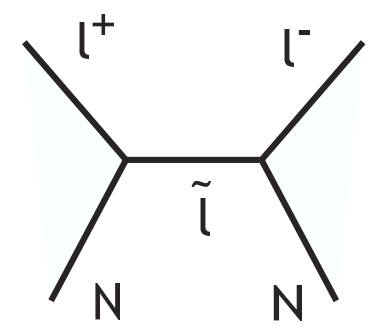

c.)

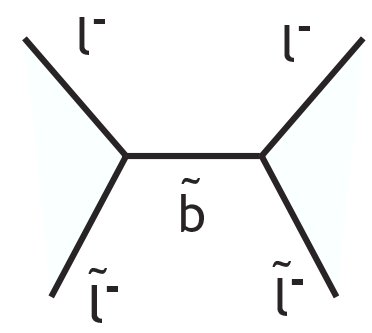

b.)

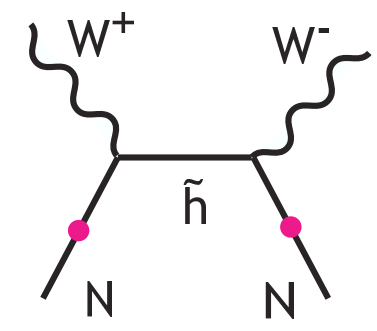

d.)

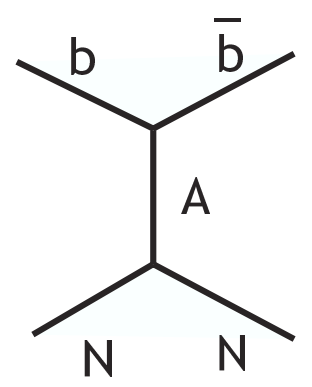

Figure 6: Four neutralino annihilation reactions that are important in different regions of the MSSM parameter space: (a) annihilation to leptons, (b) annihilation to $W^{+} W^{-}$, (c) coannihilation with $\widetilde{\tau},(\mathrm{d})$ annihilation through the $A^{0}$ resonance.

that this reaction is less important than one might expect over most of the supersymmetry parameter space. Because neutralinos are Majorana particles, they annihilate in the S-wave only in a configuration of total spin 0. However, light fermions are naturally produced in a spin-1 configuration, and the spin-0 state is helicity-suppressed by a factor $\left(m_{\ell} / m_{\chi}\right)^{2}$. The dominant annihilation is then in the P-wave. Since the relic density is determined at a temperature for which the neutralinos are nonrelativistic, the annihilation cross section is suppressed and the prediction for the relic density is, typically, too large. To obtain values for the relic density that agree with the WMAP determination, we need light sleptons, with masses below $200 \mathrm{GeV}$.

Neutralinos can also annihilate to Standard Model vector bosons. A pure $U(1)$ gaugino ('bino') cannot annihilate to $W^{+} W^{-}$or $Z^{0} Z^{0}$. However, these annihilation channels open up if the gaugino contains an admixture of $S U(2)$ gaugino ('wino') or Higgsino content (Fig. 6(b)). The annihilation cross sections to vector bosons are large, so only a relatively small mixing is needed.

The annihilation to third-generation fermions can be enhanced by a resonance close to threshold. In particular, if mass of the CP odd Higgs boson $A^{0}$ is close to $2 m_{\chi}$, the resonance produced by this particle can enhance the S-wave amplitude for neutralino annihilation to $b \bar{b}$ and $\tau^{+} \tau^{-}$(Fig. 6(d)). 
If other superparticles are close in mass to the neutralino, these particles can have significant densities when the neutralinos decouple, and their annihilation cross sections can also contribute to the determination of the relic density through a coannihilation process. If the sleptons are only slightly heavier than the neutralino, the reactions $\widetilde{\ell} \chi \rightarrow \gamma \ell$ and $\widetilde{\ell} \rightarrow \ell \ell$ can proceed in the S-wave and dominate the annihilation (Fig. 6(c)). Coannihilation with $W^{+}$partners ('charginos') and with top squarks can also be important in some regions of the MSSM parameter space.

A common feature of all four mechanisms is that the annihilation cross section depends strongly both on the masses of the lightest supersymmetric particles and on the mixing angles that relate the original gaugino and Higgsino states to the neutralino mass eigenstates. Both sets of parameters must be fixed in order to obtain a precise prediction for the relic density.

A supersymmetry model that produces a relic density of neutralinos in the range required by the WMAP data should implement one of these mechanisms. To the extent that the operation of the mechanism requires special conditions on the supersymmetry spectrum, the neutralino relic density will depend more sensitively on the spectrum parameters than we might at first have estimated. We will see the effects of this observation in our model studies.

\subsection{Choice of benchmark models}

We would now like to choose four specific supersymmetry models that illustrate the four mechanisms described in the previous section.

Let us first discuss the parameters of the MSSM. The most general formulation of the MSSM has 108 parameters beyond those of the Standard Model. However, many of these parameters violate $\mathrm{CP}$ or induce flavor-changing neutral current processes and are thus tightly constrained. If we include only interactions that conserve $\mathrm{CP}$ and flavor, we find the following set of parameters:

- gaugino and Higgsino masses: $m_{1}, m_{2}, m_{3}, \mu$

- slepton masses: $m^{2}\left(L_{i}\right), m^{2}\left(\bar{e}_{i}\right), i=1,2,3$

- squark masses: $m^{2}\left(Q_{i}\right), m^{2}\left(\bar{u}_{i}\right), m^{2}\left(\bar{d}_{i}\right), i=1,2,3$

- Higgs potential terms: $m_{A}, \tan \beta$

- $A$ terms: $A_{\tau}, A_{b}, A_{t}$

a total of 24 new physics parameters. 


\begin{tabular}{l|cccccc|c||c} 
Point & $m_{0}$ & $m_{\frac{1}{2}}$ & $\tan \beta$ & $A_{0}$ & $\operatorname{sign} m u$ & $m_{t}$ & reference & $\Omega_{\chi} h^{2}$ \\
\hline LCC1 & 100 & 250 & 10 & -100 & + & 175 & {$[63]$} & 0.192 \\
LCC2 & 3280 & 300 & 10 & 0 & + & 175 & {$[64]$} & 0.109 \\
LCC3 & 213 & 360 & 40 & 0 & + & 175 & {$[65]$} & 0.101 \\
LCC4 & 380 & 420 & 53 & 0 & + & 178 & {$[67]$} & 0.114 \\
\hline SPS1a $^{\prime}$ & 70 & 250 & 10 & -300 & + & 175 & {$[68]$} & 0.115
\end{tabular}

Table 1: mSUGRA parameter sets for four illustrative models of neutralino dark matter. Masses are given in GeV. The table also lists the value of $\Omega_{\chi} h^{2}$. The references given are the primary references for simulation studies of the accuracy of spectrum measurements at colliders. The point SPS1a' has a phenomenology similar to that of LCC1 sub gives a more correct value of the relic density.

Most papers on neutralino dark matter adopt additional, ad hoc, assumptions to reduce this parameter list to a smaller set. Most typically, they assume unification of the various mass terms at the grand unification scale. This assumption, called 'mSUGRA' or 'cMSSM', reduces the parameter set to four, plus a choice of sign: $m_{0}$, $m_{1 / 2}, A_{0}, \tan \beta, \operatorname{sign}(\mu)$. The restricted parameter space of mSUGRA is very interesting to illustrate the various possibilities for neutralino dark matter. In particular, this subspace contains examples of all four mechanisms that we have presented in the previous section [57-59].

This makes it very convenient to choose parameter points from the mSUGRA subspace. In Table 1, we list the four parameter sets that define the supersymmetry models that we will study in detail [60]. The spectra for these points, and for the more general supersymmetry parameter points that we will study, are computed with ISAJET 7.69 [8]. Results for the relic density and for neutralino detection cross sections are computed with DarkSUSY-4.1 [9]. We have checked that Micromegas 1.3 [61] gives similar results for the relic density.

The four points listed in Table 1 illustrate the four scenarios described in the previous section. Point LCC1 is identical to the point SPS1a [62] whose collider phenomenology is studied in some detail in [63]. This point has light sleptons, with neutralino annihilation dominated by roughly equal annihilation cross sections to $e^{+} e^{-}, \mu^{+} \mu^{-}$, and $\tau^{+} \tau^{-}$. The sleptons are not quite light enough; the spectrum achieves a relic density $\Omega h^{2}=0.19$, almost doubly the WMAP value. Point LCC2 is chosen as a point with substantial gaugino-Higgsino mixing at which the neutralino annihilation is dominated by annihilation to $W^{+} W^{-}, Z^{0} Z^{0}$, and $Z^{0} h^{0}$. Point LCC3 is chosen in the region where coannihilation with the $\widetilde{\tau}$ plays an important role. Point LCC4 is chosen in a region where the $A^{0}$ resonance makes an important contribution to the neutralino annihilation cross section.

The four points are intentionally chosen so that the lightest particles of the super- 
symmetry spectrum can be observed at the ILC at its initial center of mass energy of $500 \mathrm{GeV}$. This is also the most probable region of the parameter space, since in this region the dynamics of supersymmetry can generate electroweak symmetry breaking without extensive fine-tuning of parameters [69].

It will be important to us to have well-justified estimates for the accuracy with which high-energy physics experiments can measure the supersymmetry spectrum parameter at these points. For these reason, we have chosen to analyze points in the MSSM parameter space at which simulations have been carried out to estimate the ability of colliders to measure parameters of the supersymmetry spectrum. For the point LCC1 or SPS1a, an extensive set of simulation studies for both LHC and ILC is described in [63]. These studies assume a luminosity sample of $300 \mathrm{fb}^{-1}$ and LHC and $500 \mathrm{fb}^{-1}$ for ILC. They include realistic modeling of particle detection and Standard Model backgrounds. For the other LCC points, similarly detailed studies have been performed only for the ILC [70]. However, as we will see, the conclusions of [63] and other LHC studies such as those reported in [71] can be used to estimate the LHC capabilities at these points. The point LCC2 has been studied for the ILC by Alexander, et al., in [64]. The point LCC3 has been studied by Khotilovich, et al., in $[65,66]$. The analysis of this point requires a precision measurement of the $\widetilde{\tau}$ mass, and the question of how well that can be done has also been studied at related point by Bambade, et al. in [72]. The point LCC4 has been studied in the ILC environment in $[67]$.

The reader will note that the point LCC1 gives a value of $\Omega_{\chi} h^{2}$ outside the range preferred by the current data. We are not troubled by this, except to note that the appropriate figure of merit is the relative accuracy, rather than the absolute accuracy, with which $\Omega_{\chi} h^{2}$ can be determined. Other authors, however, have been concerned about this and have extrapolated the simulation results obtained at LCC1 (SPS1a) to a nearby point SPS1a' which, because it pushes into the stau coannihilation region, predicts a relic density $\Omega_{\chi} h^{2}=0.115$ [68]. In this paper, we have done our main analysis at the original point in order to cleanly separate examples in which different physics determines the relic density. However, Nojiri, Polesello, and Tovey [11] have performed a detailed analysis of the LHC prediction of the relic density at SPS1a', and so, for comparison, we will also present the corresponding results for this point in our framework.

The four points are chosen within the limited subspace of mSUGRA models. However, when we interpret measurements from the colliders, we will not want to assume that the true supersymmetry model lives in this subspace. There is no compelling physics argument for the mSUGRA assumptions. More importantly, the restriction to a four-parameter model induces correlations between parameters of the supersymmetry spectrum, for example, $\tan \beta, \mu$, and the top squark mass, that should properly 
be considered independent. Some interesting studies have been performed in which the capability of the LHC to predict $\Omega_{\chi} h^{2}$ has been assessed by scanning over the parameter space of mSUGRA [73,74]. However, we believe that that this restriction makes the conclusions excessively optimistic.

Instead, it is our strategy to study the implications of measurements at the LHC and the ILC by comparing to models over the full 24-dimensional parameter space of the MSSM described at the beginning of this section. The MSSM is also a restricted subspace of the space of all, completely general, supersymmetry models. However, as we will see, the large number of parameters of the MSSM allows a very wide range of scenarios to appear, with sufficient independence of parameters that measurements specific to the particles involved are needed to constraint the important physical effects. We believe that the exploration of this space is a reasonable way to estimate the capabilities of colliders to extract model-independent conclusions within the overall category of supersymmetry models. Many of the dependences of the neutralino relic density on individual MSSM parameters are displayed in [10], even though the conclusions are stated within the mSUGRA framework. Theoretical errors in the relic density calculation, which we do not consider in this paper, are also highlighted there. These errors become relevant when the relic density is determined to the $1 \%$ level. The dependence of the relic density on individual MSSM parameters at several of the reference points is also studied in [75]. Nojiri, Polesello and Tovey have recently redone the study [73] in the context of the full MSSM [11]. These results can be compared directly to ours, and we will do that in Section 4.3.

We conclude this section with a more technical explanation of our use of the MSSM parameters. First, there is ambiguity in how one would assign values to the 24 MSSM parameters from the output of ISAJET. We do this as follows: We first run ISAJET with the parameters in Table 1, and we run DarkSUSY at each point using roption='isasu' to use the running Yukawa couplings from ISAJET. From this, we extract the various running parameters evaluated at the mass scale $Q=\left(m\left(\widetilde{t}_{1}\right) m\left(\widetilde{t}_{2}\right)\right)^{1 / 2}$, which ISAJET uses as the mass scale of supersymmetry. These include the top, bottom and tau masses (MTQ, MBQ, MLQ), the Higgs vacuum expectation values $\left(\mathrm{VUQ}^{2}+\mathrm{VDQ}^{2}\right)^{1 / 2}$, the strong coupling constant GSS(3), and the Yukawa couplings GSS $(4,5,6)$. In addition, the 24 MSSM parameters listed above are extracted at the SUSY scale, with the exception of $m_{A}$ which we take as the physical mass, $\tan \beta$ which we take as the input value, and $m_{3}$ which we take as the physical gluino mass. We now run the benchmark model again using a low energy treatment in DarkSUSY. The spectrum is calculated according to the ISAJET function ssmass, from which we extract the neutralino, chargino, sfermion, and Higgs masses and mixings. Then DarkSUSY computes the relic density and other quantities. This is the benchmark point that we use. This procedure defines the benchmark point, yielding the reference spectra and the values of $\Omega_{\chi} h^{2}$ listed in Table 1. To describe a 
point in the more general parameter space of the MSSM, we compute the spectrum from ISAJET with ssmass using the new values of the 24 MSSM parameters, taking MTQ, MBQ, MLQ, the Higgs VEV, and the strong coupling constant GSS(3) to be fixed at the benchmark value. We take the Yukawa couplings GSS $(4,5,6)$ scaled by $\tan \beta$ from the benchmark values. For example, for LCC3 we take GSS(4) to be the benchmark value times $\left(\left(1+\tan ^{2} \beta\right) /\left(1+(40)^{2}\right)\right)^{1 / 2}$. With these choices, we describe the MSSM with low energy parameters in such a way that a point in our parameter space gives a close match to the input mSUGRA model.

\subsection{Scanning of parameter space}

We have now reduced the prediction of the properties of WIMPs to the following problem: Given a parameter space of $n$ coordinates $x$, and given a set of measurements $m_{i}$, each with standard deviation $\sigma_{i}$, what is the expectation for the prediction for additional observable quantities $O_{j}$ that depend on the parameters $x$ ? In Bayesian statistics, the probability distribution of $x$ is given by the likelihood function

$$
d^{n} x \mathcal{L}(x)=d^{n} x \prod_{i} \exp \left[-\frac{\left(M_{i}(x)-m_{i}\right)^{2}}{2 \sigma_{i}^{2}}\right] .
$$

We have made the assumption of a flat a priori distribution of the values of $x$, and we have assumed that the distribution of measurement errors is Gaussian. The prediction for $O_{j}(x)$ is then given by the expectation value of this function of $x$ in the measure (9). More generally, we can consider the distribution of the values of $O_{j}(x)$ induced by this distribution of the parameters $x$. In the next few sections, we will use this method to present the distributions of the WIMP relic densities and cross sections that follow from the constraints imposed by supersymmetry spectrum measurements.

A simple way to generate the distributions (9) is to choose points $x$ randomly in the parameter space and assign each one the indicated weight. However, if some of our measurements are very precise, the Gaussian distributions in (9) will be very steep and important points will be selected only rarely. A much more effective method for sampling points is the Markov Chain Monte Carlo (MCMC) method [76-80]. From an initial starting configuration $x_{0}$, we generate a sequence of points $x_{I}$ by the following algorithm: Choose a nearby point $x^{\prime}$. If $\mathcal{L}\left(x^{\prime}\right) \geq \mathcal{L}\left(x_{I}\right)$, let $x_{I+1}=x^{\prime}$. If not, let $x_{I+1}=x^{\prime}$ with probability $\mathcal{L}\left(x^{\prime}\right) / \mathcal{L}\left(x_{I}\right)$; otherwise, let $x_{I+1}=x_{I}$. This process satisfies detailed balance and therefore should equilibrate to an ensemble of points in which each point appears with the relative weight $\mathcal{L}(x)$.

There are many possible ways to choose the distribution of initial conditions, step sizes, and criteria for convergence in the MCMC algorithm. In our use of this

algorithm, we have chosen a reference point $X_{*}$ to be one of the LCC points, we 
have set the central values of measurements $m_{i}$ in the likelihood function to be the values $M_{i}\left(X_{*}\right)$ predicted at this point, and we have taken the initial point of the chain to be the reference point: $x_{0}=X_{*}$. In choosing step sizes, we have attempted to measure the size of the distribution of points $\left\{x_{I}\right\}$ and to readjust the step size so that it is comparable to this size in each dimension. For each reference point, we have generated 25 independent chains, with 160,000 points $x_{I}$ per chain, for a total of 4 million points.

We have tested our method by performing MCMC scans in which the step size has been preset from physical arguments and by performing flat scans of the parameter space in the vicinity of the reference points. The distributions near the reference points are similar in the three methods. The final errors estimated by the MCMC method are larger, but we are convinced that this is due to the fact that the MCMC scans explore the parameter space more deeply.

The full details of our MCMC algorithm are presented in Appendix A. It is easy to obtain apparently excellent but incorrect results with MCMC when the chains do not come to equilibrium. In Appendix A, we describe direct tests and cross-checks that have convinced us that our MCMC chains have correctly converged to the true likelihood distribution.

\subsection{Parameters and constraints}

For each of the four LCC points, we have scanned the 24-parameter space of the MSSM and obtained points distributed according to the appropriate likelihood function (9). In performing these scans, we have applied some general constraints that we will now describe.

For most of the 24 parameters, we have taken the prior distribution to be flat on a logarithmic scale: $d x p(x)=d x / x$. For parameters that can take either sign, we use a prior distribution flat in $\sinh ^{-1}(x / \mathcal{M})$, that is, $d x p(x)=d x / \sqrt{\mathcal{M}^{2}+x^{2}}$, with $\mathcal{M}=50 \mathrm{GeV}$. The latter are $A_{\tau}, A_{b}, A_{t}, \mu$ and $m_{1}$. The signs of the neutralino mass parameters $\mu$ and $m_{1}$ are discussed below. Given a set of MSSM parameters, we have used ISAJET 7.69 to compute the supersymmetry spectrum and mixing angles, in the manner that we have described at the end of Section 3.2. The ISAJET computation includes finite one-loop radiative corrections, assuming the MSSM parameters to be renormalized parameters at the scale $Q$ defined in that discussion. When we have required cross sections for supersymmetric particle production, we have computed these from the ISAJET output parameters using the tree level formulae.

For slepton masses and color-singlet gaugino masses, we have imposed a lower bound $m>100 \mathrm{GeV}$; for squark and gluino masses, we have imposed $m>250 \mathrm{GeV}$, except that $m(\widetilde{t})>150 \mathrm{GeV}$. These lower limits were almost always irrelevant, since 
measurements at the LHC and ILC would provide stronger lower bounds. However, we also needed to provide upper bounds to the parameter space; to do this, we restricted all mass parameters to be less than $5 \mathrm{TeV}$ in absolute value. We also imposed the following restrictions: For the parameter $\tan \beta$ : $2<\tan \beta<100$; for the chargino mass: $m\left(\chi_{1}^{+}\right)>125 \mathrm{GeV}$, assuming that an excess of trilepton events is not observed at the Tevatron. For the $A$ parameters, we have used

$$
A_{t}^{2}+\mu^{2}<7.5\left(m^{2}\left(\widetilde{t}_{1}\right)+m^{2}\left(\widetilde{t}_{2}\right)\right)
$$

as an approximate criterion to forbid charge- and color-breaking vacuum states [81]. We imposed no additional bounds on $A_{b}$ and $A_{\tau}$ since the top constraint is the most stringent. We did not implement the constraint from $b \rightarrow s \gamma$, since the predictions for $b \rightarrow s \gamma$ depend on flavor-mixing parameters that we are not including in our analysis. In our examples, however, the collider measurements of the supersymmetry spectrum exclude the region in which a large deviation in $b \rightarrow s \gamma$ is expected in models with minimal flavor violation.

The restriction to positive $m_{1}, m_{2}$, and $\mu$ deserves further comment. It is possible to choose $m_{2}$ to be positive by convention, but then $m_{1}$ and $\mu$ can in principle have either sign. It is usually very difficult to determine these signs from the LHC data. This is a special case of a more general problem, that spectrum constraints from the LHC often allow a number of interpretations in terms of the underlying supersymmetry parameters, related by discrete interchange operations. These 'discrete ambiguities' have been highlighted in $[82,83]$. We will see many examples of such ambiguities in the simulation results that we will present below, and we have studied in detail that our simulations count the multiple solutions correctly. Our conclusion about the signs of $m_{1}$ and $\mu$ is that allowing negative signs makes little difference to the results that we will show for the LHC, and that the ILC can typically distinguish the solution with positive signs. We will discuss this issue in more detail is Appendix B.

At the LHC, cross sections depend mainly on particle masses and so are useful only as cross-checks on the mass determinations. However, the electroweak cross sections at the ILC are often sensitive to mixing angles and provide additional information about the spectrum parameters. We have therefore included in our sets of measurements a number of ILC cross sections. The simulation studies for LCC1, LCC2, and LCC3 include estimates of the error that would be obtained on the total cross sections for some of the interesting reactions at these points.

The ILC will have polarized electron beams and probably also polarized positron beams. The cross sections have strong polarization-dependence. With this in mind, we have considered separately the cross sections for two different initial beam configurations: left-handed electron beam polarization with right-handed positron beam 
polarization and right-handed electron beam polarization with left-handed positron beam polarization, assuming polarizations of $80 \%$ for the electron beam and $60 \%$ for the positron beam. (Almost identical results are obtained with $95 \%$ electron polarization and zero positron polarization.) For certain processes, especially, $e^{+} e^{-} \rightarrow \chi_{1}^{+} \chi_{1}^{-}$, there is additional information in the forward-backward asymmetry. In these cases, we have divided the statistics between forward production $(\cos \theta>0)$ and backward production $(\cos \theta<0)$ and assigned errors to the two cross sections accordingly.

We have computed cross sections ignoring beamstrahlung and initial state radiation, and, for the cases with forward-backward asymmetries, ignoring possible problems in determining $\cos \theta$ from the decay final states. Where the error $\Delta \sigma$ was not available from simulation studies, we took the estimated error on the total cross section for a given polarization setting to be

$$
\Delta \sigma / \sigma=1 /\left(0.02 \times 250 \mathrm{fb}^{-1} \times \sigma\right)^{1 / 2},
$$

that is, the purely statistical error assuming $2 \%$ acceptance and a data sample of 250

$\mathrm{fb}^{-1}$ per polarization state. This estimate is typically conservative with respect to the results of the simulation studies.

\subsection{Importance sampling}

It is often useful to apply new constraints on a sample set after the calculation is complete. This amounts to adding new terms to the likelihood function $\mathcal{L}$. While the points in the Markov chain are distributed properly according to the original likelihood function, it is possible to re-weight them so that they are distributed according to a new likelihood function $\mathcal{L}^{\prime}$. This procedure is called importance sampling. If the two likelihood functions are very different, the reweighted chains will have poor statistics, so care must be taken. The basic procedure is very simple. Rather than counting each point in the chain once in computing statistics, count it $\mathcal{L}^{\prime} / \mathcal{L}$ times. Points that are highly unlikely according to $\mathcal{L}^{\prime}$ are thus counted with a very small weight.

We use importance sampling to apply astrophysical constraints to our datasets. We have considered constraining the relic density to agree with the dark matter density as measured from the CMB, and constraining the direction cross section to that corresponding to a direct detection signal and the assumption of a canonical smooth halo model.

For the relic density, we take the reweighting factor is to be

$$
\frac{\mathcal{L}^{\prime}}{\mathcal{L}}=\exp \left(-\frac{\left(\Omega h^{2}-\Omega h_{\mathrm{CMB}}^{2}\right)^{2}}{2 \sigma^{2}}\right),
$$


with a maximum value of unity. In the examples of the following sections, we have used a $3 \%$ constraint on relic density, corresponding to the design sensitivity of WMAP. This illustrates the effect of relic density constraints without having too harsh an effect on the statistical significance of the results.

When we impose constraints from direct detection, we are trying to extract information about the supersymmetry particle masses. For this, we must convert the observed number of events $n_{\mathrm{ev}}$ into a cross section. To do that, we must divide by the effective luminosity assumed in the analysis. In the analyses of the SuperCDMS experiment described in Section 4.5, the effective integrated luminosities for the four benchmark models are $L_{\mathrm{DD}}=3.80 \mathrm{zb}^{-1}, 3.60 \mathrm{zb}^{-1}, 3.12 \mathrm{zb}^{-1}, 2.79 \mathrm{zb}^{-1}$, respectively. It is appropriate to take different values for the four LCC points because the detection efficiency depends on the mass of the WIMP.

We use this information as follows to compute the new likelihood function: The expected number of events is $\tau=\sigma L_{\mathrm{DD}}$. We assume that the detection is a Poisson process and thus

$$
\mathcal{L}^{\prime}\left(n_{\mathrm{ev}} \mid \tau\right) \propto e^{-\tau} \tau^{n_{\mathrm{ev}}}
$$

We use Bayes' theorem to derive

$$
\mathcal{L}^{\prime}\left(\tau \mid n_{\mathrm{ev}}\right) \propto e^{-\tau} \tau^{n_{\mathrm{ev}}} P(\tau),
$$

where here $P(\tau)$ is the prior on $\tau$, which we assume to be constant. The reweighting factor can thus be taken as

$$
\frac{\mathcal{L}^{\prime}}{\mathcal{L}}=e^{n_{\mathrm{ev}}-\tau}\left(\frac{\tau}{n_{\mathrm{ev}}}\right)^{n_{\mathrm{ev}}}
$$

which again has a maximum value of unity.

\section{Benchmark point LCC1}

In this and the next few sections, we describe the predictions for the properties of the neutralino WIMP that would follow from collider measurements for the four LCC reference points. We will present the qualitative features of the analysis at each point and display relevant projections of the MCMC data. Our final quantitative results for the predictions of $\Omega_{\chi} h^{2}$ and other WIMP properties are collected in Tables 11 and 12 in Section 9.

We begin with the point LCC1. The supersymmetry spectrum at this point is shown in Fig. 7. The model contains light sleptons, with masses just above the mass of the lightest neutralino. The most important annihilation reactions for determining 


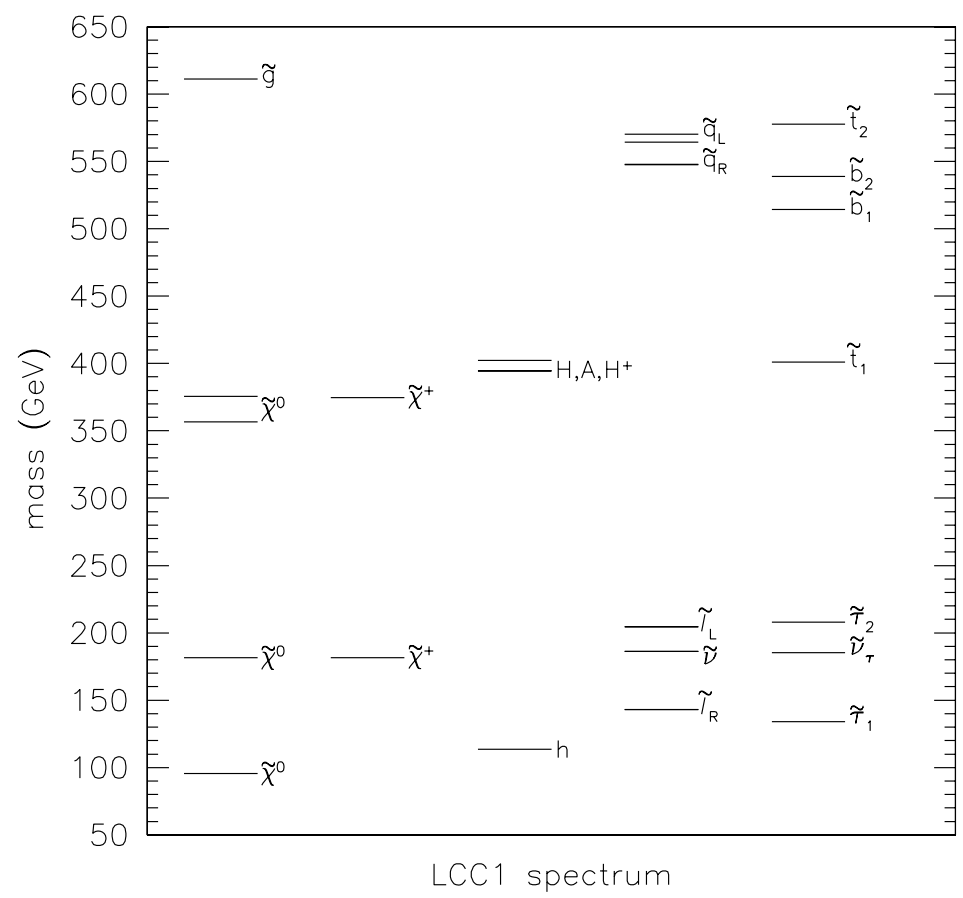

Figure 7: Particle spectrum for point LCC1. The lightest neutralino is predominantly bino, the second lightest neutralino and light chargino are predominantly wino, while the heaviest two neutralinos and heavy chargino are predominantly Higgsino.

the relic density are those with $t$-channel slepton exchange. To predict the relic density, we will need to make accurate measurements of the masses of the sleptons, including the $\widetilde{\tau}$.

Many other effects must be controlled to obtain the cross sections relevant to WIMP detection. The direct detection cross section is dominated by $H^{0}$ boson exchange, and the annihilation cross section at zero energy, which enters the rates for gamma ray and positron observations, is dominated by annihilation to $b \bar{b}$.

At the same time that we are gathering data to evaluate the dominant contributions to the WIMP cross sections, we must also gather data to prove that possible competing mechanisms from Fig. 6 are truly subdominant. In particular, we should use the data to show that neutralino annihilation does not take place close to an $A^{0}$ resonance, and that the wino and Higgsino content of the lightest neutralino is small enough that annihilation to vector bosons is not an important process. We will see that all of these factors can be controlled from the prospective collider data for the 
point LCC1.

\subsection{Spectroscopy measurements}

The basic data for determining the properties of the neutralino WIMP come from collider measurements of the SUSY spectroscopy. The measurements we assume are summarized in Tables 2 and 3. In general, we fix the central values for each observable to be equal to the prediction from the underlying model and fix the error (1 standard deviation) in accordance with the results of the simulation studies reported in [63].

The point LCC1 is special in that it allows a large number of SUSY spectrum parameters to be determined at the LHC from kinematic constraints. From Fig. 7, we can see that the lighter sleptons have masses between the masses of the $\widetilde{\chi}_{2}^{0}$ and the $\widetilde{\chi}_{1}^{0}$. In this case, the decays of the $\widetilde{\chi}_{2}^{0}$ will be dominated by 2 -body decays to $\tilde{\ell} \ell$, followed by the 2 -body decays $\tilde{\ell} \rightarrow \widetilde{\chi}_{1}^{0} \ell$. The kinematic endpoints of the lepton spectra in these decays are simple functions of the SUSY particle masses. The system is overconstrained, so it is possible to solve for the masses of the slepton and the two lightest neutralinos [84]. The values of the heavier superparticle masses can be built up from this information. Through this technique, the mass of the WIMP can be determined to about $5 \%$ accuracy, and the mass differences of the lighter neutralinos and sleptons can be measured to a few $\mathrm{GeV}$.

The squarks that are partners of left-handed quarks have large decay branching fractions to $\widetilde{\chi}_{2}^{0}$, so these mass differences can also be determined quite well. The partners of right-handed quarks can be studied using the direct decay to $\tilde{\chi}_{1}^{0}$. A similar analysis allows one to identify the right- and left-handed selectron and smuon states [63]. For stau, one mass eigenstate is seen, and this is a mixture of $\widetilde{\tau}_{L}$ and $\widetilde{\tau}_{R}$. The branching ratio of $\widetilde{\chi}_{2}^{0}$ to $\widetilde{\tau}$ is enhanced with respect to that to $\widetilde{e}, \widetilde{\mu}$ by the amplitude for the wino component of $\widetilde{\chi}_{2}^{0}$ to decay to the $\widetilde{\tau}_{L}$ component of $\widetilde{\tau}_{1}$. Thus, the measurement of this branching ratio gives information on the stau mixing angle [11].

In the study [63], it was not possible to identify the top squark at the LHC. However, this particle would have been visible through its decay $\widetilde{t}_{1} \rightarrow W^{+} b \widetilde{\chi}_{1}^{0}$ if the decay to $t \widetilde{\chi}_{1}^{0}$ were kinematically forbidden. We therefore place a limit $m\left(\widetilde{t}_{1}\right)>$ $m_{t}+m\left(\tilde{\chi}_{1}^{0}\right)$.

The light Higgs boson in the model has a mass of $114 \mathrm{GeV}$ and properties very similar to those of the Standard Model Higgs boson. It can be observed at the LHC in its decay to $\gamma \gamma$. This particle plays a relatively small role in the properties of the WIMP. However, the heavy Higgs boson $A^{0}$ can have a more important effect, since it potentially gives a resonant enhancement of the annihilation cross section. It is very significant, then, that the LHC can constrain the properties of the $A^{0}$ boson. If the $A^{0}$ is sufficiently light or if $\tan \beta$ is sufficiently large, the $A^{0}$ can be discovered at the 


\begin{tabular}{|c|c|c|c|c|c|}
\hline mass/mass splitting & LCC1 Value & & $\mathrm{LHC}$ & ILC 500 & ILC 1000 \\
\hline$m\left(\widetilde{\chi}_{1}^{0}\right)$ & 95.5 & \pm & 4.8 & 0.05 & \\
\hline$m\left(\widetilde{\chi}_{2}^{0}\right)-m\left(\widetilde{\chi}_{1}^{0}\right)$ & 86.1 & \pm & 1.2 & 0.07 & \\
\hline$m\left(\widetilde{\chi}_{3}^{0}\right)-m\left(\widetilde{\chi}_{1}^{0}\right)$ & 261.2 & \pm & $@^{a}$ & 4.0 & \\
\hline$m\left(\tilde{\chi}_{4}^{0}\right)-m\left(\tilde{\chi}_{1}^{0}\right)$ & 280.1 & \pm & $2.2^{a}$ & 2.2 & \\
\hline$m\left(\tilde{\chi}_{1}^{+}\right)$ & 181.7 & \pm & - & 0.55 & \\
\hline$m\left(\tilde{\chi}_{2}^{+}\right)$ & 374.7 & \pm & - & - & 3.0 \\
\hline$m\left(\widetilde{e}_{R}\right)$ & 143.1 & \pm & - & 0.05 & \\
\hline$m\left(\widetilde{e}_{R}\right)-m\left(\widetilde{\chi}_{1}^{0}\right)$ & 47.6 & \pm & 1.0 & 0.2 & \\
\hline$m\left(\widetilde{\mu}_{R}\right)-m\left(\widetilde{\chi}_{1}^{0}\right)$ & 47.5 & \pm & 1.0 & 0.2 & \\
\hline$m\left(\widetilde{\tau}_{1}\right)-m\left(\widetilde{\chi}_{1}^{0}\right)$ & 38.6 & \pm & 5.0 & 0.3 & \\
\hline$B R\left(\widetilde{\chi}_{2}^{0} \rightarrow \widetilde{e} e\right) / B R\left(\tilde{\chi}_{2}^{0} \rightarrow \widetilde{\tau} \tau\right)$ & 0.077 & \pm & 0.008 & & \\
\hline$m\left(\widetilde{e}_{L}\right)-m\left(\tilde{\chi}_{1}^{0}\right)$ & 109.1 & \pm & 1.2 & 0.2 & \\
\hline$m\left(\widetilde{\mu}_{L}\right)-m\left(\tilde{\chi}_{1}^{0}\right)$ & 109.1 & \pm & 1.2 & 1.0 & \\
\hline$m\left(\widetilde{\tau}_{2}\right)-m\left(\widetilde{\chi}_{1}^{0}\right)$ & 112.3 & \pm & - & 1.1 & \\
\hline$m\left(\widetilde{\nu}_{e}\right)$ & 186.2 & \pm & - & 1.2 & \\
\hline$m(h)$ & 113.68 & \pm & 0.25 & 0.05 & \\
\hline$m(A)$ & 394.4 & \pm & $*$ & $(>240)$ & 1.5 \\
\hline$m\left(\widetilde{u}_{R}\right), m\left(\widetilde{d}_{R}\right)$ & 548. & \pm & 19.0 & 16.0 & \\
\hline$m\left(\widetilde{s}_{R}\right), m\left(\widetilde{c}_{R}\right)$ & 548. & \pm & 19.0 & 16.0 & \\
\hline$m\left(\widetilde{u}_{L}\right), m\left(\widetilde{d}_{L}\right)$ & $564 ., 570$. & \pm & 17.4 & 9.8 & \\
\hline$m\left(\widetilde{s}_{L}\right), m\left(\widetilde{c}_{L}\right)$ & $570 ., 564$ & \pm & 17.4 & 9.8 & \\
\hline$m\left(\widetilde{b}_{1}\right)$ & 514 & \pm & 7.5 & 5.7 & \\
\hline$m\left(\widetilde{b}_{2}\right)$ & 539. & \pm & 7.9 & 6.2 & \\
\hline$m\left(\widetilde{t}_{1}\right)$ & 401. & \pm & $(>270)$ & - & 2.0 \\
\hline$m(\widetilde{g})$ & 611. & \pm & 8.0 & 6.5 & \\
\hline
\end{tabular}

Table 2: Superparticle masses and their estimated errors or lower limits for the parameter point LCC1. Lower limits are indicated in parentheses. The ILC columns contain the measurements added or improved by the ILC at that energy. The symbol '-' denotes that the measurement is not yet available. The symbol ' $*$ ' denotes the formula: $m_{A}>200 \mathrm{GeV}$, or $\tan \beta<7.0\left(m_{A} / 200.0\right)$. The notation '@ $a$ ' indicates that the mass measurement marked with a superscript ${ }^{a}$ could equally well be ascribed to this particle. All values are quoted in $\mathrm{GeV}$. 


\begin{tabular}{lccccc} 
cross section & & LCC1 Value (fb) & & ILC 500 & ILC 1000 \\
\hline$\sigma\left(e^{+} e^{-} \rightarrow \widetilde{\chi}_{1}^{+} \widetilde{\chi}_{1}^{-}\right)$ & LR & $431.5(0.758)$ & \pm & $1.1 \%^{*}$ & \\
& RL & $13.1(0.711)$ & \pm & $3.5 \%^{*}$ & \\
$\sigma\left(e^{+} e^{-} \rightarrow \widetilde{\chi}_{1}^{0} \widetilde{\chi}_{2}^{0}\right)$ & LR & 172.2 & \pm & $2.1 \%^{*}$ & \\
& RL & 20.6 & \pm & $7.5 \%^{*}$ & \\
$\sigma\left(e^{+} e^{-} \rightarrow \widetilde{\chi}_{2}^{0} \widetilde{\chi}_{2}^{0}\right)$ & LR & 189.9 & \pm & $2.0 \%^{*}$ & \\
& RL & 5.3 & \pm & $10.2 \%^{*}$ & \\
$\sigma\left(e^{+} e^{-} \rightarrow \widetilde{\tau}_{1}^{+} \widetilde{\tau}_{1}^{-}\right)$ & LR & 45.6 & \pm & $7 \%$ & \\
& RL & 142.1 & \pm & $4 \%$ & \\
$\sigma\left(e^{+} e^{-} \rightarrow \widetilde{e}_{R}^{+} \widetilde{e}_{R}^{-}\right)$ & $\mathrm{LR}$ & $57.3(0.696)$ & \pm & $6 \%$ & \\
& $\mathrm{RL}$ & $879.9(0.960)$ & \pm & $1.5 \%$ & \\
$\sigma\left(e^{+} e^{-} \rightarrow \widetilde{t}_{1} \widetilde{\bar{t}}_{1}\right)$ & $\mathrm{LR}$ & 9.8 & \pm & & $15 \%$ \\
& $\mathrm{RL}$ & 11.1 & \pm & & $14 \%$ \\
\hline
\end{tabular}

Table 3: SUSY cross sections and estimated errors for the parameter point LCC1. The ILC columns indicate the center of mass energy of the measurement. All cross sections assume polarized beams. LR denotes left-handed $e^{-}$polarization and right-handed $e^{+}$polarization; $\mathrm{RL}$ denotes the reverse. Cross section values are quoted in $\mathrm{fb}$; the error are expressed as a percentage of the value. For chargino and selectron production cross sections, the forward fraction is listed in parentheses. The errors labeled by * are taken from the study [63]; the others are estimated using (11).

LHC in its decays to $\tau^{+} \tau^{-}$or (in a slightly smaller region) to $\mu^{+} \mu^{-}$. At LCC1, the $A^{0}$ boson cannot be discovered at the LHC, but the failure to observe this particle constrains the region in which the $A^{0}$ boson plays a role in the WIMP properties.

At the ILC, we expect very precise mass measurements for all particles that can be pair-produced at the machine's center of mass energy. Measurements of particle masses can come from kinematic fitting of pair-production reactions and from dedicated threshold energy scans [63]. In Table 2, mass measurements from kinematic fitting are given as mass differences from the lightest neutralino, and mass measurements from threshold determinations are indicated as absolute mass measurements. Because the masses of the light charginos and neutralinos enter the expressions for endpoints of kinematic distributions at the LHC, the determination of squark and gluino masses are improved by the ILC data even though these particles are not observed directly at the ILC. This effect is reflected in Table 2.

The extension of the ILC to center of mass energies of $1000 \mathrm{GeV}$ allows us to observe several particles that were not accessible at the first stage of $500 \mathrm{GeV}$. These include the top squark and the second chargino. A particularly important addition is that of the $H^{0}$ and $A^{0}$ bosons, since the process $e^{+} e^{-} \rightarrow H^{0} A^{0}$ becomes kinematically accessible above $900 \mathrm{GeV}$. We estimate the accuracy of the $H^{0}$ and $A^{0}$ mass 
determinations from the results of the study [67]. This sharpened knowledge of the masses of $A^{0}$ and $H^{0}$ will have an important effect on the determination of several of the WIMP properties.

\subsection{Relic density}

Now we can put the constraints shown in Tables 2 and 3 into the machinery described in Section 3.2 to provide predictions of WIMP dark matter properties. We begin with the WIMP relic density, as computed from the spectrum using DarkSUSY 4.1. To see what the various data sets predict for this quantity, we generate the three likelihood distributions corresponding to the three sets of constraints in the Tables, including the cross section for the two ILC points. In Fig. 8, we show the projections of these three likelihood distributions onto the coordinate representing the relic density $\Omega_{\chi} h^{2}$. The LHC data already gives a quantitative prediction for this density, with an accuracy of about $7 \%$. The data from the $500 \mathrm{GeV}$ ILC sharpens this distribution to $2 \%$ accuracy, though the distribution has a long tail toward lower values. The extension of the ILC to $1000 \mathrm{GeV}$ further sharpens the prediction to about $0.25 \%$ accuracy, comparable to the accuracy expected from the Planck CMB measurement. Comparison of two such accurate values of the dark matter relic density would provide a striking test of the WIMP model, as we have noted already in Section 2.5.

One might have suspected that the large number of constraints from the LHC would already produce a very precise value of the relic density. However, it is clear from the spread of the LHC predictions in Fig. 8 that some information is missing. The scatter plots in Fig. 9(a,b) show one of the effects that contributes. Though the mass differences of the supersymmetry particles are well fixed, the overall scale of masses is somewhat uncertain. This translates into the dominant uncertainty in the prediction of the relic density.

The tail in the $\Omega_{\chi} h^{2}$ distribution from the $500 \mathrm{GeV}$ ILC is also a surprise. Its explanation is given in Fig. 9(c), which shows the correlation between $\Omega_{\chi} h^{2}$ and the

mass of the $A^{0}$ boson in this likelihood function. The $s$-channel process involving the $A^{0}$ boson actually makes a small contribution to the annihilation cross section at LCC1, and this must be fixed to determine the cross section to a level below $1 \%$. When we learn the mass of the $A^{0}$ at the $1000 \mathrm{GeV}$ ILC, this uncertainty in the prediction of $\Omega_{\chi} h^{2}$ is removed.

\subsection{Relic density at SPS1a'}

Nojiri, Polesello, and Tovey (NPT) [11] have also estimated the capability of the LHC data to predict the neutralino relic density. Their analysis was done at a 


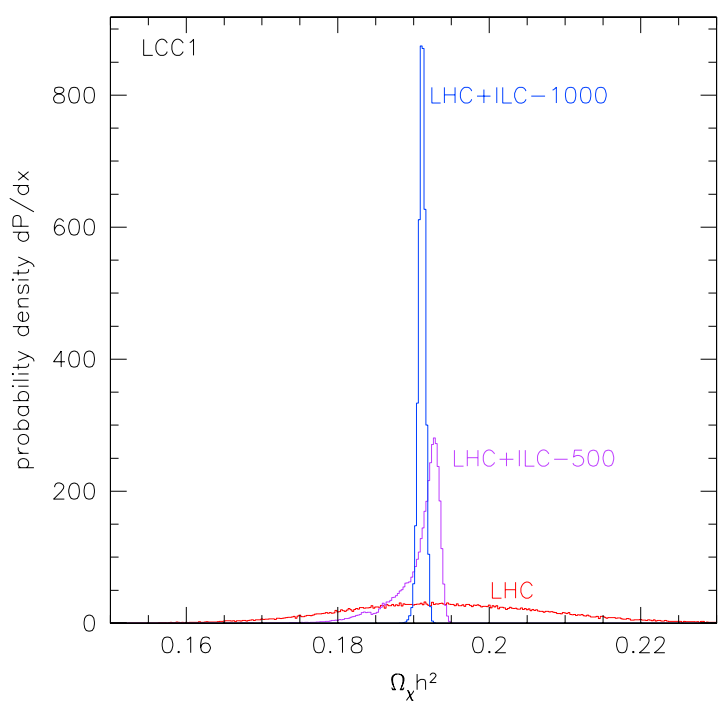

Figure 8: Relic density measurement for point LCC1. Histograms in this and all following figures give the probability distribution $d P / d x$ of the quantity on the $x$-axis, given the three different sets of accelerator constraints. Where the $x$ axis is plotted logarithmically, the probability plotted is actually $d P / d \log _{10} x$. All histograms integrate to unity. Results for the LHC make use of the assumption that the underlying physics model is supersymmetry. This might not be clear from the LHC data alone.

point quite similar, but not identical to, LCC1. By adjusting the parameters of our simulation, we can make a direct comparison of our results to those of NPT. In this section, we will describe our analysis of the point considered in [11] and show that our results are in good agreement. Since NPT used a different method to arrive at their estimate, this comparison provides a nontrivial check of the two methods.

The value of $\Omega_{\chi} h^{2}$ at LCC1 is about a factor of 2 too large to be consistent with the $\mathrm{CMB}$ result [1]. It has been suggested that one could remedy this problem by moving the mass of the $\widetilde{\tau}_{1}$ from $134 \mathrm{GeV}$ at LCC1 to $108 \mathrm{GeV}$, pushing the model into the stau coannihilation region. The new point is called SPS1a' [68]. The shift of the $\widetilde{\tau}_{1}$ mass adds contributions to the annihilation cross section for supersymmetry and therefore decreases the relic density. The neutralino physics at this point is intermediate between that at LCC1 and LCC3. Because coannihilation is important, the point has increased sensitivity to the value of the $\widetilde{\tau}_{1}$ mass, though this sensitivity is not as extreme as we will see in Section 6 in our discussion of LCC3.

According to [68], the table of measurement errors at SPS1a' should be the same as that of LCC1 (Tables 2 and 3), even though the expected values are somewhat 

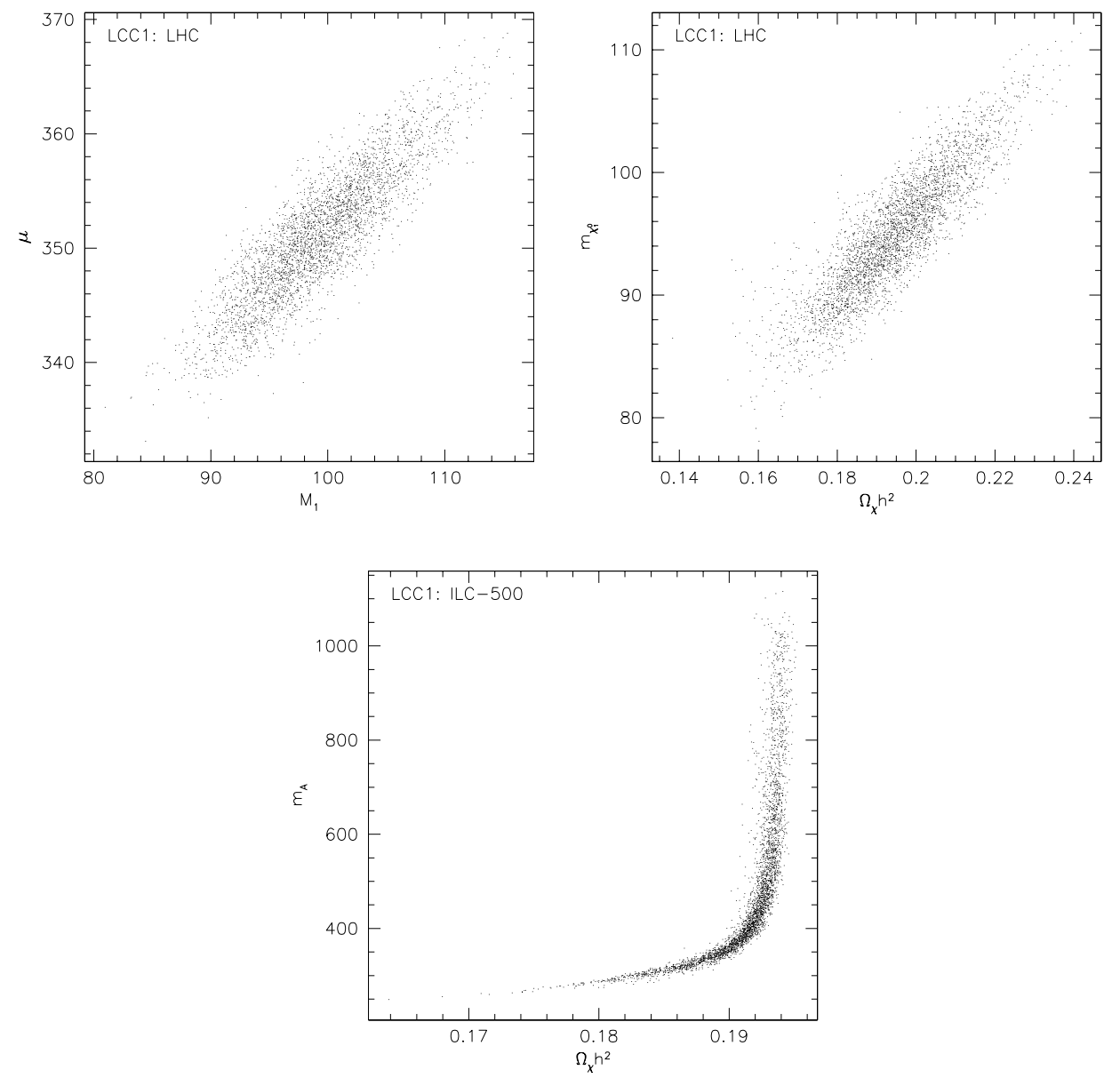

Figure 9: Scatter plots for point LCC1. (a) Inferred mass parameters $m_{1}$ and $\mu$ are shown to be well correlated in LHC data, with an uncertain overall scale. This reflects the fact that mass differences are measured more precisely than absolute masses. (b) The overall scale has a strong correlation with relic density. (c) For ILC-500, the mass of the $A^{0}$ boson is still unknown. For low values, there is a mild resonant enhancement to the neutralino annihilation cross section, and a corresponding reduction in relic density. 
different. We have therefore carried out MCMC scans for SPS1a' using the same constraints as for SPS1a (LCC1), only modifying the reference values of supersymmetry masses and cross sections. The results of that scan for the relic density are shown in Fig. 10 .

The analysis of NPT ran as follows: In the stau coannihilation region, the neutralino relic density is mainly sensitive to 7 of the 24 MSSM parameters, the four parameters of the neutralino mass matrix $m_{1}, m_{2}, \mu$, and $\tan \beta$ and the three parameters of the stau mass matrix, which we can represent as the combinations $m\left(\widetilde{\tau}_{1}\right)$, $m\left(\widetilde{\tau}_{2}\right), \theta_{\tau}$. The LHC data is expected to give relatively precise values for five quantities sensitive to these parameters, three neutralino masses, the mass of the $\widetilde{\tau}_{1}$, and the ratio of branching ratios $B R\left(\widetilde{\chi}_{2}^{0} \rightarrow e \widetilde{e}\right) / B R\left(\widetilde{\chi}_{2}^{0} \rightarrow \tau \widetilde{\tau}_{1}\right)$. At the true point, the gaugino-Higgsino and stau mixing angles are relatively small, so the actual dependence of the relic density on the mixing angles (and thus on $\tan \beta$ ) is relatively weak. The dependence on $m\left(\widetilde{\tau}_{2}\right)$ is also weak. So it makes sense in this case to solve for the five parameters $m_{1}, m_{2}, \mu, m\left(\widetilde{\tau}_{1}\right)$, and $\theta_{\tau}$ from the measurements, propagate the measurement errors through to an error on the prediction of $\Omega_{\chi} h^{2}$, and then add an estimate of the additional uncertainty from variation of $\tan \beta$ and $m\left(\widetilde{\tau}_{2}\right)$. In solving for the five parameters, NPT chose the preferred solution and did not take account of the possibility of multiple solutions.

The final error on the relic density turned out to be dominated by the uncertainty in the mass of of the $\widetilde{\tau}_{1}$. This is tied to the error in measuring the endpoint of the dilepton mass spectrum in the decay $\widetilde{\chi}_{2}^{0} \rightarrow \tau^{+} \tau^{-} \widetilde{\chi}_{1}^{0}$. For the case of tau leptons, the endpoint is not a sharp feature and it has not been understood in detail how well this point can be measured. For the purpose of their analysis, Polesello and Tovey, who are two of the experts on such measurements in the ATLAS collaboration, estimated that the error would be below $5 \mathrm{GeV}$ and expressed the hope that it could be brought down to $1 \mathrm{GeV}$. This is considerably more aggressive than the uncertainty presented in [63], which we have assumed in our study.

In Fig. 11, we show the histograms of $\Omega_{\chi} h^{2}$ from the LHC constraints for our scan with parameters based on [63] and imposing the stronger constraints that the $\tau \tau$ endpoint can be measured to $5 \mathrm{GeV}$ or to $1 \mathrm{GeV}$. From the figure, it is clear that NPT are correct that this is the dominant source of error. The standard deviations for the three histograms correspond to

$$
\sigma\left(\Omega h^{2}\right) / \Omega h^{2}=18.6 \%, 14.4 \%, 11.7 \%
$$

with the last two cases to be compared to the results of NPT,

$$
\sigma\left(\Omega h^{2}\right) / \Omega h^{2}=19 . \%, 10.5 \%
$$

Their results correspond to a scan over 5 parameters, while our results scan over the full set of 24 MSSM parameters. We thus verify that the analysis of NPT does 


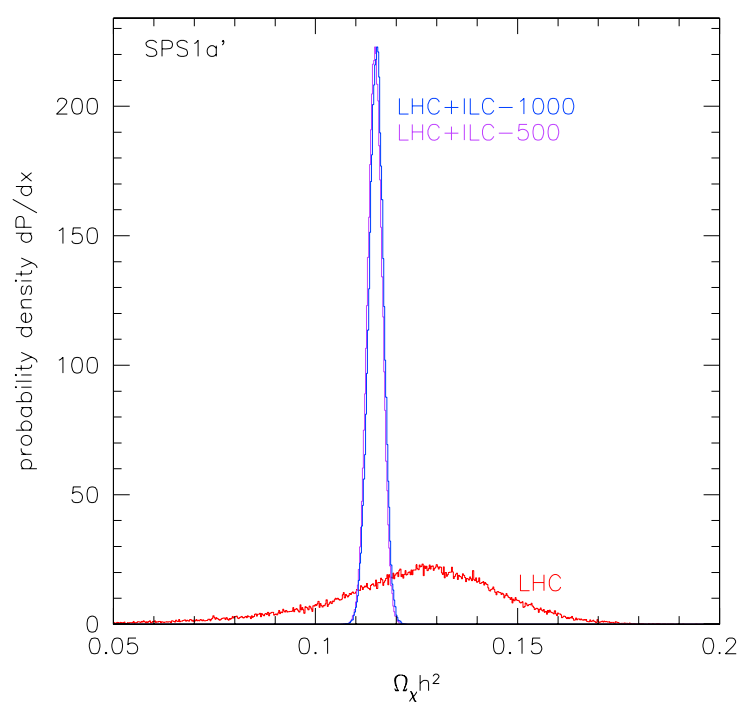

Figure 10: Relic density for point SPS1a'. See Fig. 8 for description of histograms. In this case, the ILC-1000 provides no improvement in the relic density measurement over ILC-500.

capture the strongest dependences of $\Omega h^{2}$ on the MSSM parameters, and also that our method for estimating the uncertainty in $\Omega_{\chi} h^{2}$ does agree with a more direct method in this case where $\Omega_{\chi} h^{2}$ is strongly constrained. In Fig. 12, we show the scatter plot of $\Omega_{\chi} h^{2}$ vs. $m\left(\widetilde{\chi}_{1}^{0}\right)$ from our scan for the case of a $1 \mathrm{GeV}$ measurement of the $\tau \tau$ endpoint. This is, again, a scan over 24 parameters. It is interesting to compare this to Fig. 9 of NPT, which gives the result of a 5-parameter scan.

Given the very strong constraints on the spectrum at SPS1a', the ambiguities in the solutions play a rather small role. in particular, the constraint on the ratio of branching ratios of the $\widetilde{\chi}_{2}^{0}$ almost completely eliminates the solution in which the lighter $\widetilde{\tau}$ is dominantly $\widetilde{\tau}_{L}$. Our data contains only a small influence of the region in which the $A$ boson provides a resonant enhancement of the neutralino annihilation. Fig. 13 shows a scatter plot $\Omega_{\chi} h^{2}$ vs $m(A)$ for the SPS1a' LHC data sample. We see a small branch leading to low $m(A)$ and low $\Omega_{\chi} h^{2}$, representing the effect of an $A$ boson resonance near the annihilation threshold.

\subsection{Annihilation cross section}

In Fig. 14, we show the likelihood distribution of the neutralino pair annihilation cross section times velocity $\sigma_{\chi \chi} v$, evaluated at threshold. As we have discussed in Section 2.7, this cross section is needed to interpret astrophysical signals of dark 


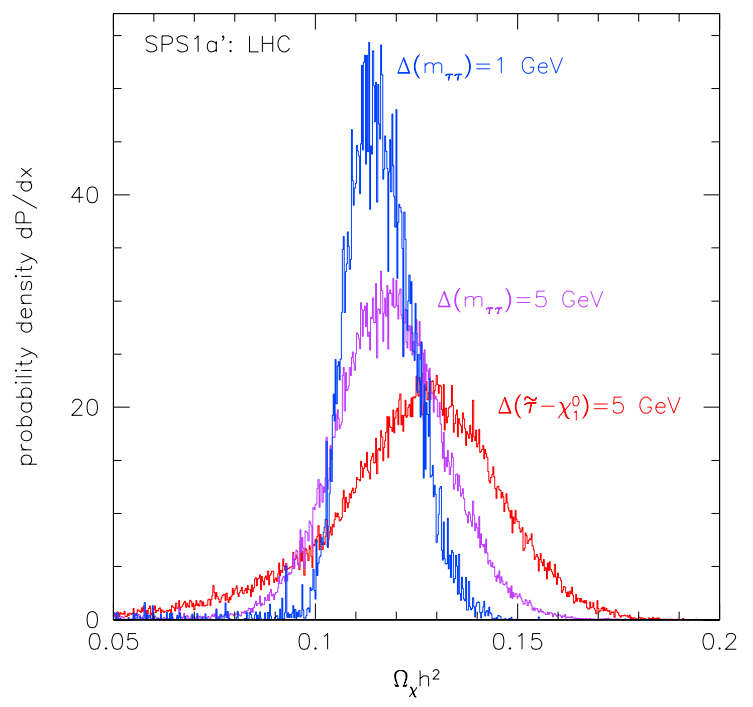

Figure 11: Relic density for point SPS1a ${ }^{\prime}$ at LHC. A dominant uncertainty at this point is the mass of the stau. Results of taking the stau-neutralino mass difference to have an error of $5 \mathrm{GeV}$ are illustrated in red. Alternatively, results of taking the position of the tau-tau edge to have an error of $5 \mathrm{GeV}$ (purple) and $1 \mathrm{GeV}$ (blue) are also illustrated.

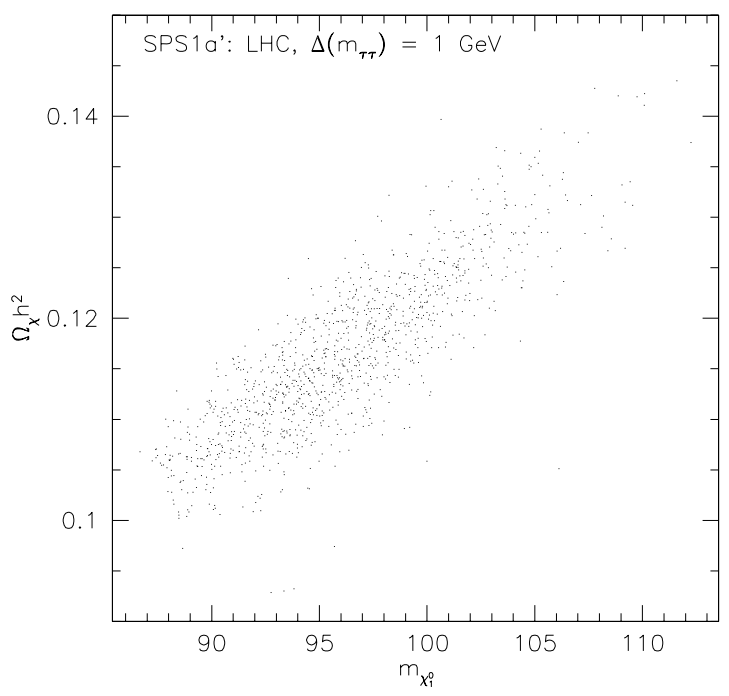

Figure 12: Scatter plot for point SPS1a'. Relic density is plotted against neutralino mass, for points where the position of the tau-tau edge is measured to $1 \mathrm{GeV}$. 


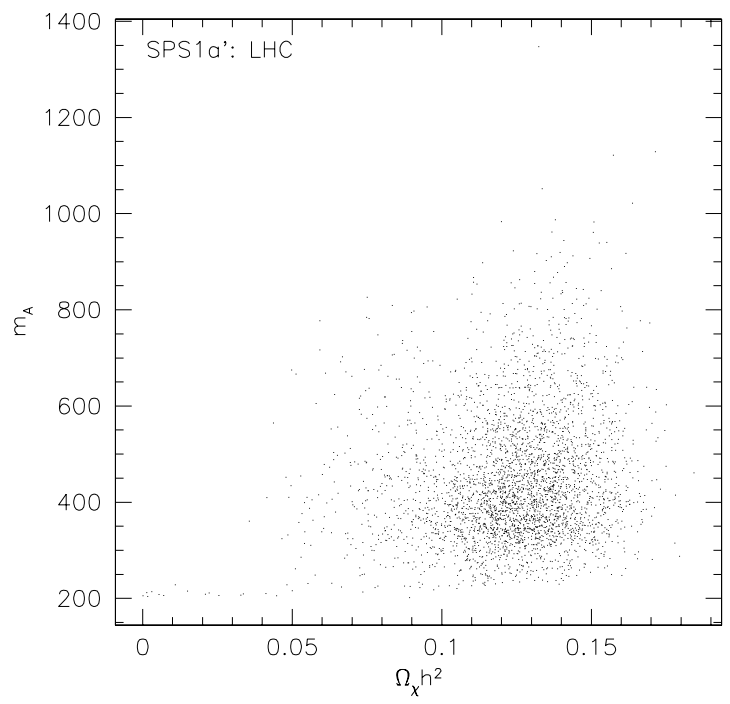

Figure 13: Scatter plot for point SPS1a'. The $A^{0}$ mass is plotted against relic density, illustrating the small allowed region where $A^{0}$ resonance significantly decreases the relic density.

matter annihilation. We will discuss the determination of this quantity from a more general point of view in Section 8 .

At LCC1, the most important neutralino annihilation reactions are P-wave processes whose cross section vanishes at threshold. The important contributions to the threshold cross sections then come from annihilation to massive fermion pairs, $\tau^{+} \tau^{-}$ and $b \bar{b}$, with the latter final state dominating. The most important parameters for $\widetilde{\chi}_{1}^{0} \widetilde{\chi}_{1}^{0} \rightarrow b \bar{b}$ are the $\widetilde{b}$ masses; at LCC1, these masses would be determined at the LHC. The ILC at $500 \mathrm{GeV}$ determines the neutralino mixing angles, and this makes a small improvement in the prediction. The marked improvement from the ILC at $1000 \mathrm{GeV}$ comes from the determination of the $A^{0}$ mass. Note that, because of the suppression of the $\mathrm{P}$-wave channels, this annihilation cross section is about $12 \mathrm{fb}$, as opposed to the $0.9 \mathrm{pb}$ predicted by $(2)$.

In Fig. 14, we show the likelihood distribution for the exclusive annihilation cross sections to $\gamma \gamma$ and $\gamma Z^{0}$. These cross sections are dominated by squark loops and are determined quite well already from LHC data. The cross sections are small, but, at LCC1, these processes might be visible as sharp lines in the gamma ray spectrum.

In Fig. 16, we present two representative calculations that indicate how this information might be used. We will postulate two situations in which dark matter might 
be detected by gamma rays from annihilation, and we will work through the numbers to see how accurately the absolute dark matter density can be obtained from these observations. Because of the intrinsically small annihilation cross sections at LCC1, the final results that we will show in this section will be rather marginal. In later sections, we will present the same calculations for the other three reference points. At points LCC2 and LCC4, the annihilation cross section at threshold is 50 times larger, leading to much more optimistic expectations for dark matter detection.

First, we consider the dark matter distribution at the galactic center, assuming the NFW distribution shown in Fig. 5. We set the boost factor $B=1$. Observation in a circle of angular area $10^{-1}$ sr gives $\langle J(\Omega)\rangle=100$. We use this value to predict the rate of gamma ray emission from this source. To do this, we use the spectrum of gammas expected for neutralino annihilation at LCC1, scaled to the predicted total annihilation cross section. (We will describe this spectrum in Section 8.1.) We impose a lower energy cutoff, $E_{\gamma}>1 \mathrm{GeV}$, and add the galactic background in the same energy region as parametrized by Bergstrom, Ullio, and Buckley [85]. A fiveyear observation by the GLAST gamma-ray observatory [86], in which the galactic center is visible $20 \%$ of the time, yields an expectation of 199 signal photons over a background of 43,000 background gamma ray photons. This is a $S / \sqrt{B}$ of about 1 , so in principle we have some sensitivity to the effect.

We can now work backwards to determine the source strength $\langle J(\Omega)\rangle$ from the observation. For the purpose of this exercise, we assign the background rate a $5 \%$ systematic uncertainty. In pratice, it will be a challenge to obtain such a good understanding of the background. Gamma rays come from the galactic center from many sources, and, in particular, from energetic processes associated with the black hole at the center of galaxy. However, gamma rays from $\pi^{0}$ 's produced in soft hadronic interactions have a soft power-law energy spectrum different from that predicted for neutralino annihilation. In addition, the observed gamma rays seem to be associated with the molecular clouds that ring the galactic center rather than the having a peak at the center itself [87]. This observation has already been used to improve the limits on gamma rays from the galactic center from the EGRET observatory [88]. Thus, we believe that the detailed surveys of the position- and energy-dependence of gamma ray sources near the galactic center that will be done over the next ten years can reach the level of understanding that can separate gamma ray sources from high-energy astrophysical processes from that of neutralino annihilation at this level [89].

For the case of LCC1, even this aggressive estimate makes the uncertainty in the background the dominant source of error. The likelihood distribution for $\langle J(\Omega)\rangle$ that we obtain from this analysis is shown in Fig. 16(a). In this case, we do not obtain a prediction of $\langle J(\Omega)\rangle$, but we do obtain a strong upper limit that excludes otherwise viable models predicting large values of $J(\Omega)$. 


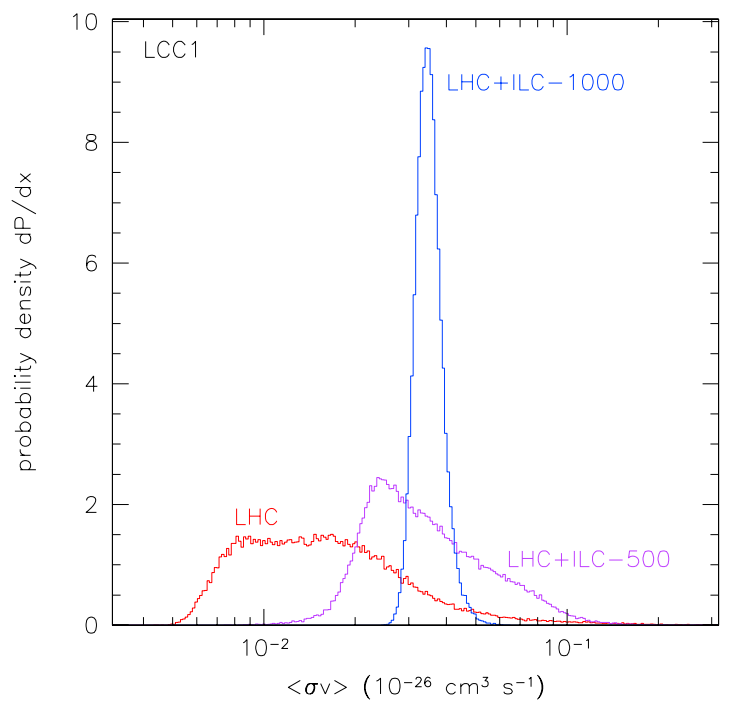

Figure 14: Annihilation cross section at threshold for point LCC1. See Fig. 8 for description of histograms.

In models in which the galaxy is build up from hierarchical dark matter clustering, one should expect to find localized clumps of dark matter. We choose a typical object from the semi-analytic simulation data of Taylor and Babul [53], a dark matter clump with a mass of $10^{6} M_{\odot}$ and a scale radius of $500 \mathrm{pc}$, at a distance of $6 \mathrm{kpc}$, with its internal structure described by an NFW profile. Observing in a disk of $3 \times 10^{-6}$ sr, the object has $\langle J(\Omega)\rangle=10^{4}$. Assuming a five-year observation by the GLAST observatory, in which this object would be visible $20 \%$ of the time, we expect 3 signal photons, plus 6 photons from the extra-galactic background at the level measured by EGRET [90]. Working backwards from the observations, assuming that the extragalactic background is well characterized from observations in other regions of the sky, we find the likelihood distribution of $\langle J(\Omega)\rangle$ shown in Fig. 16(b). Given the ratio of signal/background, we can only impose an upper limit on $\langle J\rangle$, but this still has the power to exclude models with very strongly peaked dark matter clumps.

Certainly, the discovery of a localized clump of dark matter in gamma rays would be remarkable in itself. But it would be more remarkable if we could obtain from particle physics a calibration of the absolute scale of its dark matter density. Fig. 16(b) shows that this is possible in principle. At the other benchmark points, which give larger gamma ray fluxes from neutralino annihilation, we will see this idea realized more clearly. 


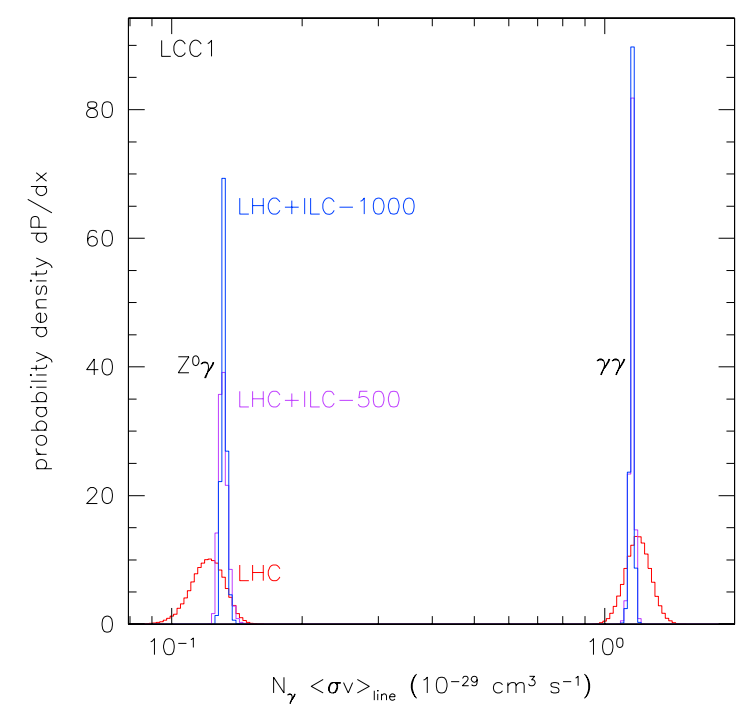

Figure 15: Gamma ray line annihilation cross section at threshold for point LCC1. See Fig. 8 for description of histograms.

\subsection{Direct detection cross section}

In Fig. 17, we show the likelihood distributions of the spin-averaged neutralinoproton cross section $\sigma_{\chi p}$, evaluated at threshold. This cross section is sufficiently close to the spin-independent isoscalar cross section that it can be used to interpret signals of direct detection of dark matter in underground detectors. The corresponding distributions for the spin-dependent part of the cross section $\sigma_{\chi n}$ are shown in Fig. 18.

From Fig. 17, it is clear that neither the LHC nor the ILC at $500 \mathrm{GeV}$ can make a particularly accurate prediction of this cross section. At LCC1, the direct detection cross section is dominated by the $t$-channel exchange of the heavy Higgs boson $H^{0}$. In the MSSM, the mass of the $H^{0}$ is very close in mass to the $A^{0}$ unless both are light. Even with the ILC data at $500 \mathrm{GeV}$, this mass is essentially unconstrained. The sharp edge in the distributions at $10^{-9} \mathrm{pb}$ reflects the contribution of the light Higgs $h^{0}$. Fig. 19, which displays the correlation in the LHC likelihood function between the neutralino-proton cross section and the heavy Higgs boson mass, shows clearly that this parameter is the essential missing piece of information It also illustrates the fact that above about $1.5 \mathrm{TeV}$, the cross section is insensitive to the heavy Higgs mass as the light Higgs contribution is of a comparable size. The slight improvement from the LHC to the $500 \mathrm{GeV}$ ILC reflects the determination of neutralino mixing angles, which enter the neutralino-Higgs vertices. Indeed, when the $H^{0}$ and $A^{0}$ particles are 

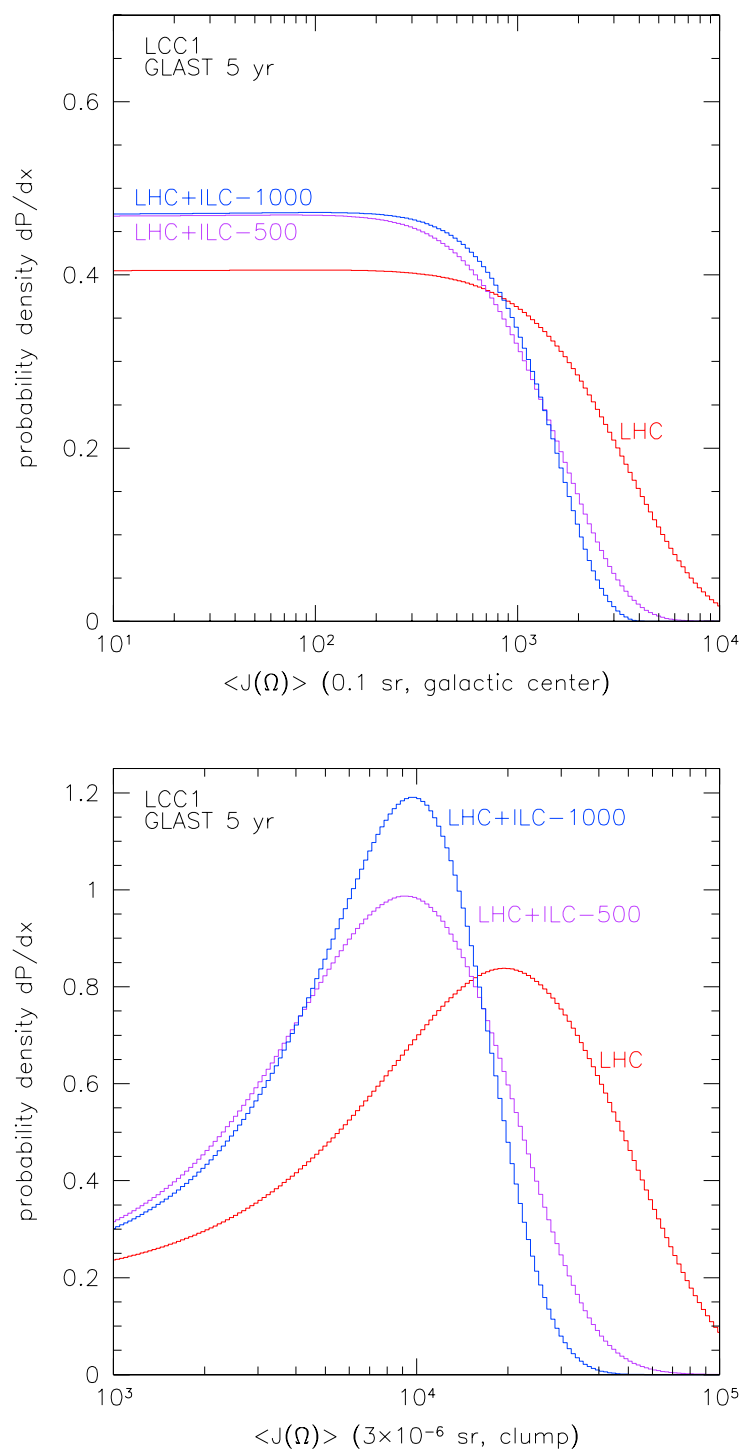

Figure 16: Halo density profiles for point LCC1: (a) galactic center, (b) dark matter clump in the galactic halo. Angle-averaged $J$ values as measured by combining a 5 -year all-sky dataset from GLAST with accelerator measurements are shown. See Fig. 8 for description of histograms. 
observed and measured at the $1000 \mathrm{GeV}$ ILC, we obtain a prediction of the direct detection cross section to about $20 \%$ accuracy.

As in the previous section, it is illuminating to work through an example of this application of this cross section determination to experimental data. At the point LCC1, dark matter events would not be seen in the CDMS II experiment, but the signal should be discovered in the next-generation detector SuperCDMS or "SuperCDMS 25kg' [42]. This is a $26.67 \mathrm{~kg}$ detector on the model of CDMS, with most of the mass in Germanium, to be located in the low-background environment of the Sudbury mine. Estimates of the detection capabilities of SuperCDMS were computed assuming 2.5 years of operation (ending in 2011) with a total exposure of close to $16,000 \mathrm{~kg}$ d. Sensitivity calculations have assumed that the local halo density is $0.3 \mathrm{GeV} \mathrm{cm}^{-3}$, the halo circular velocity is $220 \mathrm{~km} \mathrm{~s}^{-1}$, the halo escape velocity is $650 \mathrm{~km} \mathrm{~s}^{-1}$, and that the velocity distribution is Maxwellian. The capabilities of heavy-noble-liquid detectors of the same mass and exposure time should be similar. Over the time scale required to realize the ILC at $1000 \mathrm{GeV}$, we might expect to see a direct detection experiment scaled up to 1 Ton size. This would allow measurement of the annual flux variation and the velocity distribution of directly detected WIMPs. For our analysis, however, we will concentrate on the implication of the total rate measurement combined with collider data.

For the parameters that we have just described, the SuperCDMS group estimates that they will see 16 signal events, assuming a uniform dark matter distribution in the disk and the cross section predicted for LCC1, and negligible background [44]. Dividing the observed rate by the predicted cross section, we would obtain a direct measurement of the flux of dark matter impinging on the detector. We should note that this derived flux is an effective quantity. The detection efficiency for WIMPs depends on the velocity distribution, and the simulation [44] used the efficiency calculated for the reference halo model described in the previous paragraph. In presenting our results, we will refer to the derived quantity as $\Phi_{\text {local }} / \Phi_{0}$, the local flux of WIMPs divided by the flux in the reference model, assuming the same average detection efficiency.

In Fig. 20, we show the likelihood distribution for the effective local flux, obtained by combining the distribution of values of the cross section with the statistical uncertainty of the direct detection measurement. Using the data from the ILC at 1000 $\mathrm{GeV}$, this property of the dark matter halo at the Earth would be measured to $28 \%$ accuracy. 


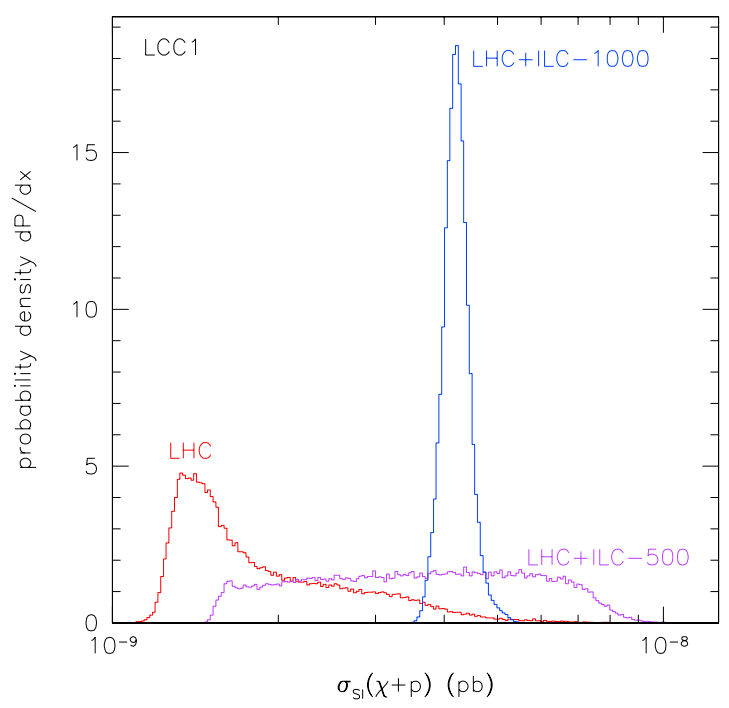

Figure 17: Spin-independent neutralino-proton direct detection cross section for point LCC1. See Fig. 8 for description of histograms.

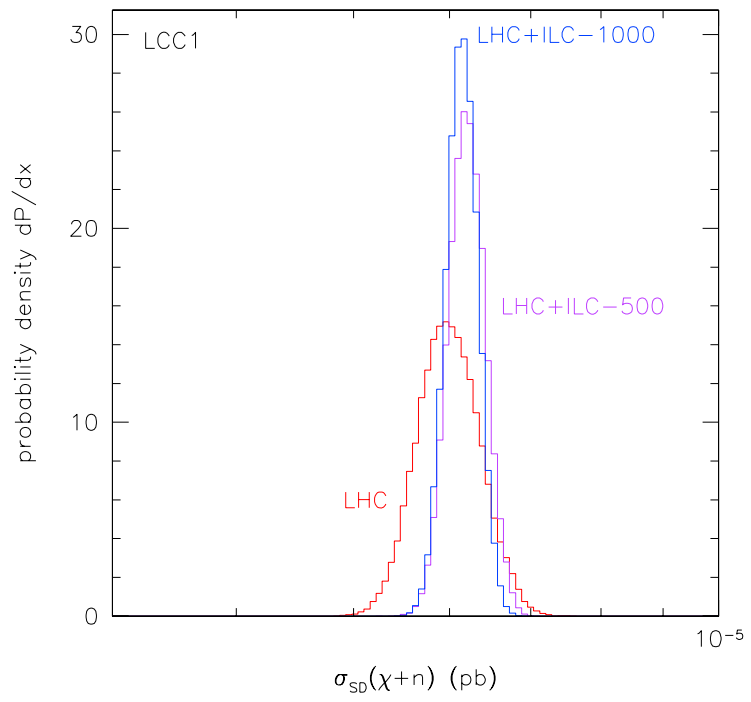

Figure 18: Spin-dependent neutralino-neutron direct detection cross section for point LCC1. See Fig. 8 for description of histograms. 


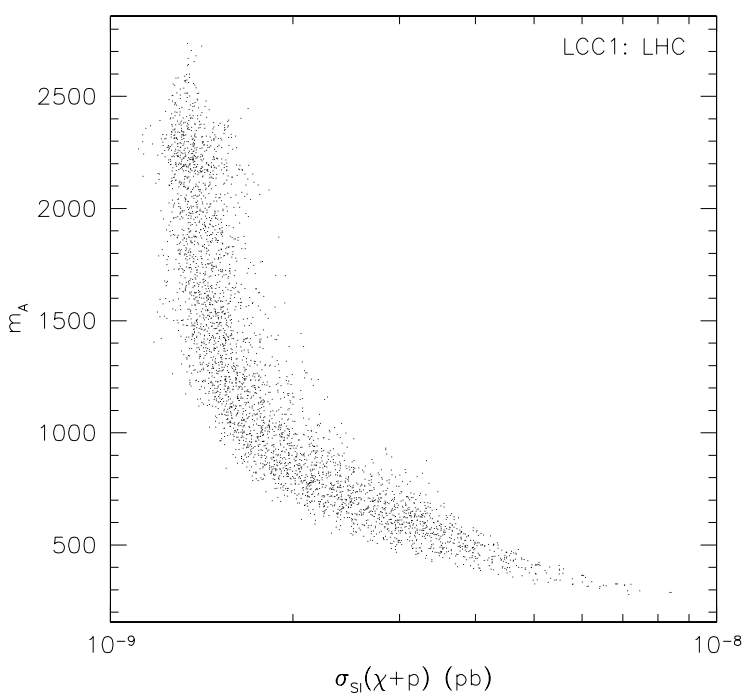

Figure 19: Scatter plot of the spin-independent direct detection cross section vs. $m(A)$ for point LCC1. The strong dependence of the direct detection cross section on the $A^{0}$ mass is clearly seen.

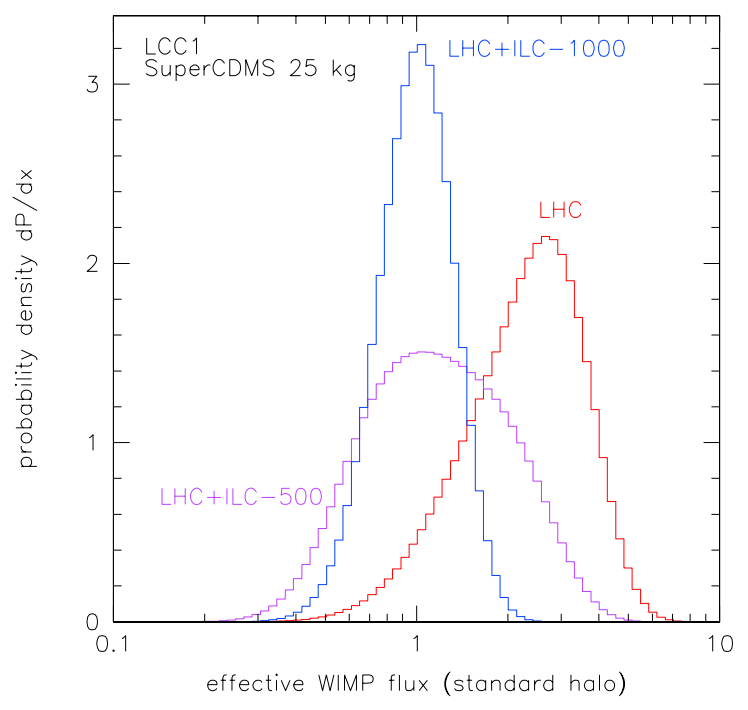

Figure 20: Effective local WIMP flux $\Phi_{\text {local }} / \Phi_{0}$ at the Earth for point LCC1. The results assume the SuperCDMS measurement described in the text. See Fig. 8 for description of histograms. 


\subsection{Constraints from relic density and direct detection}

If LCC1 is the correct theory of Nature, it is possible that, by the end of the decade, the LHC will have observed missing energy events and a convincing signal of dark matter from annihilation to gamma rays will also have been observed. Values of the WIMP mass will have been obtained from the LHC and from the endpoint of the gamma ray spectrum, and these values will have been seen to agree. Underground direct detection experiments in the $25 \mathrm{~kg}$ range such as SuperCDMS may also give the WIMP mass and flux at the Earth. Further, the Planck measurements of the CMB will have provided a very accurate measurement of the cosmic density of dark matter. Under these circumstances, it would be very tempting to use the Planck and SuperCDMS measurements to constrain the parameters of supersymmetry model.

This analysis would depend on very strong assumptions whose status would still be open. It would not be clear that the model leading to missing energy at the LHC was in fact supersymmetry, or that the neutralino was the lightest supersymmetric particle. It would also be unclear whether the neutralino made up $100 \%$ of the dark matter, and whether unknown effects had diluted its abundance. The flux of dark matter particles at the Earth is also uncertain, and depends on the halo model. Nevertheless, by making these assumptions, we could draw very strong conclusions that could later be checked by detailed particle physics measurements.

At this point, LHC data alone provide a prediction of the relic density to $7 \%$ accuracy under the assumptions of the standard cosmology. This is already quite precise. However, the annihilation and direct detection cross sections would not be well determined, and information from a direct dectection experiment could be used to improve our knowledge of the particle physics model. For example, applying a constraint from the direct detection rate to the annihilation cross section improves the determination of this cross section both for the LHC and the ILC-500 data sets. As shown in Fig. 21, the central values of the distributions shift much closer to the correct value, though, curiously, the variances of the distributions are not much improved.

Further into the future, the ILC-500 will predict the relic density at the $1.5 \%$ level. It is thus unlikely that $\mathrm{CMB}$ or other cosmological measurements of the relic density will greatly improve the situation, though if Planck can measure relic density to $0.5 \%$ as advertised, this would be a factor of 3 improvement. The direct detection cross section measurement is now less skewed, with $45 \%$ errors. SuperCDMS would give 16 events, for a $25 \%$ error on the WIMP flux times cross section. In a particular halo model, one might hope to use this measurement, but the advantage would not be likely to be significant.

Fundamentally, the large uncertainty in the direct detection cross section is due 


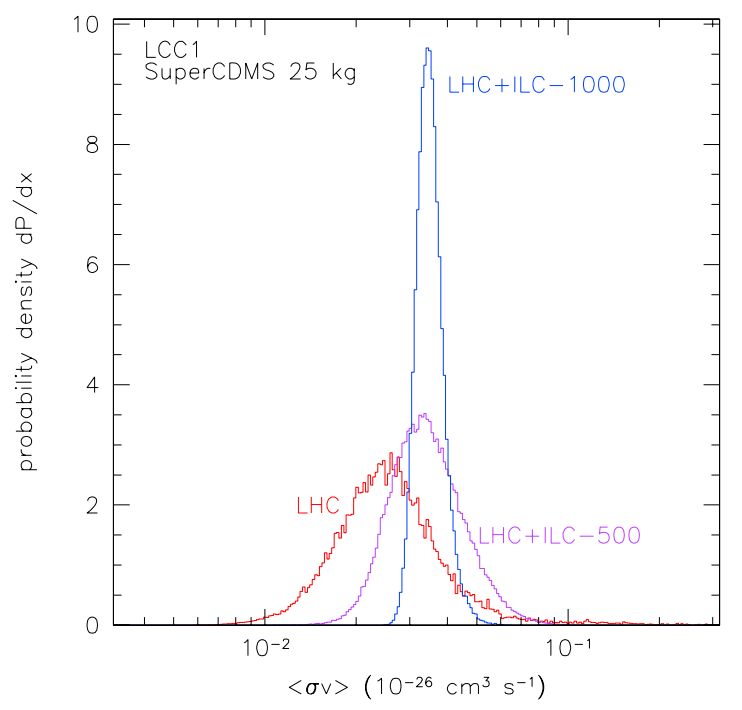

Figure 21: Annihilation cross section at threshold for point LCC1. A direct detection constraint from 2 years of a $25 \mathrm{~kg}$ SuperCDMS is applied. Compared with Fig. 14, it is clear that the direct detection constraint significantly improves the measurement of the annihilation cross section in advance of ILC-1000. See Fig. 8 for description of histograms.

the unknown heavy Higgs mass. Applying the direct detection constraint greatly improves the shape of the distribution of this quantity at LHC and ILC-500, where the heavy Higgs are unobserved, as illustrated in Fig. 22.

The ILC-1000 can predict the relic density at the $0.25 \%$ level and the direct detection cross section at the $5 \%$ level. This is the unique case under study where the astrophysical measurements have no benefit beyond the crucial consistency checks. 

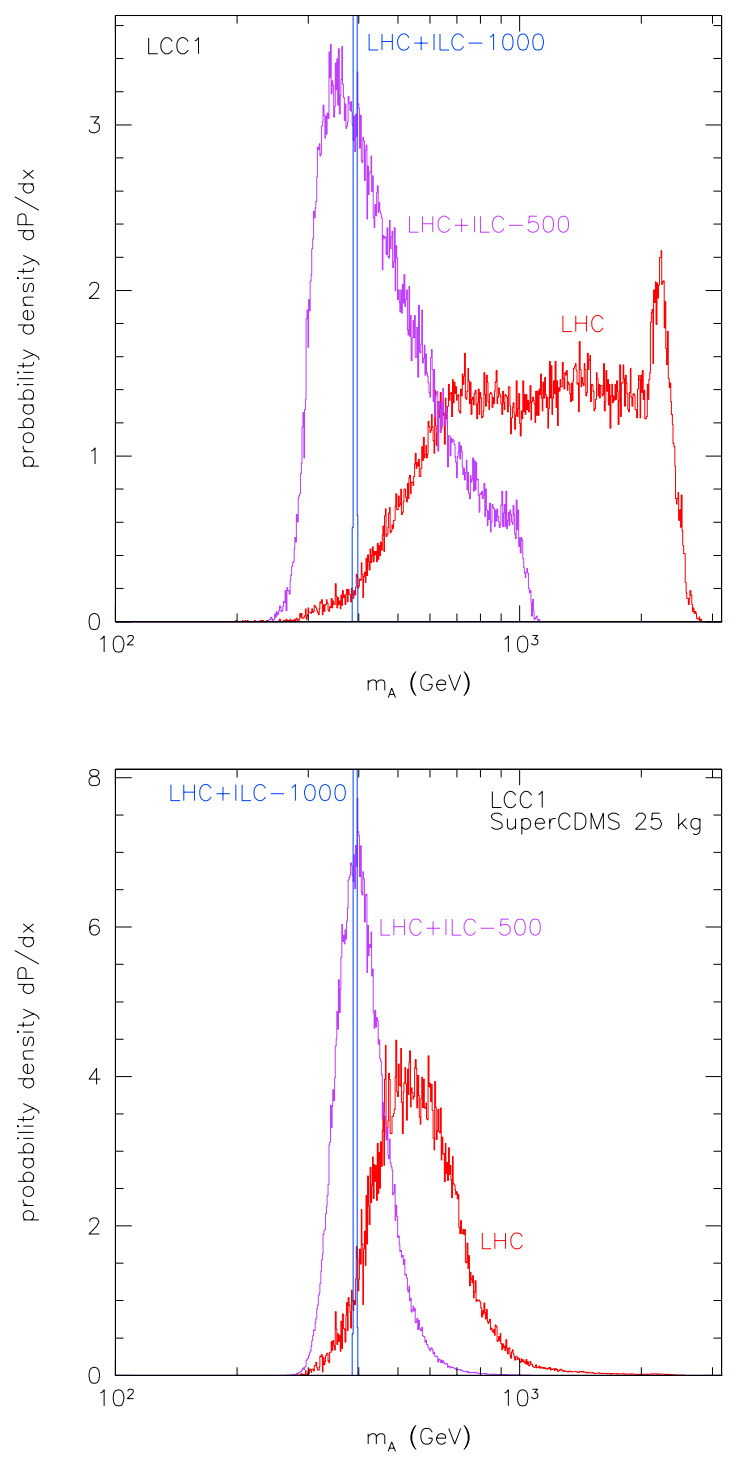

Figure 22: LCC1 heavy Higgs mass $m_{A}$, before and after a direct detection constraint is applied. The constraint allows a crude measurement of the $A^{0}$ mass in advance of the ILC-1000, which directly produces the $A^{0}$. See Fig. 8 for description of histograms. 


\section{Benchmark point LCC2}

In the next few sections, we will carry out the analysis that we have just described at LCC1 for the other three reference points. The parameter set LCC1 was specially chosen by the authors of [63] as a point at which most of particles of the supersymmetry spectrum could be observed at the LHC and then measured precisely at the ILC. In more generic scenarios of supersymmetry, the information available to both colliders will be more limited.

At LCC2, for example, the squarks and sleptons are made extremely heavy, so heavy that it is unlikely that they could be observed at the LHC. However, the model still allows the discovery of supersymmetry at the LHC. The model contains a gluino at $850 \mathrm{GeV}$, giving a cross section of about $10 \mathrm{pb}$ for supersymmetry production at the LHC, and charginos and neutralinos in the range $100-300 \mathrm{GeV}$. The dominant mode of neutralino annihilation is to $W^{+} W^{-}$and $Z^{0} Z^{0}$.

Although we cannot observe the heavy supersymmetric particles, we might hope that the lighter ones, which we can observe, contain most of the information needed to predict the astrophysical cross sections needed to analyze dark matter detection experiments. The main contributions to neutralino annihilation come from diagrams in which neutralinos and charginos are exchanged. The main contribution to the direct detection cross section comes, in this case, from exchange of the light Higgs boson $h^{0}$. Both reactions depend strongly on the gaugino-Higgsino mixing angles, and so the measurement of these angles becomes the major issue for the interpretation of collider measurements in terms of the underlying spectrum parameters.

\subsection{Spectroscopy measurements}

As we have already noted, the LHC will give a large sample of supersymmetry events. Most of these events will involve gluino pair production, followed by gluino decays to $q \bar{q} \chi$, where $\chi$ is a neutralino or chargino. The subsequent evolution can be seen from the spectrum of the model, shown in Fig. 23. The mass difference of the first and third neutralinos is less than $m_{Z}$. Thus, both the second and the third neutralino will decay to the first neutralino through a virtual $Z^{0}$ that can be observed as a lepton pair,

$$
\tilde{\chi}_{i}^{0} \rightarrow \ell^{+} \ell^{-} \tilde{\chi}_{1}^{0}
$$

for $i=2,3$. These processes will allow measurements of the two mass differences to an accuracy comparable to that with which the $\widetilde{\chi}_{2}^{0}-\widetilde{\chi}_{1}^{0}$ mass difference can be measured at LCC1. In addition, the gluino mass should be determined to about $10 \%$ accuracy from the distribution of missing energy and visible transverse mass, and the mass of the lightest neutralino should be determined to about $10 \%$ by more detailed 


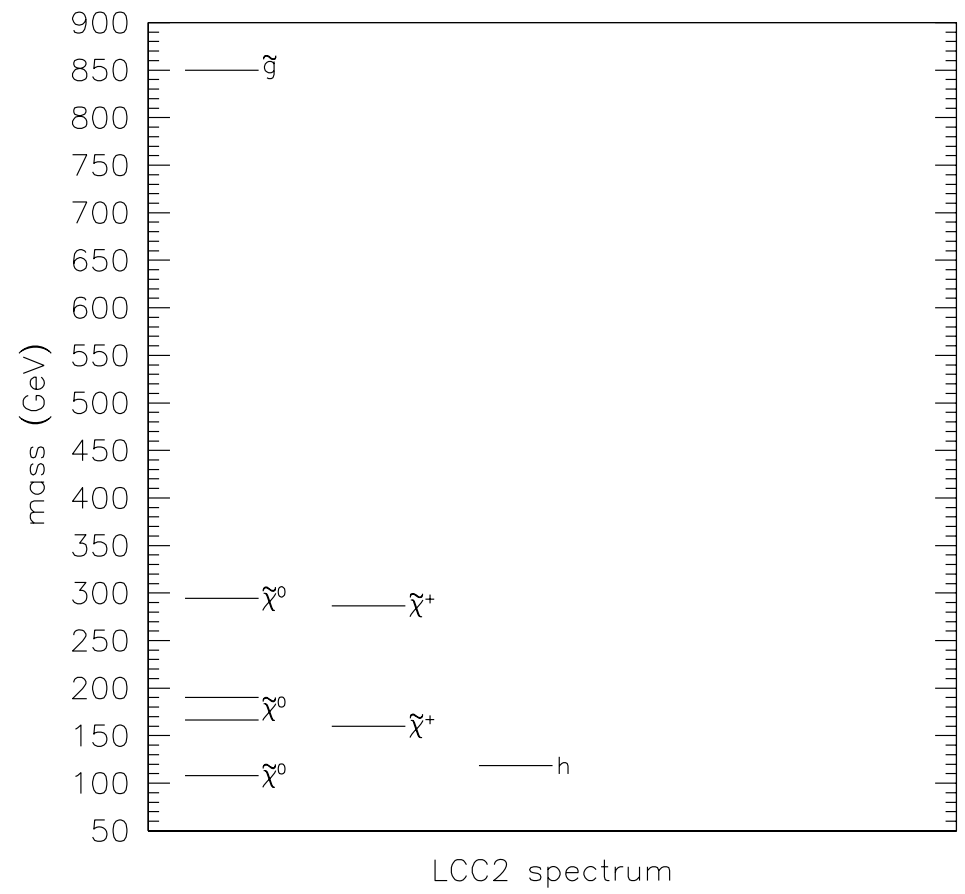

Figure 23: Particle spectrum for point LCC2. All neutralinos and charginos are mixed. The most bino-like neutralino is the lightest one, and the most wino-like neutralino is the heaviest one. All scalars are above about $2 \mathrm{TeV}$. 
kinematic fitting of the these events. Unfortunately, it will be more difficult that at LCC1 to find the charginos or the fourth neutralino, so it is likely that these spectrum parameters are the only ones that can be determined from the LHC data.

Measurements at the ILC will map out much more of the chargino and neutralino spectrum [64]. At a center of mass energy of $500 \mathrm{GeV}$, the ILC will study the lighter states of this spectrum through $e^{+} e^{-} \rightarrow \tilde{\chi}_{1}^{+} \tilde{\chi}_{1}^{-}$and $e^{+} e^{-} \rightarrow \widetilde{\chi}_{2}^{0} \widetilde{\chi}_{3}^{0}$. It will also make a very accurate measurement of the mass of $\tilde{\chi}_{1}^{+}$by determination of the threshold for producing this particle. The $e^{+} e^{-}$production cross sections are sensitive to the gaugino-Higgsino mixing angles and can also be used to constrain the supersymmetry parameters. A second stage of operation at center of mass energies of $1000 \mathrm{GeV}$ can observe the reactions $e^{+} e^{-} \rightarrow \widetilde{\chi}_{3}^{0} \widetilde{\chi}_{4}^{0}$ and $e^{+} e^{-} \rightarrow \widetilde{\chi}_{2}^{+} \widetilde{\chi}_{2}^{-}$and measure the masses of the $\tilde{\chi}_{4}^{0}$ and $\tilde{\chi}_{2}^{+}$.

The complete list of spectrum constraints that we expect for this point for the LHC and for each stage of the ILC is given in Tables 4 and 5.

\subsection{Relic density}

We can now use these constraints on the spectrum as the basis for an exploration of the allowed supersymmetry parameter space. The results of the three Monte Carlo scans, projected onto the axis of the predicted WIMP relic density, are shown in Fig. 24. The distribution from the LHC constraints is quite broad, with a standard deviation of about $40 \%$ and also a significant secondary peak near $\Omega_{\chi} h^{2}=0$. The prediction of $\Omega_{\chi} h^{2}$ from the ILC data a $500 \mathrm{GeV}$ has an accuracy of about $14 \%$, and this improves to about $8 \%$ using the data from the ILC at $1000 \mathrm{GeV}$.

The main difficulty in determining the relic density from the LHC data is that the two precision measurements of mass differences do not provide enough information to fix the gaugino-Higgsino mixing angles. Without an accurate determination of the mixing angles, the relatively accurate determination of the WIMP mass has little predictive power.

However, it is also true that the LHC data can be interpreted in multiple ways in terms of the underlying parameters. This is illustrated by making scatter plots of the Monte Carlo data, as we show in Fig. 25. In Fig. 25(a), we show the scatter plot of the data with LHC constraints in the plane of $m_{1}$ vs. $\mu$. The data clearly shows three solutions, corresponding to a bino-, wino-, and Higgsino-like lightest neutralino. The phase space for the latter two solutions is restricted by our constraint that $m\left(\tilde{\chi}_{1}^{+}\right)$ should be greater than $125 \mathrm{GeV}$. These incorrect solutions are responsible for the peak in the relic density likelihood function at LHC for very small values.

In Fig. 25(b), we show the correlation between $\Omega_{\chi} h^{2}$ and $m(A)$ at the $500 \mathrm{GeV}$ 


\begin{tabular}{|c|c|c|c|c|c|}
\hline mass/mass splitting & LCC2 value & & $\mathrm{LHC}$ & ILC 500 & ILC 1000 \\
\hline$m\left(\widetilde{\chi}_{1}^{0}\right)$ & 107.9 & \pm & 10 & 1.0 & \\
\hline$m\left(\widetilde{\chi}_{2}^{0}\right)-m\left(\tilde{\chi}_{1}^{0}\right)$ & 58.5 & \pm & 1.0 & 0.3 & \\
\hline$m\left(\tilde{\chi}_{3}^{0}\right)-m\left(\tilde{\chi}_{1}^{0}\right)$ & 82.3 & \pm & 1.0 & 0.2 & \\
\hline$m\left(\tilde{\chi}_{4}^{0}\right)-m\left(\tilde{\chi}_{1}^{0}\right)$ & 186.3 & \pm & - & - & 3.0 \\
\hline$m\left(\tilde{\chi}_{1}^{+}\right)$ & 159.7 & \pm & - & 0.55 & \\
\hline$m\left(\tilde{\chi}_{1}^{+}\right)-m\left(\tilde{\chi}_{1}^{0}\right)$ & 51.8 & \pm & - & 0.25 & \\
\hline$m\left(\tilde{\chi}_{2}^{+}\right)$ & 286.7 & \pm & - & - & 1.0 \\
\hline$m\left(\widetilde{e}_{R}\right)$ & 3277. & \pm & $(>350)$ & & $(>480)$ \\
\hline$m\left(\widetilde{\mu}_{R}\right)$ & 3277. & \pm & $(>350)$ & & $(>480)$ \\
\hline$m\left(\widetilde{\tau}_{1}\right)$ & 3252. & \pm & $\left(>m\left(\chi_{2}^{0}\right)\right)$ & & $(>480)$ \\
\hline$m\left(\widetilde{e}_{L}\right)$ & 3280. & \pm & $(>350)$ & & $(>480)$ \\
\hline$m\left(\widetilde{\mu}_{L}\right)$ & 3280. & \pm & $(>350)$ & & $(>480)$ \\
\hline$m\left(\widetilde{\tau}_{2}\right)$ & 3268. & \pm & & & $(>480)$ \\
\hline$m(h)$ & 118.68 & \pm & 0.25 & 0.05 & \\
\hline$m(A)$ & 3242. & \pm & $*$ & $(>240)$ & $(>480)$ \\
\hline$m\left(\widetilde{u}_{R}\right), m\left(\widetilde{d}_{R}\right)$ & 3312. & \pm & $(>2000)$ & & \\
\hline$m\left(\widetilde{s}_{R}\right), m\left(\widetilde{c}_{R}\right)$ & 3312. & \pm & $(>1500)$ & & \\
\hline$m\left(\widetilde{u}_{L}\right), m\left(\widetilde{d}_{L}\right)$ & 3301. & \pm & $(>2000)$ & & \\
\hline$m\left(\widetilde{s}_{L}\right), m\left(\widetilde{c}_{L}\right)$ & 3301. & \pm & $(>1500)$ & & \\
\hline$m\left(\widetilde{b}_{1}\right)$ & 2710 . & \pm & $(>1500)$ & & \\
\hline$m\left(\widetilde{b}_{2}\right)$ & 3241. & \pm & $(>1500)$ & & \\
\hline$m\left(\widetilde{t}_{1}\right)$ & 1976. & \pm & $(>1500)$ & & \\
\hline$m(\widetilde{g})$ & 850. & \pm & 85. & & \\
\hline
\end{tabular}

Table 4: Superparticle masses and their estimated errors or limits for the parameter point LCC2. The notation is as in Table 2.

\begin{tabular}{lccccc} 
cross section & & LCC2 value & & ILC 500 & ILC 1000 \\
\hline \hline$\sigma\left(e^{+} e^{-} \rightarrow \widetilde{\chi}_{1}^{+} \widetilde{\chi}_{1}^{-}\right)$ & LR & $1364 .(0.479)$ & \pm & $1 \%^{*}$ & \\
& RL & $145.6(0.438)$ & \pm & $4 \%^{*}$ & \\
$\sigma\left(e^{+} e^{-} \rightarrow \widetilde{\chi}_{2}^{0} \widetilde{\chi}_{3}^{0}\right)$ & LR & 127.6 & \pm & $4 \%^{*}$ \\
& RL & 105.8 & \pm & $5 \%^{*}$
\end{tabular}

Table 5: SUSY cross sections and estimated errors for the parameter point LCC2. The notation is as in Table 3. The errors labeled by * are taken from the study [64]. 
ILC. Although most of the distribution forms a smooth wide cloud, reflecting the uncertainty in the mixing angles, one sees also a dense corridor at the low end of the allowed range for $m(A)$, leading to vanishingly small relic density. This reflects the influence of the $A^{0}$ pole. At LCC2, the true mass of the $A^{0}$ boson is about $3200 \mathrm{GeV}$; however, we must rely on data to exclude the $A^{0}$ from being in a mass region in which its pole influences the annihilation cross section.

In Fig. 26, we illustrate the power of measuring the polarized neutralino and chargino production cross sections at the ILC. Thje cross sections depend strongly on the gaugino-Higgsino mixing angles; thus, measurement of the cross sections allows us to fix the values of these angles. From the figures, it is clear that there is a significant correlation between these cross sections and the relic density. Furthermore, measuring the cross sections removes the ambiguities in the identities of the particles.

The general form of the three curves in Fig. 24 is, we believe, closer than that of Fig. 8 to the generic situation. The LHC, despite its ability to make precision measurements of the supersymmetry spectrum, has little capability to predict $\Omega_{\chi} h^{2}$. The predictions from the ILC are about at the level of the current determination from WMAP and will not be at the level of the determination from Planck. Nevertheless, the ILC prediction, based only on microscopic data, is quite sufficiently accurate that its agreement or disagreement with the relic density found by Planck would be a striking test of the assertion that the neutralino is the sole source of WIMP dark matter.

\subsection{Annihilation cross section}

In Fig. 27, we show the prediction of our likelihood analysis for the neutralino pair annihilation cross section at threshold. Unlike the situation for LCC1, this figure is very similar in form to the set of predictions for the relic density, with a relatively broad distribution from the LHC constraints and progressively narrower distributions from the two stages of the ILC. This is not so surprising. At LCC2, the dominant annihilation processes that determine the relic density are the decays to $W^{+} W^{-}$and $Z^{0} Z^{0}$. An low energy, these processes go mainly in the S-wave, yielding cross sections that are similar at threshold and at the freeze-out temperature $T / m_{\chi} \sim 1 / 25$. Thus, since the relic density is proportional to the inverse of the annihilation cross section at freeze-out, the likelihood distribution for the relic density should be just the reflection of that for the annihilation cross section. And so it is.

In Fig. 28, we show the likelihood distributions for the exclusive annihilation cross sections to $\gamma \gamma$ and $Z^{0} \gamma$. Unlike the case of LCC1, where the loop diagrams that produce these reactions are dominated by squark exchange, here the dominant effects come from chargino and neutralino exchange. The cross sections thus depend on the 


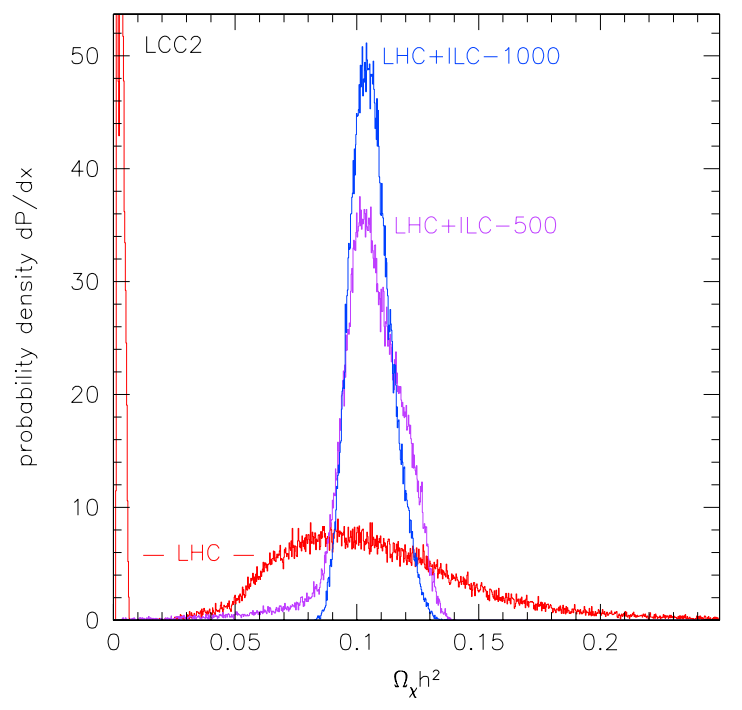

Figure 24: Relic density for point LCC2. There are two overlapping very high peaks at $\Omega_{\chi} h^{2}<0.01$, with maxima at $d P / d x=122$ and 165 , due to the wino and Higgsino solutions to the LHC constraints. See Fig. 8 for description of histograms.

gaugino-Higgsino mixing angles in a way similar to the relic density.

The microscopic determination of the annihilation cross section allows us to interpret observations of gamma rays from dark matter annihilation and to directly measure the density distribution $\langle J(\Omega)\rangle$ for a source of dark matter. In Section 4.4, we described some specific exercises based on the capabilities of GLAST. At LCC2, the annihilation cross section is about 50 times larger, leading to 8,600 signal photons (over 43,000 background) in the GLAST observation of the galactic center and 144 signal photons (over 6 background) in the GLAST observation of the reference subhalo dark matter clump. Folding the photon statistics with the likelihood distributions from Fig. 27, and including a 5\% uncertainty in the background from the galactic center, we find for LCC2 the predictions shown in Fig. 29 for the reconstructed values of $\langle J(\Omega)\rangle$. For the large annihilation cross section characteristic of LCC2, we obtain measurements of $\langle J(\Omega)\rangle$ at the $10 \%$ level. Such measurements would be very powerful constraints on models of dark matter clustering and galaxy formation.

\subsection{Direct detection cross section}

In a similar way, we can repeat the analysis of Section 4.3 for the direct detection cross section. The likelihood distribution of the cross section values given by our 

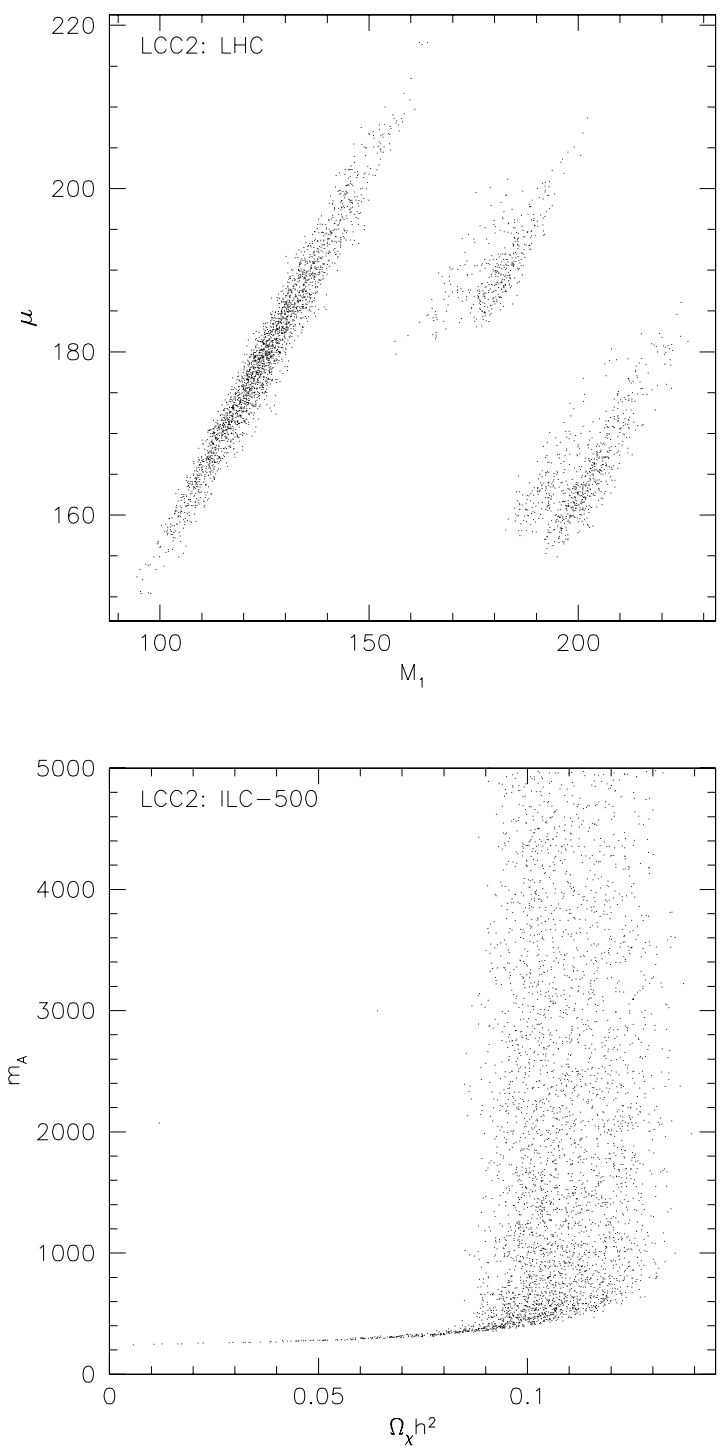

Figure 25: Scatter plots for point LCC2. (a) $m_{1}$ vs. $\mu$ distribution for LHC, illustrating multiple solutions. Left to right, these are bino (correct), wino, and Higgsino. (b) $m_{A}$ vs. $\Omega_{\chi} h^{2}$, showing the influence of resonant annihilation. 

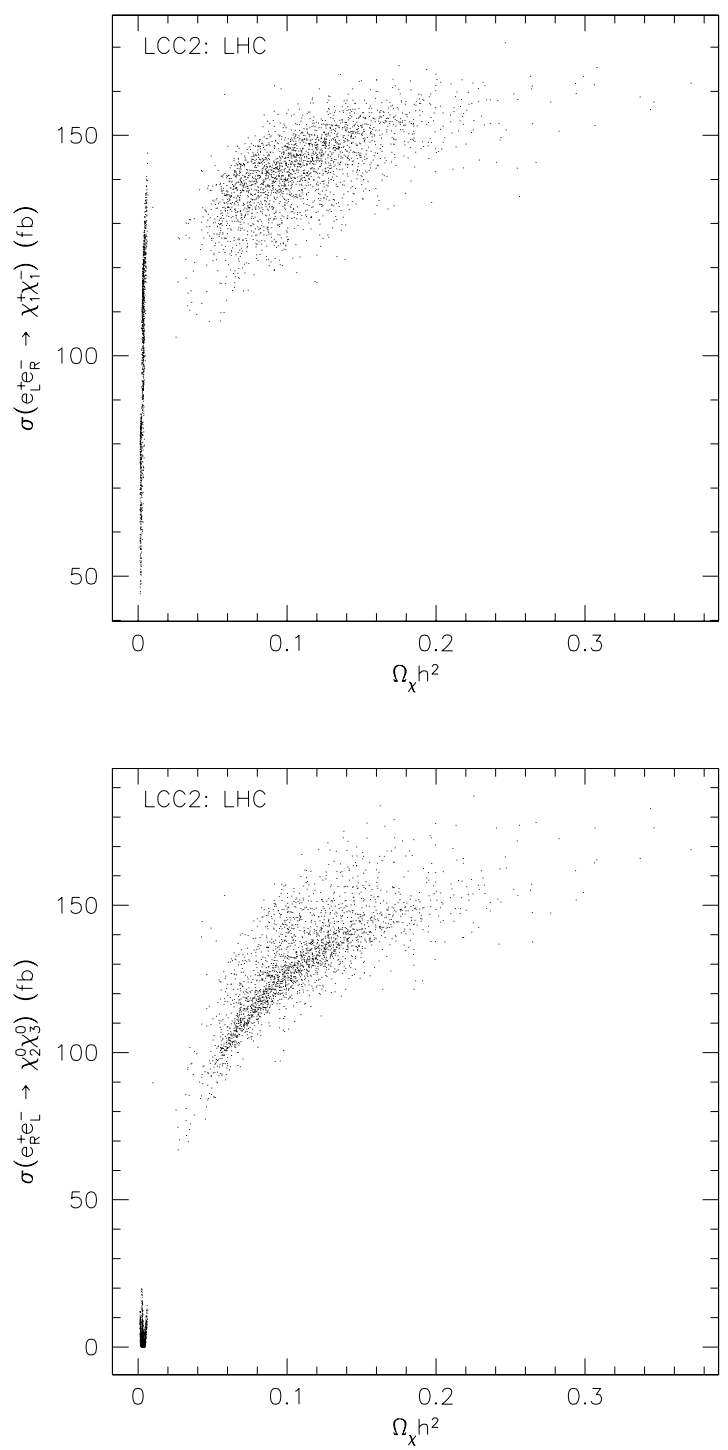

Figure 26: Scatter plots of the LHC data for LCC2 illustrating the effects of $e^{+} e^{-}$cross sections in defining the relic density. The wino and Higgsino islands in Fig. 25(a) are mapped to the lines on the left side of the figures at very low values of $\Omega_{\chi} h^{2}$. Thus they are removed by the $e^{+} e^{-}$cross section constraints. 


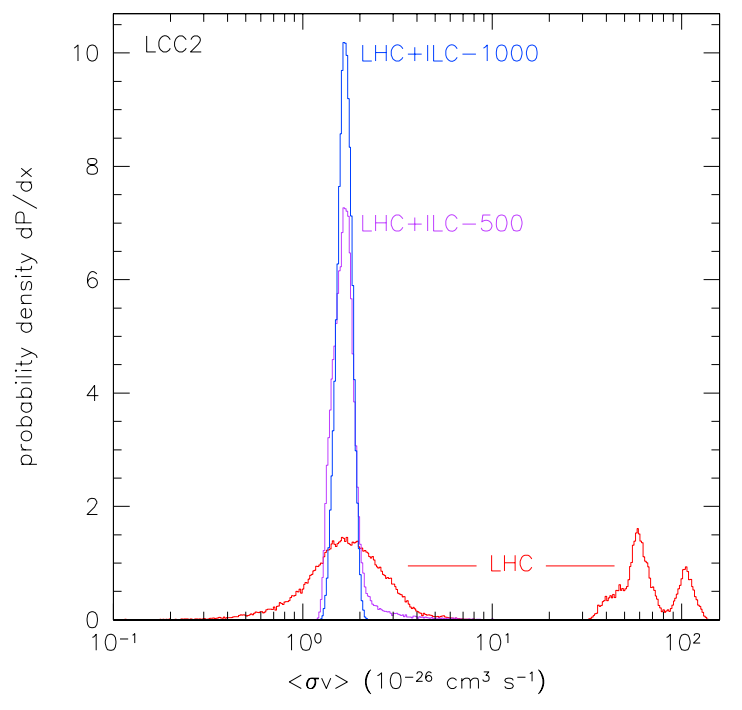

Figure 27: Annihilation cross section at threshold for point LCC2. The wino and Higgsino solutions giving very high cross section are clearly visible. See Fig. 8 for description of histograms.

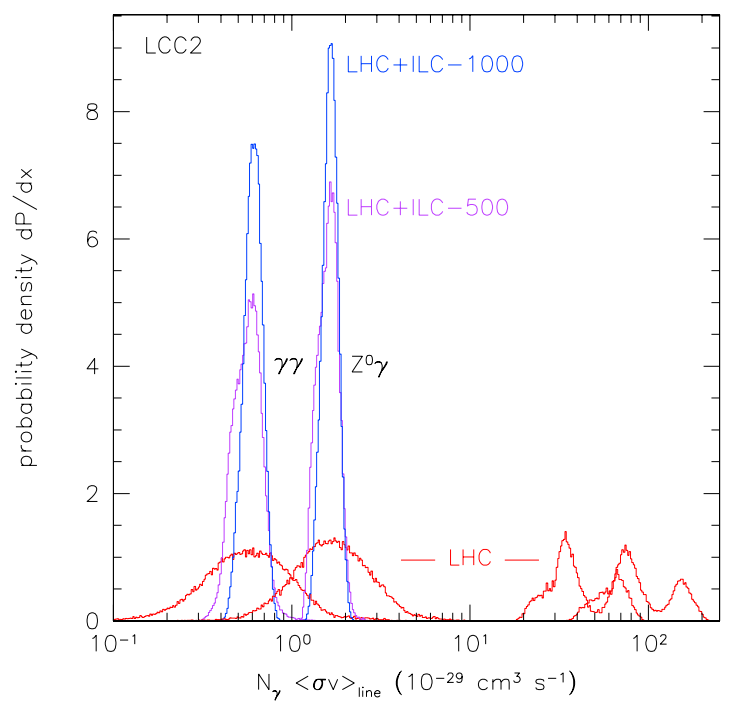

Figure 28: Gamma ray line annihilation cross section at threshold for point LCC2. The wino and Higgsino solutions are clear. See Fig. 8 for description of histograms. 

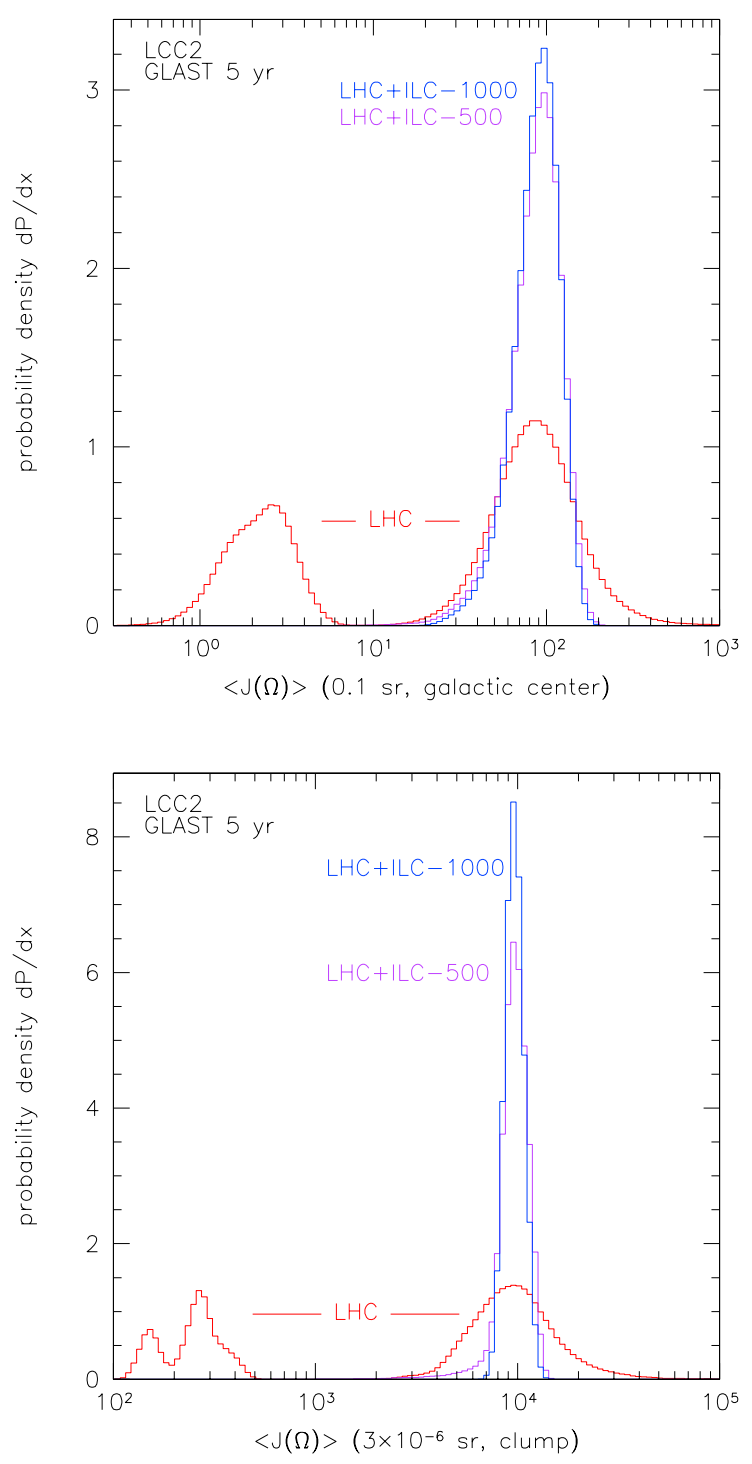

Figure 29: Halo density profiles for point LCC2: (a) galactic center, (b) dark matter clump in the galactic halo. Angle-averaged $J$ values as measured by combining a 5 -year all-sky dataset from GLAST with accelerator measurements are shown. The influence of the incorrect solutions is clearly seen as subsidiary peaks at low $J$ values. See Fig. 8 for description of histograms. 


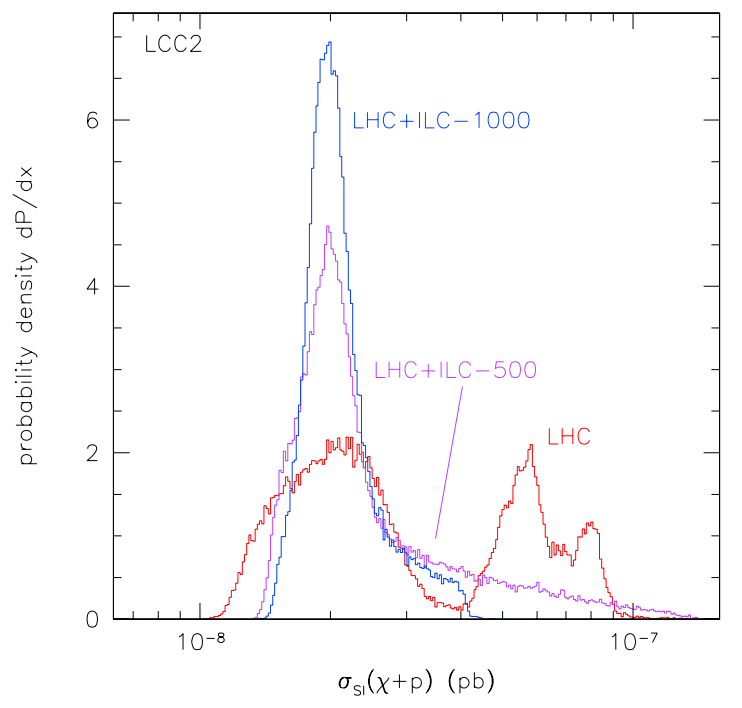

Figure 30: Spin-independent neutralino-proton direct detection cross section for point LCC2. The wino and Higgsino solutions have large cross sections, easily seen. See Fig. 8 for description of histograms.

Monte Carlo scans is shown in Fig. 30. At LCC2, the spin-independent neutralinoproton cross section is dominated by $t$-channel exchange of the light Higgs boson $h^{0}$. The mass of this particle is expected to be measured well at the LHC in the decay $h^{0} \rightarrow \gamma \gamma$. So the LHC constraints already give a reasonably precise estimate of the direct detection cross section, although, as we see from the figure, the ambiguity in the solution leads to additional peaks at high values of the cross section. The ILC measurements sharpen the determination of the mixing angles and also remove the alternative solutions. The corresponding predictions for the spin-dependent part of the neutralino-neutron cross section are shown in Fig 31.

As in Section 4.4, we can combine our microscopic knowledge of the detection cross section with the expected event yield from the SuperCDMS detector to estimate our ability to directly measure the local flux of dark matter at the earth. For this case, we expect a signal of 67 events in SuperCDMS. Folding the statistical uncertainty with the uncertainty in the cross section, we find the determination of the effective local halo flux shown in Fig. 32. 


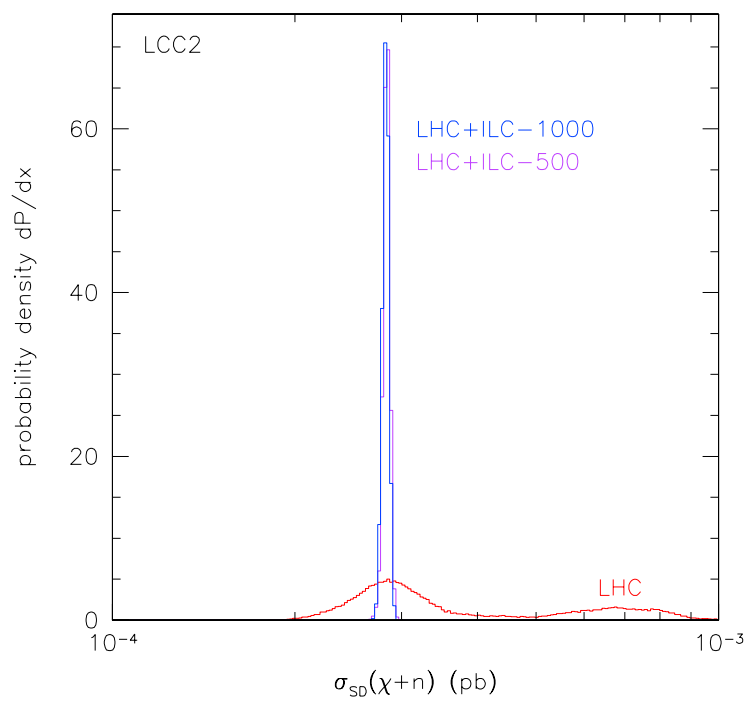

Figure 31: Spin-dependent neutralino-neutron direct detection cross section for point LCC2. See Fig. 8 for description of histograms.

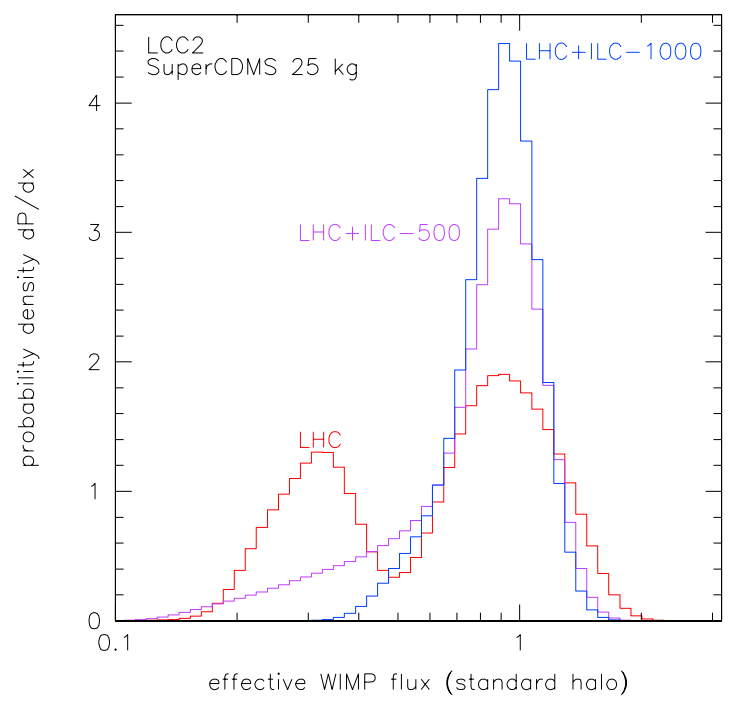

Figure 32: Effective local WIMP flux at the Earth for point LCC2. The results assume the SuperCDMS measurement described in the text. The second peak at low effective flux is due to the high cross section wino and Higgsino solutions. See Fig. 8 for description of histograms. 


\subsection{Constraints from relic density and direct detection}

LCC2 is a special case in that the identity of the lightest neutralino at LHC is unknown, with discrete possibilities: the bino (correct), wino and Higgsino solutions discussed previously. Both incorrect solutions are confined to regions quite far from the central values of both relic density and direct detection cross section. Thus, we find that either the direct detection or relic density constraint completely eliminates the wino and Higgsino solutions for the lightest neutralino. The elimination of these islands has very significant effects on the measurements at LHC, including the annihilation cross section and branching ratios. In fact, the entire structure of the neutralino mass matrix is greatly improved. With either the direct detection or relic density constraint removing the incorrect islands, the bino and wino fractions of every neutralino are measured to better that $15 \%$ at the LHC.

Another unique feature of LCC2 is that the annihilation cross section at $v=0$ is tightly coupled to the relic density. This is because the dominant annihilation channels are to gauge boson pairs, and coannihilations are unimportant. The gauge boson channels are not helicity suppressed, thus the annihilation cross section for typical freeze-out velocities is close to the $v=0$ cross section. At the LHC, any relic density constraint first removes the incorrect solutions which have very large annihilation cross sections, and further constrains the annihilation cross section within the correct solution. The $1 \%$ constraint provides a $4 \%$ measurement of the annihilation cross section. At either stage of the ILC, applying a relic density constraint gives a constraint on annihilation cross section at the same level of precision. At ILC-1000, both of these are measured at the $8 \%$ level, so even here, relic density gives a quite powerful constraint. This tight correlation is illustrated in Fig. 33.

A third unique feature of LCC2 is that the dominant process in direct detection is the exchange of the light Higgs boson, because the heavy Higgses are very heavy, at 3.2 TeV. Because the lightest neutralino is mixed, the cross section is quite large. If the heavy Higgs were lighter, the cross section would be even larger. Using the direct detection constraint thus places a lower limit of $1 \mathrm{TeV}$ on the mass of the heavy Higgs boson, which is much stronger that any direct lower limit that can be obtained from LHC or ILC.

\section{Benchmark point LCC3}

In Sections 6 and 7 we turn to points illustrating special circumstances in which the neutralino relic density depends on accidental relationships among particle masses in the theory. At LCC1, we would obtain the correct neutralino relic density if sleptons 


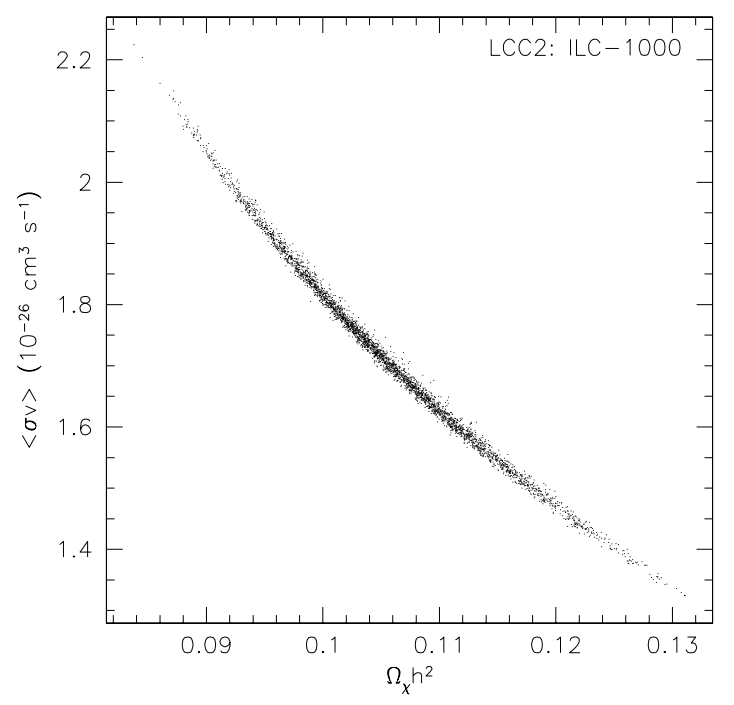

Figure 33: Scatter plot of annihilation cross section against relic density for point LCC2. Given ILC-1000 data, the two quantities are very well correlated.

exchanged in the $t$-channel would light enough; at LCC2, would obtain the correct neutralino relic density if the gaugino-Higgsino mixing angles were large enough. These are generic constraints valid in large regions of the MSSM parameter space. At LCC3 and LCC4, we require more specific tuning of particle masses against one another.

The essential physics of the neutralino relic density at LCC3 is coannihilation of the neutralino with the stau lepton. That is, the dominant annihilation reactions that determine the relic density are $\widetilde{\tau} \chi_{1}^{0} \rightarrow \tau \gamma$ and $\widetilde{\tau} \widetilde{\tau} \rightarrow \tau \tau$, both of which can proceed in the S-wave. The relative density of $\widetilde{\tau}$ particles relative to neutralinos during the annihilation process is [91]

$$
\exp \left[-\frac{m(\tilde{\tau})-m(\chi)}{T}\right] \approx \exp \left[-25\left(\frac{m(\widetilde{\tau})-m(\chi)}{m(\chi)}\right)\right] .
$$

Thus, this mechanism works only a a narrow region of parameter space, one that becomes increasingly constrained as the mass of the neutralino increases.

\subsection{Spectroscopy measurements}

The supersymmetry spectrum at the point LCC3 is shown in Fig. 34. The point is one at which the LHC will be able to obtain a reasonably complete overview of 


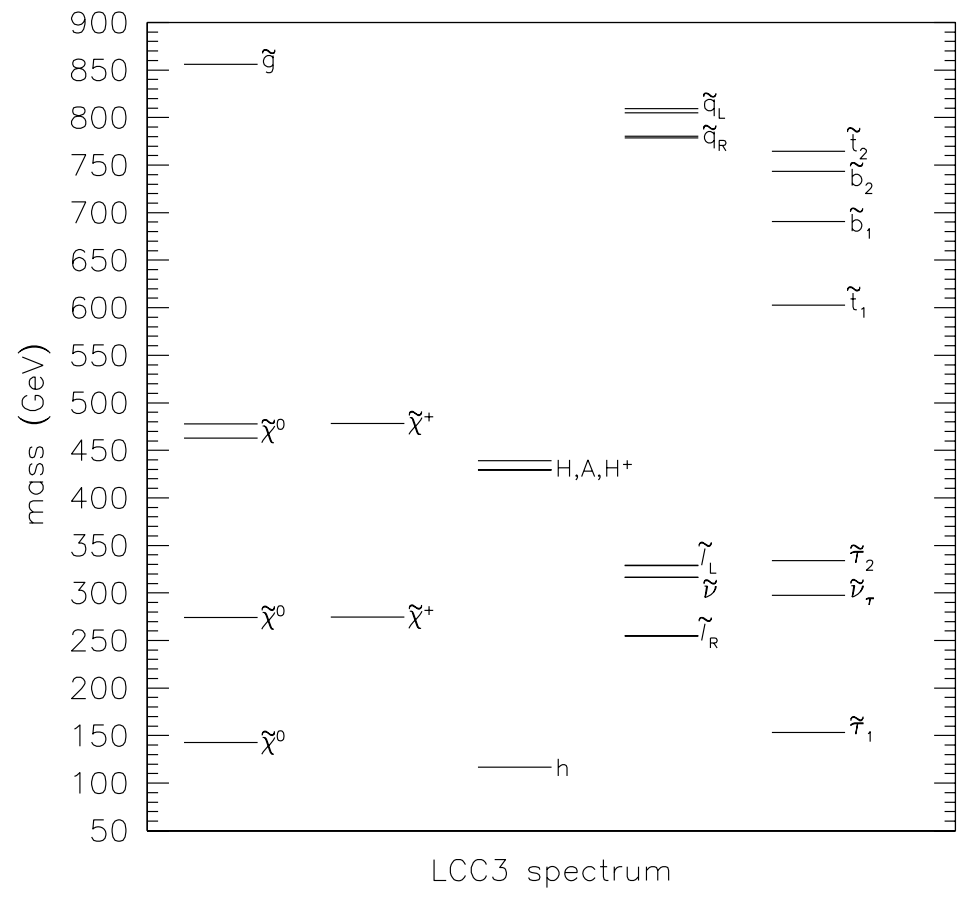

Figure 34: Particle spectrum for point LCC3. The stau-neutralino mass splitting is 10.8 $\mathrm{GeV}$. The lightest neutralino is predominantly bino, the second neutralino and light chargino are predominantly wino, and the heavy neutralinos and chargino are predominantly Higgsino. 
the supersymmetry spectrum. The squarks and the gluino have masses of about 800 $\mathrm{GeV}$, leading to cross sections of tens of pb for supersymmetry production. Effects enhanced by the large value of $\tan \beta$ move the lighter top and bottom squarks down to about $600 \mathrm{GeV}$. The $H^{0}$ and $A^{0}$ bosons can be observed in their decay to $\tau^{+} \tau^{-}$.

Unfortunately, we expect that the very precise spectrum measurements that would be available at LCC1 and LCC2 will not be possible here. The dominant decay mode of the $\widetilde{\chi}_{2}^{0}$ is $\widetilde{\chi}_{2}^{0} \rightarrow h^{0} \widetilde{\chi}_{1}^{0}$. The right-handed sleptons are lighter than the $\widetilde{\chi}_{2}^{0}$, so that the decay chains $\widetilde{\chi}_{2}^{0} \rightarrow \ell \widetilde{\ell} \rightarrow \ell^{+} \ell^{-} \widetilde{\chi}_{1}^{0}$ are kinematically allowed. However, for the case of $\widetilde{e}_{R}$ and $\widetilde{\mu}_{R}$, the branching fractions of $\widetilde{\chi}_{2}^{0}$ to these channels are only $2 \times 10^{-5}$, giving too small an event sample to be useful. For the decay chain involving $\widetilde{\tau}_{1}$, the branching fraction is $4 \%$, but the mass splitting between the $\widetilde{\tau}_{1}$ and the $\widetilde{\chi}_{1}^{0}$ is only 10 $\mathrm{GeV}$. This small mass gap is chosen precisely so that the coannihilation channels will dominate in the computation of the relic density. At the LHC, this means that the $\tau$ from $\widetilde{\tau}_{1}$ decay will be too soft to be observed, and so the kinematic constraints from the lepton cascade cannot be used. In particular, this means that the most important spectrum parameter in the coannihilation region- the $\widetilde{\tau}_{1}-\widetilde{\chi}_{1}^{0}$ mass splitting - cannot be measured at the LHC. In our study, we have assumed that the mass of the $\widetilde{\chi}_{1}^{0}$ can be obtained to $10 \%$ accuracy and the mass of the $\tilde{\chi}_{2}^{0}$ to $15 \%$ accuracy by general kinematic fitting of supersymmetry events, and that the masses of the $\widetilde{e}_{R}$ and $\widetilde{\mu}_{R}$ can be found to $20 \%$ accuracy using slepton pair production.

At the ILC, the studies $[65,72]$ have shown that it is possible to observe the stau decay to tau and to measure the mass splitting. The study [65] obtains an error on the mass splitting of about $1 \mathrm{GeV}$ from kinematic fitting of the $e^{+} e^{-} \rightarrow \widetilde{\tau}^{+} \widetilde{\tau}^{-}$events. The study [72] obtains an error of about $0.5 \mathrm{GeV}$ on the stau mass from a scan of the $\widetilde{\tau}^{+} \widetilde{\tau}^{-}$ threshold. This must be combined with a precision measurement of the neutralino mass, which can be obtained from the analysis of the reaction $e^{+} e^{-} \rightarrow \widetilde{\chi}_{1}^{0} \widetilde{\chi}_{2}^{0}$. The value of the cross section for $e^{+} e^{-} \rightarrow \widetilde{\tau}^{+} \widetilde{\tau}^{-}$events measures the stau mixing angle.

However, this is the extent of the information that can be found at this point at the $500 \mathrm{GeV}$ ILC. To fix the gaugino-Higgsino mixing angles and $\tan \beta$, more information is needed. These additional constraints can be provided by the $1000 \mathrm{GeV}$ ILC, from two sources. First, the remaining states of the chargino and neutralino system can be discovered and studied in the reactions $e^{+} e^{-} \rightarrow \widetilde{\chi}_{3}^{0} \widetilde{\chi}_{4}^{0}$ and $e^{+} e^{-} \rightarrow \widetilde{\chi}_{1}^{+} \widetilde{\chi}_{2}^{-}$. Second, because the mass of the $A^{0}$ Higgs boson at LCC3 is $428 \mathrm{GeV}$, this particle can be observed in the process $e^{+} e^{-} \rightarrow H^{0} A^{0}$. The mass of the $A^{0}$ can be important for the detection cross sections, as we have seen, but also the total width of the $A^{0}$ is interesting as a way to measure $\tan \beta$. We have estimated the error on the $\Gamma_{A}$ following [67] and used this as a constraint.

The complete list of spectrum constraints that we expect for this point for the LHC and for each stage of the ILC is given in Tables 6 and 7. 


\begin{tabular}{|c|c|c|c|c|c|}
\hline mass/mass splitting & LCC3 value & & $\mathrm{LHC}$ & ILC 500 & ILC 1000 \\
\hline$m\left(\widetilde{\chi}_{1}^{0}\right)$ & 142.6 & \pm & 14. & 0.1 & \\
\hline$m\left(\widetilde{\chi}_{2}^{0}\right)$ & 274.2 & \pm & 41. & & \\
\hline$m\left(\tilde{\chi}_{2}^{0}\right)-m\left(\tilde{\chi}_{1}^{0}\right)$ & 131.5 & \pm & - & 0.5 & \\
\hline$m\left(\widetilde{\chi}_{3}^{0}\right)-m\left(\widetilde{\chi}_{1}^{0}\right)$ & 320.2 & \pm & - & - & 2.0 \\
\hline$m\left(\tilde{\chi}_{4}^{0}\right)-m\left(\tilde{\chi}_{1}^{0}\right)$ & 335.4 & \pm & - & - & 2.0 \\
\hline$m\left(\widetilde{\chi}_{1}^{+}\right)$ & 274.5 & \pm & - & - & 0.7 \\
\hline$m\left(\tilde{\chi}_{2}^{+}\right)$ & 478.2 & \pm & - & - & 2.0 \\
\hline$m\left(\widetilde{e}_{R}\right)$ & 254.9 & \pm & $50 .^{a}$ & - & 1.0 \\
\hline$m\left(\widetilde{\mu}_{R}\right)$ & 254.7 & \pm & $50 .^{b}$ & & \\
\hline$m\left(\widetilde{e}_{R}\right)-m\left(\tilde{\chi}_{1}^{0}\right)$ & 112.3 & \pm & - & - & 0.2 \\
\hline$m\left(\widetilde{\mu}_{R}\right)-m\left(\widetilde{\chi}_{1}^{0}\right)$ & 112.1 & \pm & - & - & 0.2 \\
\hline$m\left(\widetilde{\tau}_{1}\right)$ & 153.4 & \pm & - & 0.5 & \\
\hline$m\left(\widetilde{\tau}_{1}\right)-m\left(\tilde{\chi}_{1}^{0}\right)$ & 10.8 & \pm & - & 1.0 & \\
\hline$m\left(\widetilde{e}_{L}\right)$ & 328.9 & \pm & $@^{a}$ & & \\
\hline$m\left(\widetilde{\mu}_{L}\right)$ & 329.1 & \pm & $@^{b}$ & & \\
\hline$m\left(\widetilde{e}_{L}\right)-m\left(\tilde{\chi}_{0}^{1}\right)$ & 186.3 & \pm & - & - & 1.0 \\
\hline$m\left(\widetilde{\mu}_{L}\right)-m\left(\tilde{\chi}_{0}^{1}\right)$ & 186.5 & \pm & - & - & 1.0 \\
\hline$m\left(\widetilde{\tau}_{2}\right)-m\left(\widetilde{\chi}_{0}^{1}\right)$ & 191.3 & \pm & - & - & 3.0 \\
\hline$m(h)$ & 116.58 & \pm & 0.25 & 0.05 & \\
\hline$m(A)$ & 429.5 & \pm & $1.5 *$ & & 0.8 \\
\hline$\Gamma(A)$ & 9.1 & \pm & & & 1.0 \\
\hline$m\left(\widetilde{u}_{R}\right), m\left(\widetilde{d}_{R}\right)$ & $780 ., 778$. & \pm & 78. & & \\
\hline$m\left(\widetilde{s}_{R}\right), m\left(\widetilde{c}_{R}\right)$ & $778 ., 780$. & \pm & 78 . & & \\
\hline$m\left(\widetilde{u}_{L}\right), m\left(\widetilde{d}_{L}\right)$ & $805 ., 809$. & \pm & 121. & & \\
\hline$m\left(\widetilde{\sim}_{L}\right), m\left(\widetilde{c}_{L}\right)$ & 809., 805. & \pm & 121. & & \\
\hline$m\left(\widetilde{b}_{1}\right)$ & 690. & \pm & 35 . & & \\
\hline$m\left(\widetilde{b}_{2}\right)$ & 743. & \pm & 74. & & \\
\hline$m\left(\widetilde{t}_{1}\right)$ & 603. & \pm & $(>315)$ & & \\
\hline$m(\widetilde{g})$ & 856. & \pm & 171. & & \\
\hline
\end{tabular}

Table 6: Superparticle masses and their estimated errors or limits for the parameter point LCC3. The notation is as in Table 2. The LHC measurements of slepton masses apply to the lighter of $\widetilde{e}_{R}, \widetilde{e}_{L}$ and the lighter of $\widetilde{\mu}_{R}, \widetilde{\mu}_{L}$. The symbol '*' indicates that, because the $A^{0}$ can be seen at LHC, $\tan \beta>7\left(m_{A} / 200\right)$. 


\begin{tabular}{|c|c|c|c|c|c|}
\hline cross section & & LCC3 value & & ILC 500 & ILC 1000 \\
\hline minimal set & & & & & \\
\hline $\bar{\sigma}\left(e^{+} e^{-} \rightarrow \widetilde{\chi}_{1}^{0} \widetilde{\chi}_{2}^{0}\right)$ & LR & 34.4 & \pm & $8 \% *$ & \\
\hline & RL & 2.1 & \pm & - & \\
\hline$\sigma\left(e^{+} e^{-} \rightarrow \widetilde{\tau}_{1}^{+} \widetilde{\tau}_{1}^{-}\right)$ & LR & 45.6 & \pm & $100 \% *$ & \\
\hline & RL & 103.4 & \pm & $4 \%^{*}$ & \\
\hline$\sigma\left(e^{+} e^{-} \rightarrow \tilde{\chi}_{1}^{+} \tilde{\chi}_{1}^{-}\right)$ & LR & $212.3(0.808)$ & \pm & & $3 \%$ \\
\hline & RL & $6.3(0.774)$ & \pm & & - \\
\hline$\sigma\left(e^{+} e^{-} \rightarrow \tilde{\chi}_{2}^{0} \tilde{\chi}_{2}^{0}\right)$ & LR & 88.7 & \pm & & $5 \%$ \\
\hline & RL & 2.5 & \pm & & - \\
\hline$\sigma\left(e^{+} e^{-} \rightarrow \widetilde{e}_{R}^{+} \widetilde{e}_{R}^{-}\right)$ & $\mathrm{LR}$ & $19.5(0.735)$ & \pm & $10 \%$ & \\
\hline & $\mathrm{RL}$ & $350.5(0.971)$ & \pm & $3 \%$ & \\
\hline
\end{tabular}

Table 7: SUSY cross sections and estimated errors for the parameter point LCC3. The notation is as in Table 3. The symbol (-) denotes that the cross section is less than $10 \mathrm{fb}$ or is otherwise not measurable. The errors labeled by * are taken from the study $[65,66]$; the others are estimated using (11).

\subsection{Relic density}

We can use these constraints on the spectrum as the basis for an exploration of the allowed supersymmetry parameter space. The results from the three Monte Carlo scans, projected onto the axis of the predicted WIMP relic density, are shown in Fig. 35. Because the LHC data is not sensitive to the stau-neutralino mass difference, that set of constraints leads to essentially no information about the relic density, yielding a distribution that stretches well to the right of the region plotted. The information from the $500 \mathrm{GeV}$ ILC can only check that we are in the stau coannihilation region, predicting the relic density only within a factor of two. However, when the information from the $1000 \mathrm{GeV}$ ILC is added, with strong constraints on the mixing angles and on $\tan \beta$, the relic density is predicted to about $18 \%$, an error three times as large as the current WMAP determination.

Though this is not the main issue for the LHC, the scan data does show quite clearly the presence of continuous ambiguities in the interpretation of the spectrum data in terms of underlying parameters. In Fig. 36, we show the correlation of the parameters $m_{1}$ and $\mu$. The plot shows three regions, two horizontal and one vertical, corresponding to models in which the lightest neutralino is mainly bino, wino, and Higgsino, respectively.

The dependence of the coannihilation cross sections on parameters that are not fixed at the $500 \mathrm{GeV}$ ILC is illustrated by the scatter plot shown in Fig. 37. This plot shows the correlation between $\Omega_{\chi} h^{2}, \tan \beta$, and $\Gamma_{A}$. The relic density is mainly 


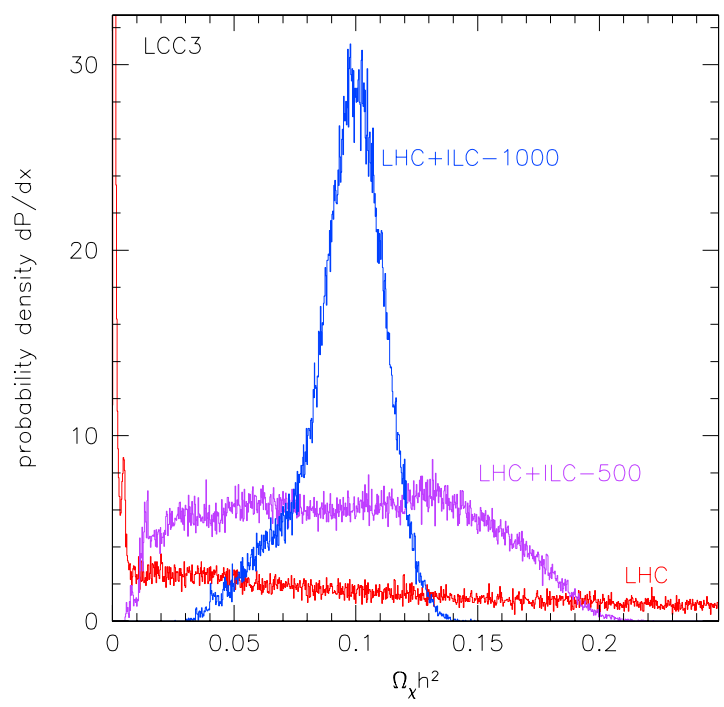

Figure 35: Relic density measurement for point LCC3. The wino peak at very small relic density is clear. See Fig. 8 for description of histograms.

determined by annihilations of the stau. This plot makes clear how the measurement of $\Gamma_{A}$, which fixes $\tan \beta$ to about \pm 2 , has such an important effect. At the ILC-1000, there remains a weak correlation between the stau-neutralino mass splitting and relic density, illustrated in Fig. 38.

\subsection{Annihilation cross section}

In Fig. 39, we show the prediction of our likelihood analysis for the neutralino pair annihilation cross section at threshold. The dominant annihilation processes contributing to the relic density at LCC3 are actually coannihilation reactions, and these are not longer available, because all primordial staus have decayed long ago. So we have a situation similar to that of LCC1, in which a subdominant annihilation reaction for the relic density becomes the most important one for the threshold cross section. The relevant reaction is the same one that was important at LCC1, $\chi \chi \rightarrow b \bar{b}$, by $t$-channel $\widetilde{b}$ exchange, but also getting a contribution from the $A^{0} s$-channel resonance. The relative influence of these two contributions is reflected in the sharpening of the distribution after the $A^{0}$ is determined at the $1000 \mathrm{GeV}$ ILC. In the LHC distribution, we again see a subsidiary peak at high values of the cross section that reflects the possibility of solutions in which the lightest neutralino is wino- or Higgsino-like. In Fig. 40, we show the similar evolution for the exclusive annihilation cross sections to $\gamma \gamma$ and $\gamma Z$. 


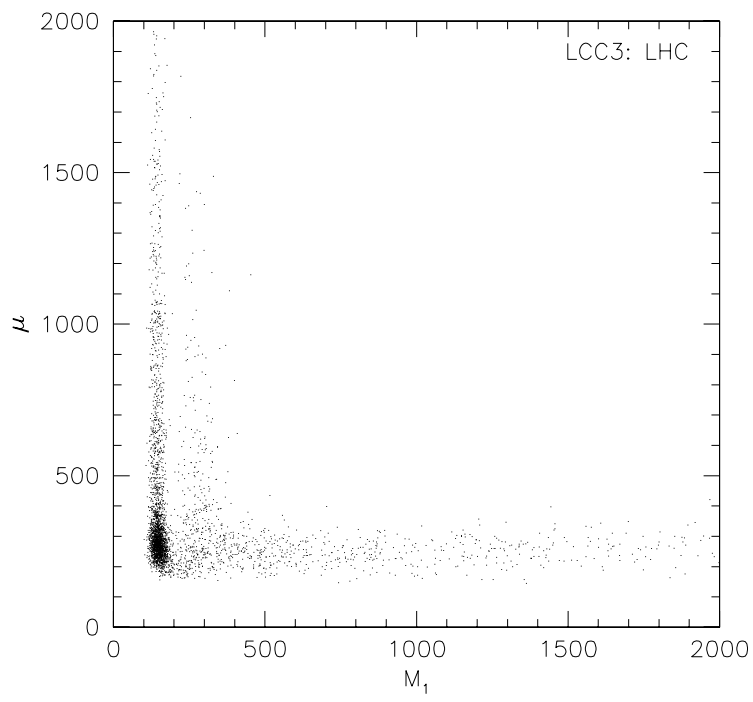

Figure 36: Scatter plot of $\mu$ vs. $m_{1}$ for LHC data at point LCC3. The "F" structure indicates the fact that the lightest neutralino is either bino or wino, while the second could be anything.

In Fig 41, we show the result of carrying out the exercise described in Section 4.4 in which we combine collider data with the annihilation gamma ray signal that should be found by GLAST. For the galactic center, we expect 2,100 signal photons, over 43,000 background; for the canonical halo object, we expect 35 signal photons, over 6 background. Using these counting rates and the determination of the annihilation cross section that we have described, we obtain the predictions for $\langle J(\Omega)\rangle$ shown in Fig. 41. The quality of the determinations is somewhat better than in the case of LCC1, For the halo object, we already obtain a $20 \%$ measurement with this relatively small signal.

\subsection{Direct detection cross section}

At LCC3, we return to the situation seen at LCC1 in which the direct detection cross section is dominated by the $t$-channel exchange of the heavy Higgs boson $H^{0}$. The direct detection cross section is poorly determined by the LHC data, even though the mass of the $H^{0}$ is known. However, the progressive clarification of the supersymmetry mixing angles and the value of $\tan \beta$ at the $500 \mathrm{GeV}$ and $1000 \mathrm{GeV}$ ILC yields a fairly precise determination. The evolution is shown in Fig. 42. The corresponding predictions for the spin-dependent part of the neutralino-neutron cross section are shown in Fig 43. 

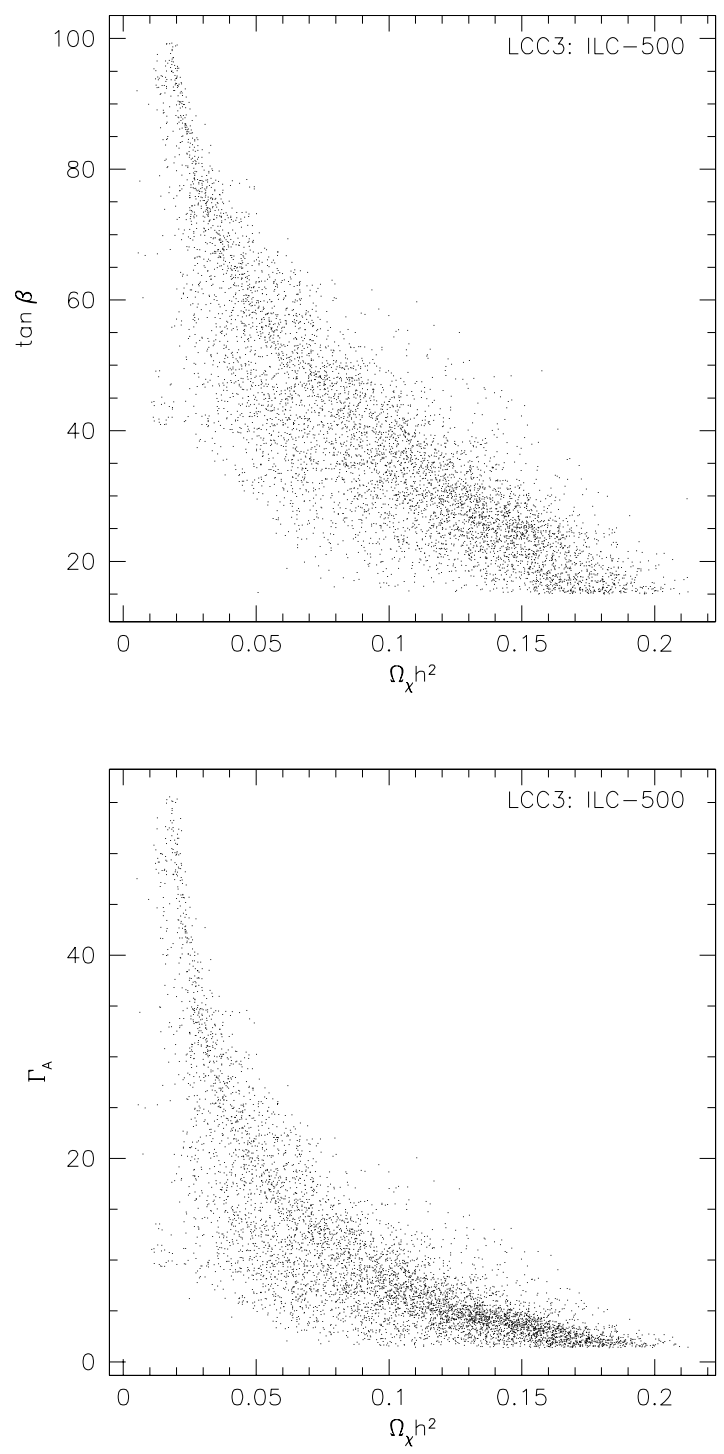

Figure 37: Scatter plots of both $\tan \beta$ and $\Gamma_{A}$ vs. relic density for point LCC3. There is a significant correlation, which can be resolved at ILC-1000. In fact, the correlation between $\tan \beta$ and $\Gamma_{A}$ is quite close. 


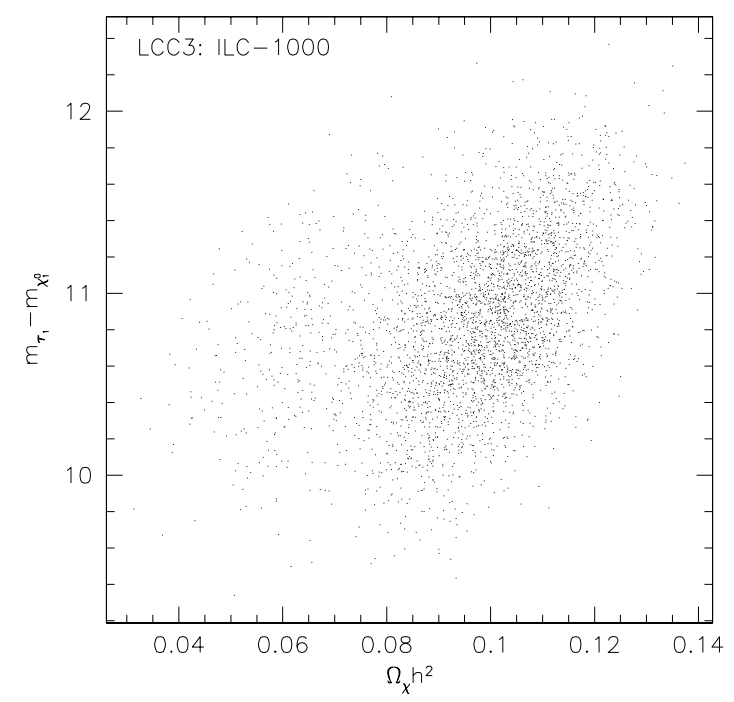

Figure 38: Scatter plot of $m_{\tilde{\tau}}-m_{\chi_{1}^{0}}$ for point LCC3, ILC-1000 sample. A correlation exists, but it is not strong.

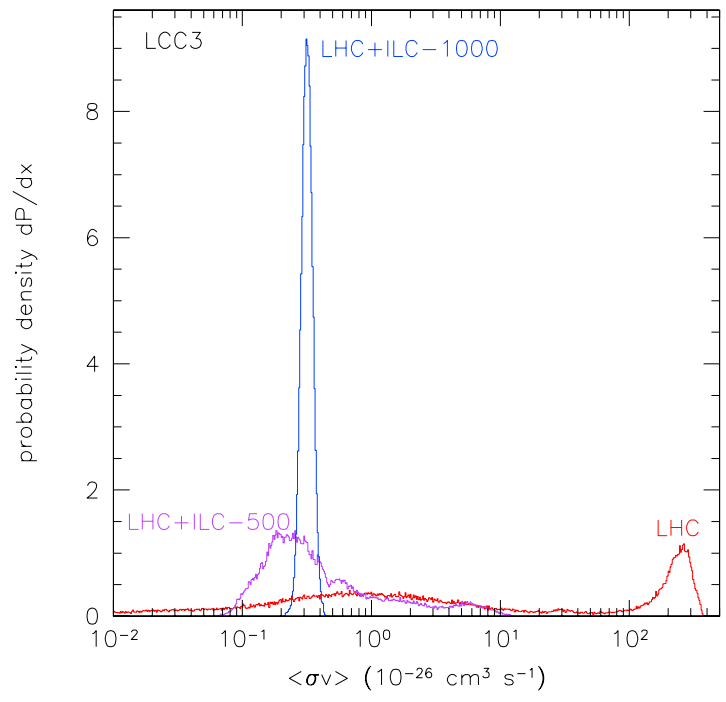

Figure 39: Annihilation cross section at threshold for point LCC3. The wino solution giving very high cross section is clearly visible. See Fig. 8 for description of histograms. 


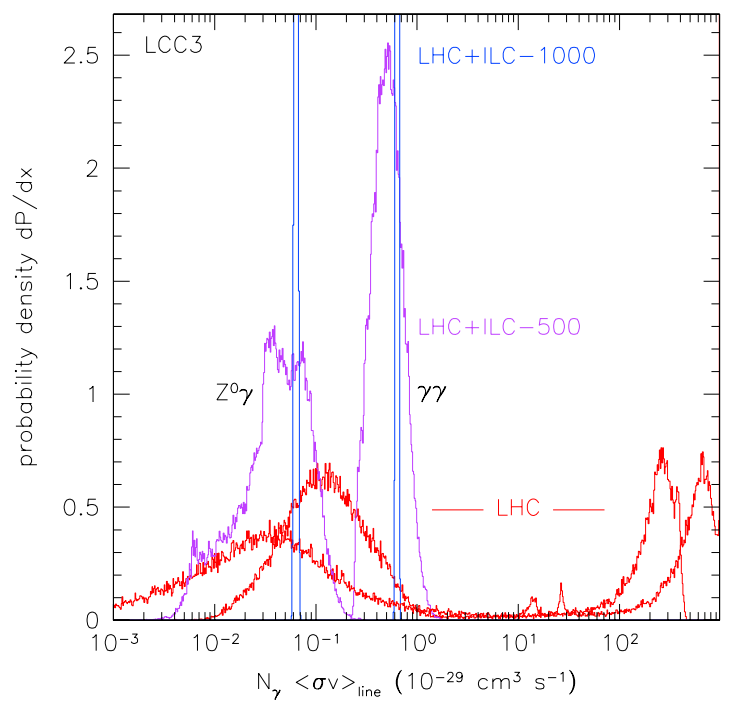

Figure 40: Gamma ray line annihilation cross section at threshold for point LCC3. The wino solution for LHC data at large cross section is clearly seen. See Fig. 8 for description of histograms.

As before, we can combine our determination of the detection cross section with the expected event yield from the SuperCDMS detector to estimate our ability to directly measure the local flux of dark matter at the earth. For this case, we expect a signal of 27 events in SuperCDMS. This gives the determination of the effective local flux of neutralinos at the Earth that is shown in Fig. 44.

\subsection{Constraints from relic density and direct detection}

Point LCC3 illustrates a scenario that in some sense is quite likely. The LHC will measure some part of the low energy spectrum of supersymmetric particles, but the mechanism for establishing relic density will be completely unknown. In this case, coannihilations with the light stau reduce the relic density by a large amount. LHC data give no hint of this as the stau is unobserved. Furthermore, only two neutralinos are observed at LHC, and no chargino is observed. Thus, the composition of the neutralinos is almost completely unknown.

Applying a relic density constraint to LHC data is a technical challenge as the range allowed is so large. Applying even the current WMAP constraint of $6 \%$ reduces the effective number of samples by a factor of 40 . Nevertheless, we can make a general statement about the composition of the lightest neutralino in that if it has too large 

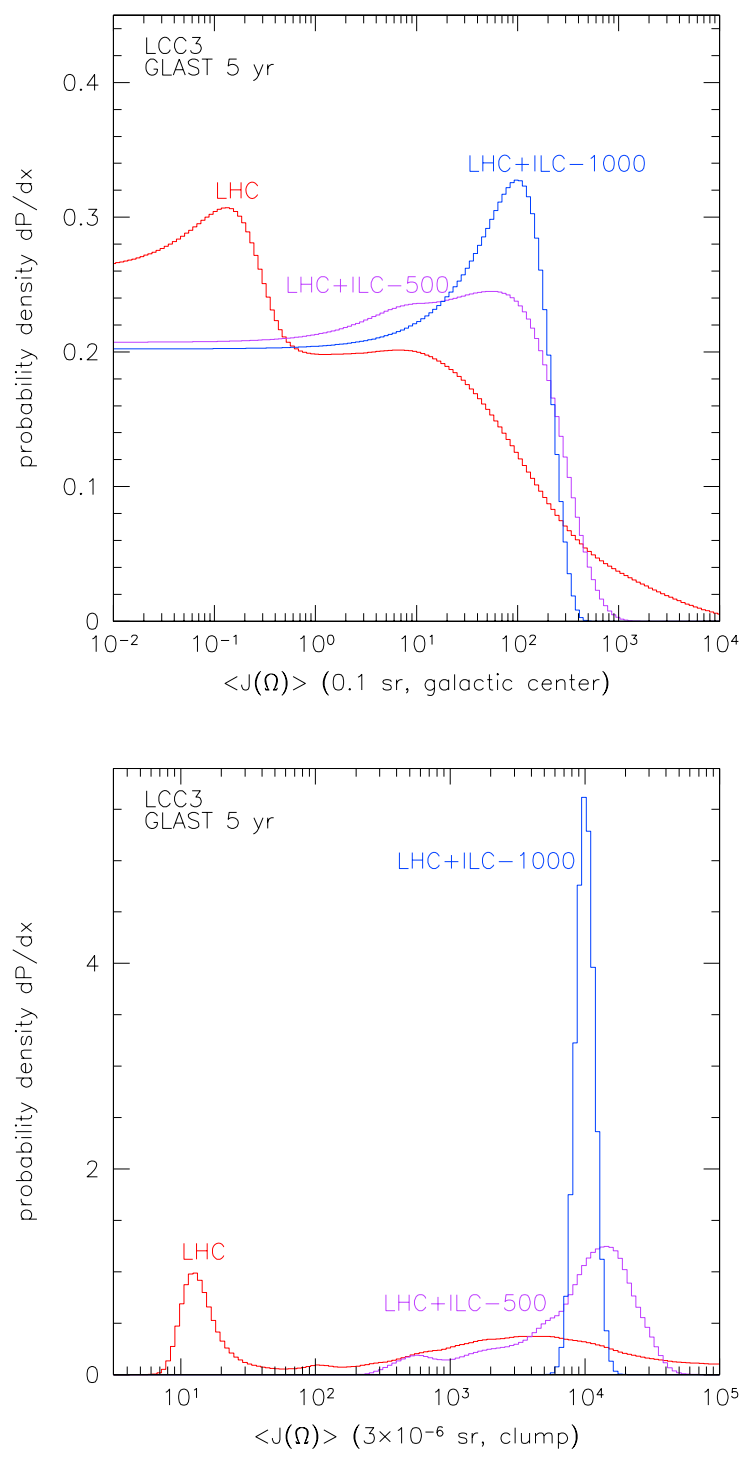

Figure 41: Halo density profiles for point LCC3: (a) galactic center, (b) dark matter clump in the galactic halo. Angle-averaged $J$ values as measured by combining a 5 -year all-sky dataset from GLAST with accelerator measurements are shown. For LHC, the wino peak at low $J$ is clearly visible. See Fig. 8 for description of histograms. 


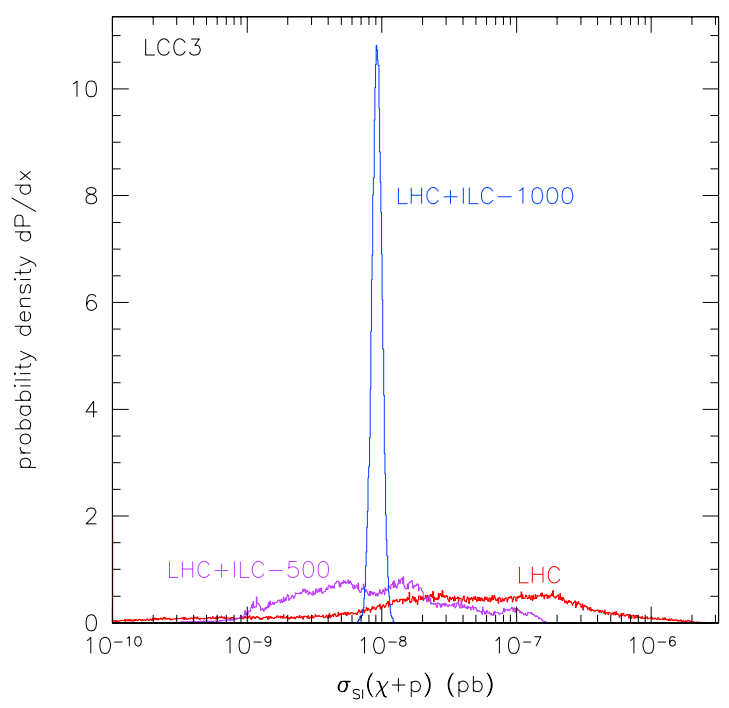

Figure 42: Spin-independent neutralino-proton direct detection cross section for point LCC3. See Fig. 8 for description of histograms.

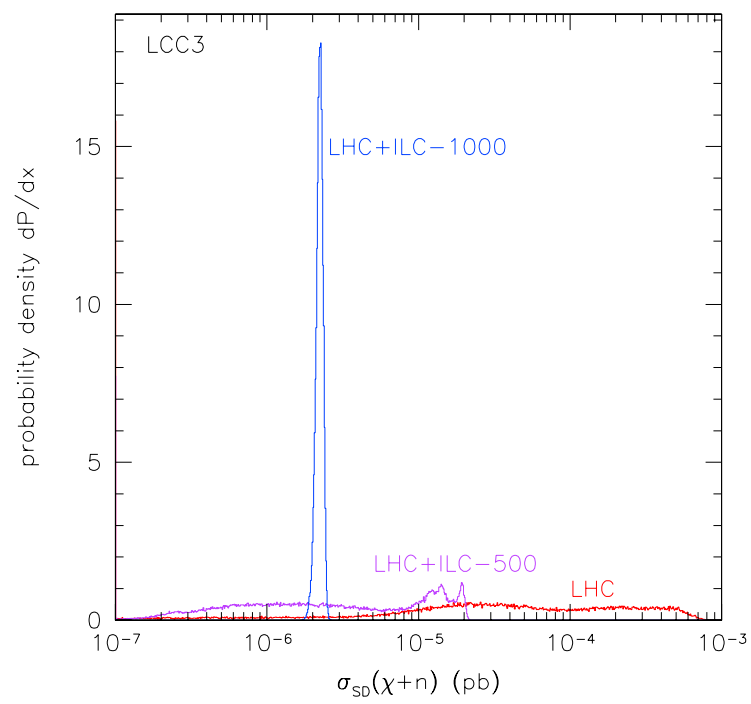

Figure 43: Spin-dependent neutralino-neutron direct detection cross section for point LCC3. See Fig. 8 for description of histograms. 


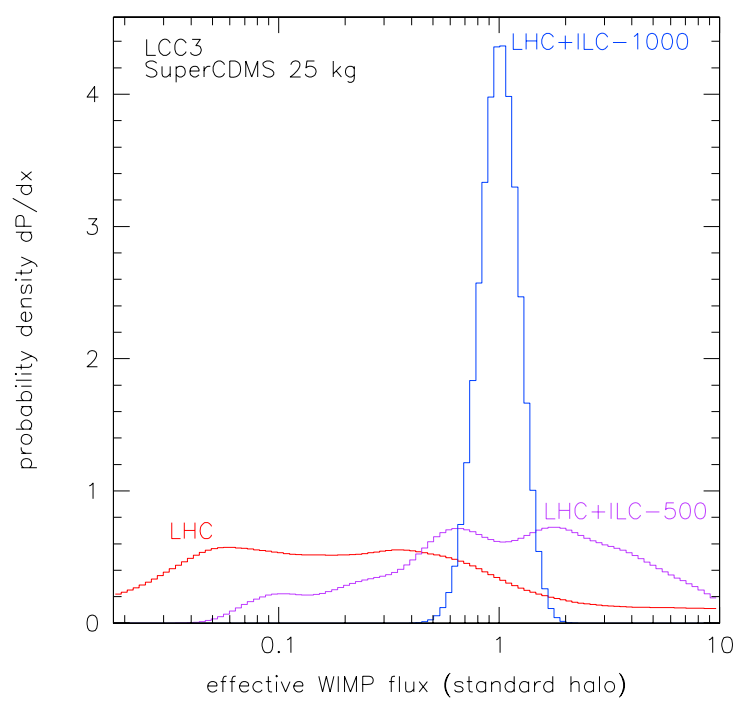

Figure 44: Effective local WIMP flux at the Earth for point LCC3. The results assume the SuperCDMS measurement described in the text. See Fig. 8 for description of histograms.

a wino or Higgsino fraction, the relic density will be too low. Thus, the relic density constraint selects the pure bino solution.

The direct detection cross section is governed by the heavy Higgs boson, seen at the LHC, and the gaugino-Higgsino mixing of the neutralino. From the cross section measured by SuperCDMS and the LHC measurement of the $H$ boson mass, it could immediately be inferred that the WIMP was a fairly pure gaugino or Higgsino $\left(Z_{g}\right.$ or $\left.Z_{h}>0.94\right)$.

The ILC-500 measurements of relic density and direct detection cross section are still quite poor. We can illustrate an interesting possibility here, that the combination of relic density and direct detection constraints can be more powerful than either alone. In particular, we study the distribution of $\tan \beta$. As shown in Fig. 45, the direct detection constraint alone has little effect. Applying the relic density constraint tightens the ILC-500 measurement considerably (and in fact reduces the ILC-1000 errors from 5\% to 3\%). Applying the direct detection constraint does have significant power if the relic density constraint is also applied, especially in the ILC-500 sample.

The ILC-1000 measures the direct detection cross section to 9\%, more accurately that it can be determined by SuperCDMS. But the relic density can only be measured to $18 \%$, so there is useful information in a cosmological relic density constraint. However, we have not been able to identify a clear beneficiary for this information. 
Somewhat surprisingly, the stau-neutralino mass splitting has only a weak correlation with relic density, given the ILC-1000 measurements (see Fig. 38).

\section{Benchmark point LCC4}

The general properties of the supersymmetry spectrum at the benchmark point LCC4 are very similar to those at LCC3. The two points differ completely, however, in the physics that establishes the neutralino relic density. At LCC3, the stau and neutralino were sufficiently close in mass that coannihilation dominated the annihilation of supersymmetric particles in the early universe. At LCC4, the parameters of the model are adjusted in a different way, such that the $A^{0}$ boson creates a resonance in neutralino annihilation near threshold.

The $A^{0}$ resonance has the potential to increase the neutralino annihilation cross section by three orders of magnitude. This implies that the influence of the $A^{0}$ typically creates a 'funnel' in the parameter space. When $m\left(A^{0}\right)=2 m(\chi)$ precisely, the annihilation cross section is very large and the neutralino relic density is essentially zero. As the $A^{0}$ moves away from the neutralino pair threshold, the annihilation cross section reverts to its typical small value. In two intermediate regions, one on each side of the threshold, the effect of the resonance is just right to produce an S-wave cross section of about $1 \mathrm{pb}$. The location of this region depends on the $A^{0}$ mass but also on the $A^{0}$ width. The width of the $A^{0}$ is sensitive to many parameters of the theory, and especially to the value of $\tan \beta$, which is difficult to determine independently. Thus, as we will see, none of the properties of the neutralino are determined particularly well at this point until the $A^{0}$ is measured in $e^{+} e^{-}$annihilation. However, once that is done, the quantities needed for the study of dark matter snap into place.

\subsection{Spectroscopy measurements}

The supersymmetry spectrum at the point LCC4 is shown in Fig. 46. The properties of the supersymmetry spectrum at LCC4 visible to the LHC and to the $500 \mathrm{GeV}$ ILC are very similar to those discussed for LCC3. The squarks and the gluino all lie below $1 \mathrm{TeV}$, so these states should be seen and characterized at the LHC. The two lightest states in the neutralino spectrum should also be visible. The parameter set of LCC4 has a large value of $\tan \beta$, making the $A^{0}$ and $H^{0}$ visible at the LHC in their $\tau^{+} \tau^{-}$decay modes. The large value of $\tan \beta$ also leads to large downward shifts of the masses of the lighter tau slepton and the bottom squark. In this case, the lighter stau is the only slepton with mass below $350 \mathrm{GeV}$, and thus it will be difficult to see any slepton at the LHC. The point LCC4 shares with LCC3 the property that only 

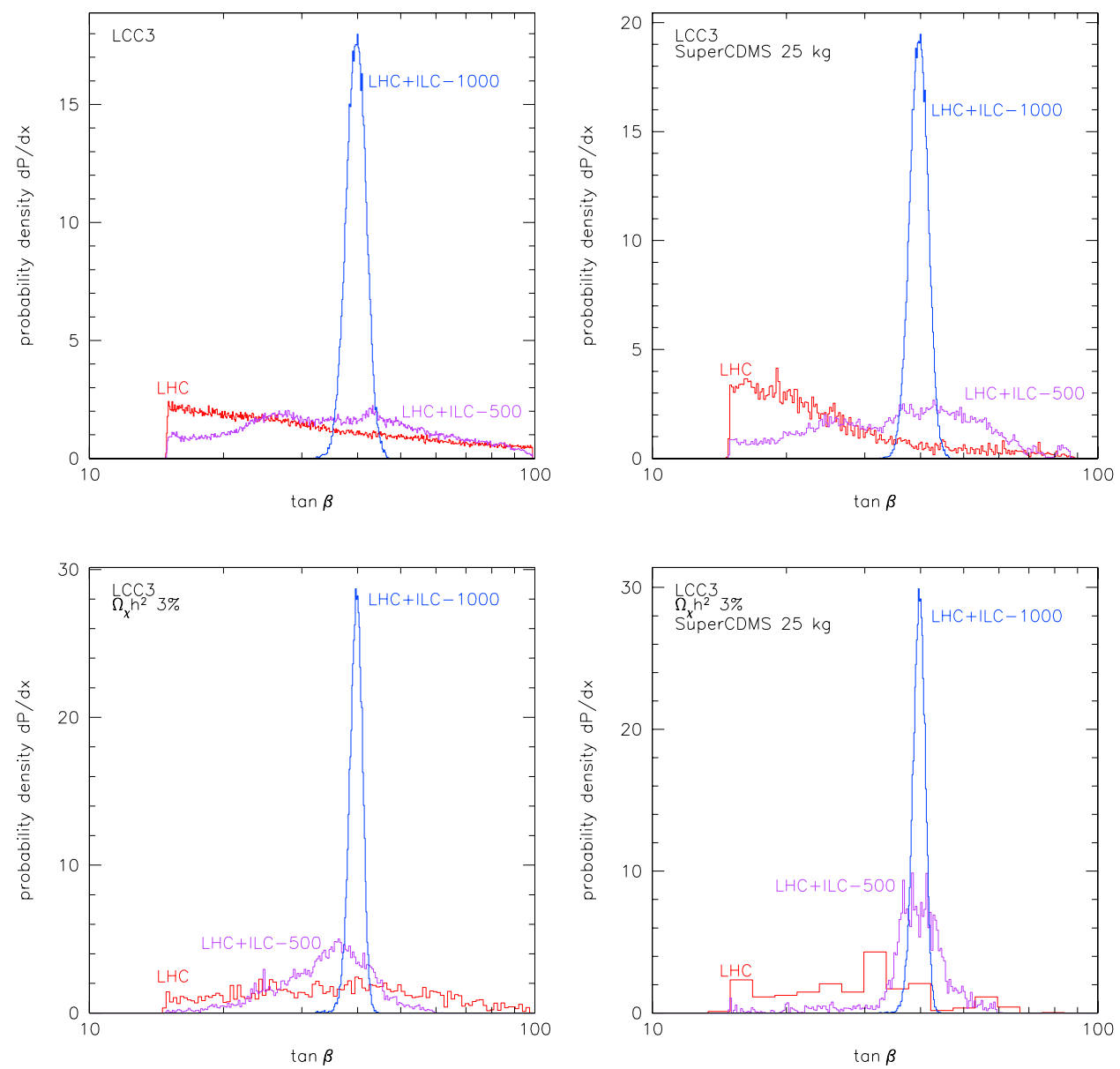

Figure 45: Measurements of $\tan \beta$ for point LCC3. Various astrophysical constraint sets are included. The relic density constraint improves even the ILC-1000 measurement, while the ILC-500 measurement is greatly helped by including both direct detection and relic density. 


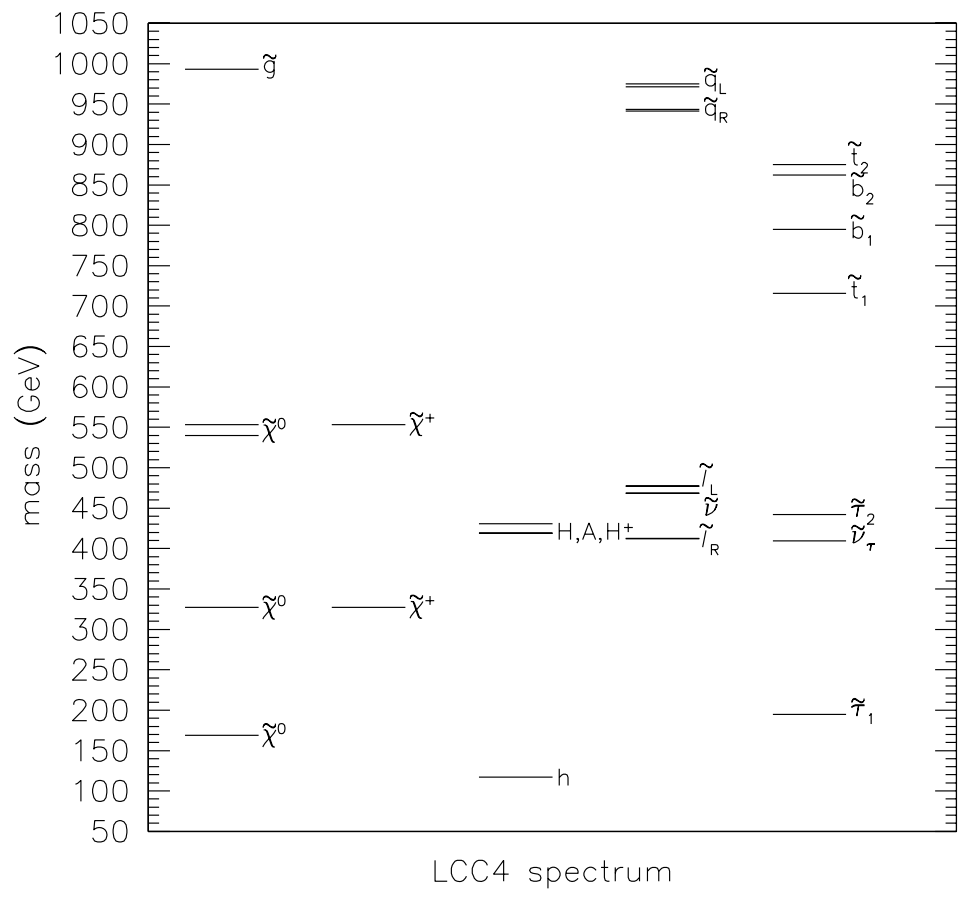

Figure 46: Particle spectrum for point LCC4. The lightest neutralino is predominantly bino, the second neutralino and light chargino are predominantly wino, and the heavy neutralinos and chargino are predominantly Higgsino. 
the few lightest supersymmetric states will be visible at the $500 \mathrm{GeV}$ stage of the ILC.

At the $1000 \mathrm{GeV}$ ILC, new features of the model can be studied in detail. The process $e^{+} e^{-} \rightarrow H^{0} A^{0}$ becomes accessible, leading to striking events with four $b$ jets. The analysis of these events has been studied in [67]. By combining the jets in pairs, it is possible to reconstruct the mass peak of the heavy Higgs bosons. The widths of the $H^{0}$ and $A^{0}$ can be measured from this mass distribution. The two peaks substantially overlap, since the widths are about $14 \mathrm{GeV}$ while the mass splitting is about $1 \mathrm{GeV}$. Since the splitting and the width are controlled by the common MSSM parameters $\tan \beta$ and $m\left(A^{0}\right)$, it is possible to fit for those parameters and improve the accuracy of the $A^{0}$ width determination.

This is the only place in the paper where we make strong use of the fact that we are restricting ourselves to the MSSM rather than working in a still more general supersymmetric model. However, in a model with a more general Higgs structure, the masses of the $H^{0}$ and $A^{0}$ would typically be distinct, and the two sets of masses and widths could be obtained separately by kinematic fitting of 4 -jet events.

The higher energy running of the ILC also makes it possible to observe the gauginos $\widetilde{\chi}_{1}^{+}$and $\widetilde{\chi}_{3}^{0}$ and to measure their masses. This gives enough information to determine the parameter $\mu$ and thus to fix the gaugino-Higgsino mixing angles.

The complete list of spectrum constraints that we expect for this point for the LHC and for each stage of the ILC is given in Tables 8 and 9.

\subsection{Relic density}

The evaluation of the relic density at LCC4 from collider data reflects the fact that the result depends sensitively on both the mass and width of the $A^{0}$ boson. At the LHC and the $500 \mathrm{GeV}$ ILC, the qualitative features of the supersymmetry spectrum are known, and the mass of the lightest neutralino and the $A^{0}$ are both known to some precision. However, this still leaves almost complete uncertainty as to the predicted value of the relic density. It is only when the width of the $A^{0}$ is measured that the actual picture begins to come into focus. This evolution is shown clearly in Fig. 47. The dependence of relic density on the $A^{0}$ width is easily seen in the ILC-500 data, illustrated in Fig. 48.

The predicted value of the relic density also depends on the gaugino-Higgsino mixing parameters. The measurement of the mass of the $\tilde{\chi}_{3}^{0}$, in particular, allows one to determine the $\mu$ parameter. This significantly improves the determination shown in Fig. 47 over scans done without this information. With the full set of collider data, we find that the relic density can be predicted to $19 \%$ accuracy. 


\begin{tabular}{lccccc} 
mass $/$ mass splitting & LCC4 value & & LHC & ILC 500 & ILC 1000 \\
\hline$m\left(\widetilde{\chi}_{1}^{0}\right)$ & 169.1 & \pm & 17.0 & - & 1.4 \\
$m\left(\widetilde{\chi}_{2}^{0}\right)$ & 327.1 & \pm & 49. & & \\
$m\left(\widetilde{\chi}_{2}^{0}\right)-m\left(\widetilde{\chi}_{1}^{0}\right)$ & 158.0 & \pm & - & - & 1.8 \\
$m\left(\widetilde{\chi}_{3}^{0}\right)-m\left(\widetilde{\chi}_{1}^{0}\right)$ & 370.6 & \pm & - & - & 2.0 \\
$m\left(\widetilde{\chi}_{1}^{+}\right)$ & 327.5 & \pm & - & - & 0.6 \\
$m\left(\widetilde{\chi}_{1}^{+}\right)-m\left(\widetilde{\chi}_{1}^{0}\right)$ & 158.4 & \pm & - & - & 2.0 \\
$m\left(\widetilde{\chi}_{2}^{+}\right)-m\left(\widetilde{\chi}_{1}^{+}\right)$ & 225.8 & \pm & - & - & 2.0 \\
\hline$m\left(\widetilde{e}_{R}\right)-m\left(\widetilde{\chi}_{1}^{0}\right)$ & 243.2 & \pm & - & - & 0.5 \\
$m\left(\widetilde{\mu}_{R}\right)-m\left(\widetilde{\chi}_{1}^{0}\right)$ & 243.0 & \pm & - & - & 0.5 \\
$m\left(\widetilde{\tau}_{1}\right)$ & 194.8 & \pm & - & 0.9 & \\
$m\left(\widetilde{\tau}_{1}\right)-m\left(\widetilde{\chi}_{1}^{0}\right)$ & 25.7 & \pm & - & 1.0 & \\
\hline$m(h)$ & 117.31 & \pm & 0.25 & 0.05 & \\
$m(A)$ & 419.3 & \pm & $1.5 *$ & - & 0.8 \\
$\Gamma(A)$ & 14.8 & \pm & - & - & 1.2 \\
\hline$m\left(\widetilde{u}_{R}\right), m\left(\widetilde{d}_{R}\right)$ & $944 ., 941$. & \pm & 94. & & \\
$m\left(\widetilde{s}_{R}\right), m\left(\widetilde{c}_{R}\right)$ & $941 ., 944$. & \pm & 97. & & \\
$m\left(\widetilde{u}_{L}\right), m\left(\widetilde{d}_{L}\right)$ & $971 ., 975$. & \pm & 141. & & \\
$m\left(\widetilde{s}_{L}\right), m\left(\widetilde{c}_{L}\right)$ & $975 ., 971$. & \pm & 146. & & \\
$m\left(\widetilde{b}_{1}\right)$ & 795. & \pm & 40. & & \\
$m\left(\widetilde{b}_{2}\right)$ & 862. & \pm & 86. & & \\
$m\left(\widetilde{t}_{1}\right)$ & 716. & \pm & $>345)$ & & \\
\hline$m(\widetilde{g})$ & 993. & \pm & 199. & & \\
& & & & &
\end{tabular}

Table 8: Superparticle masses and their estimated errors or limits for the parameter point LCC4. The notation is as in Table 6.

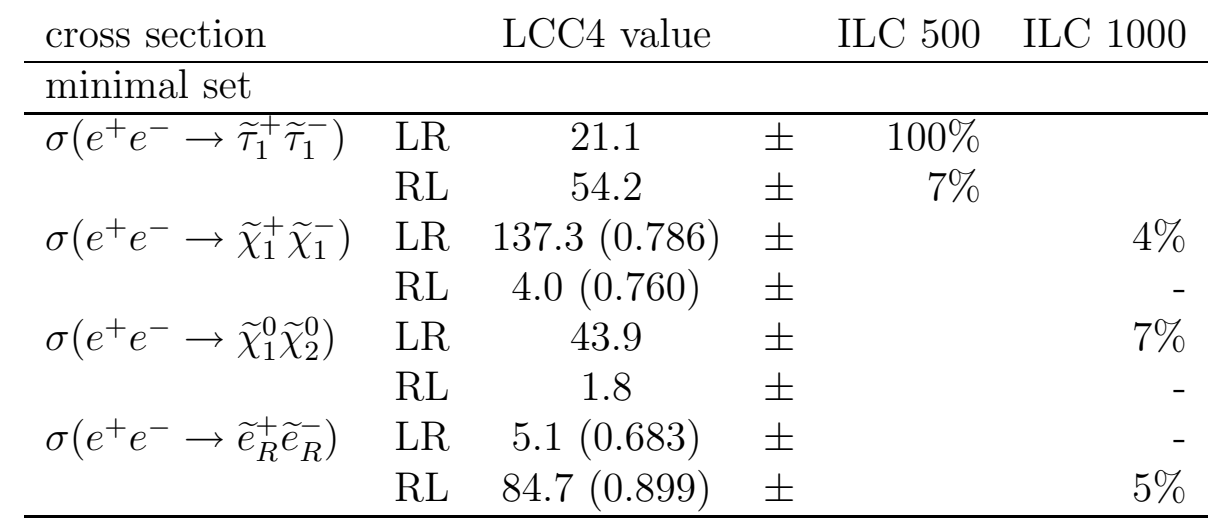

Table 9: SUSY cross sections and estimated errors for the parameter point LCC4. The notation is as in Table 3. The symbol (-) denotes that the cross section is less than $10 \mathrm{fb}$ or is otherwise not measurable. The errors are estimated using (11). 


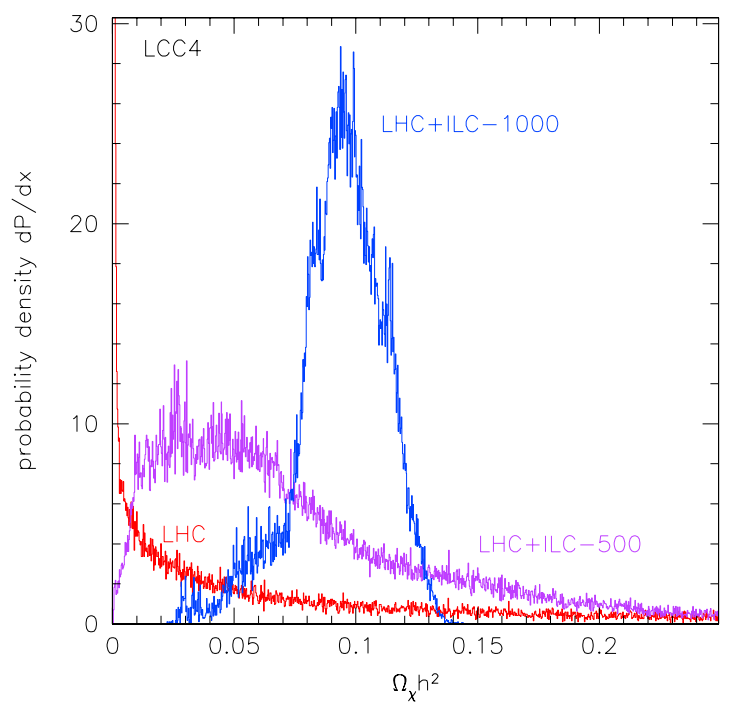

Figure 47: Relic density measurement for point LCC4. The wino peak at very small relic density is clear. See Fig. 8 for description of histograms.

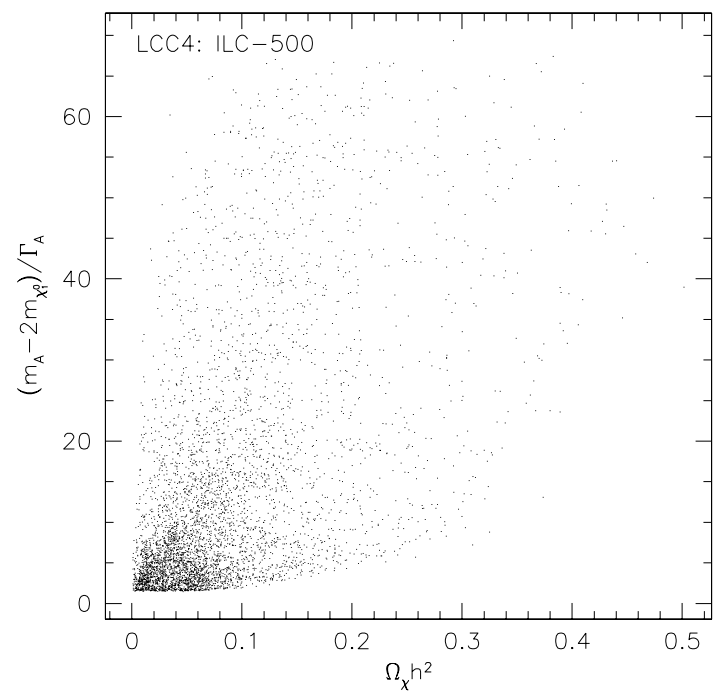

Figure 48: Scatter plot of the distance from the $A^{0}$ resonance vs. relic density for point LCC4. The quantity plotted is actually the distance in widths, with true value 5.5. 


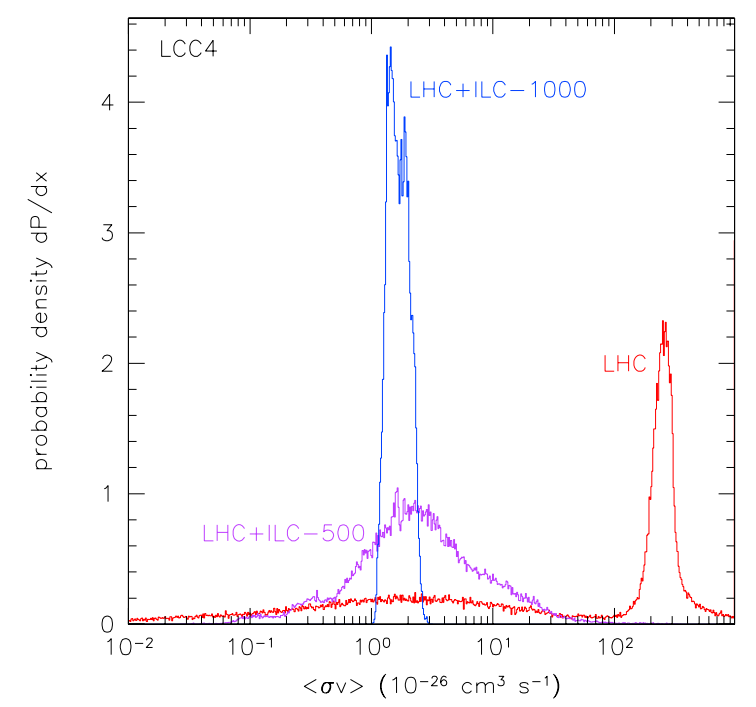

Figure 49: Annihilation cross section at threshold for point LCC4. The wino solution giving very high cross section is clearly visible. See Fig. 8 for description of histograms.

\subsection{Annihilation cross section}

In Fig. 49, we show the prediction of the neutralino annihilation cross section at threshold from collider data. Because the resonant annihilation through the $A^{0}$ is a simple S-wave process, this cross section is highly correlated with the predicted relic density discussed in the previous section. The form of the prediction is, again, complete ignorance until the $A^{0}$ width is measured, and a sharp value thereafter. The prediction of the exclusive annihilation cross sections to $\gamma \gamma$ and $\gamma Z$ follow the same pattern; this is shown in Fig. 50.

Because the annihilation cross section at threshold is large at LCC4, the collider data gives us a significant ability to interpret the counting rates from experiments that measure gamma rays from neutralino annihilation. In Fig. 51, we show the determinations of $\langle J(\Omega)\rangle$ for the galactic center and the canonical halo object described in Section 4.4. For the galactic center, we expect 8,000 signal photons, over 43,000 background; for the canonical halo object, we expect 133 signal photons, over 6 background. These signals are similar to those obtained at LCC2, and-using the very well determined annihilation cross section provided by the data from the $1000 \mathrm{GeV}$ ILC - we obtain similarly powerful results on the clustering and halo profile of dark matter. 


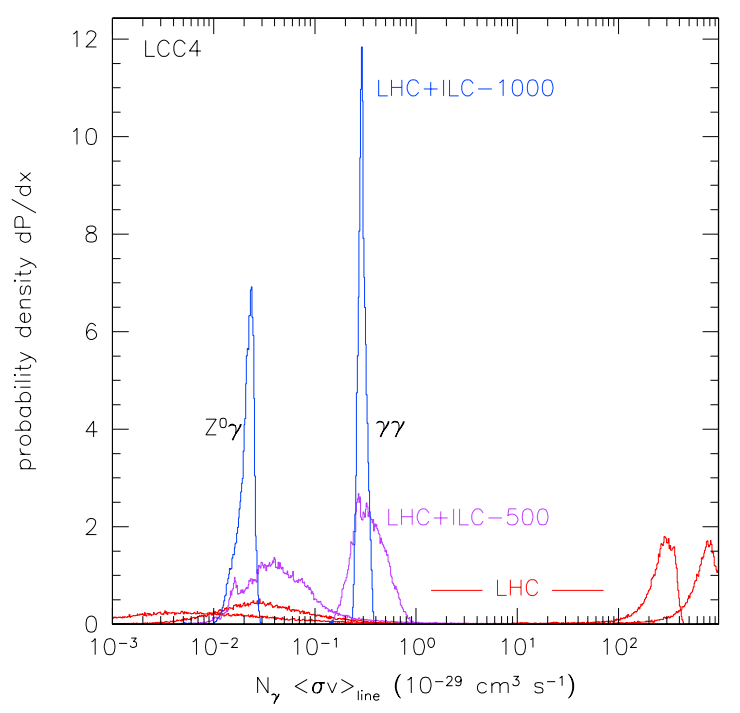

Figure 50: Gamma ray line annihilation cross section at threshold for point LCC4. Again, the wino solution at large cross section is clear. See Fig. 8 for description of histograms.

\subsection{Direct detection cross section}

Again at LCC4, the direct detection cross section is dominated by the exchange of the heavy Higgs boson $H^{0}$. The determination of this cross section from the data at the three colliders is shown in Fig. 52. The evolution is, if anything, more striking than that displayed in the previous two sections. The determination of this cross section relies crucially on the information from the $1000 \mathrm{GeV}$ ILC. The determinations of the gaugino-Higgsino mixing angles and $\tan \beta$ are crucial here as elsewhere in fixing the neutralino-Higgs couplings. The corresponding predictions for the spin-dependent part of the neutralino-neutron cross section are shown in Fig 53; these show a similar behavior.

As before, we can combine our determination of the detection cross section with the expected event yield from the SuperCDMS detector to estimate our ability to directly measure the local flux of dark matter at the earth. The direct detection cross section is the same as at LCC3, and again we expect a signal of 27 events in SuperCDMS. This gives the determination of the effective local flux of neutralinos at the Earth that is shown in Fig. 54. 

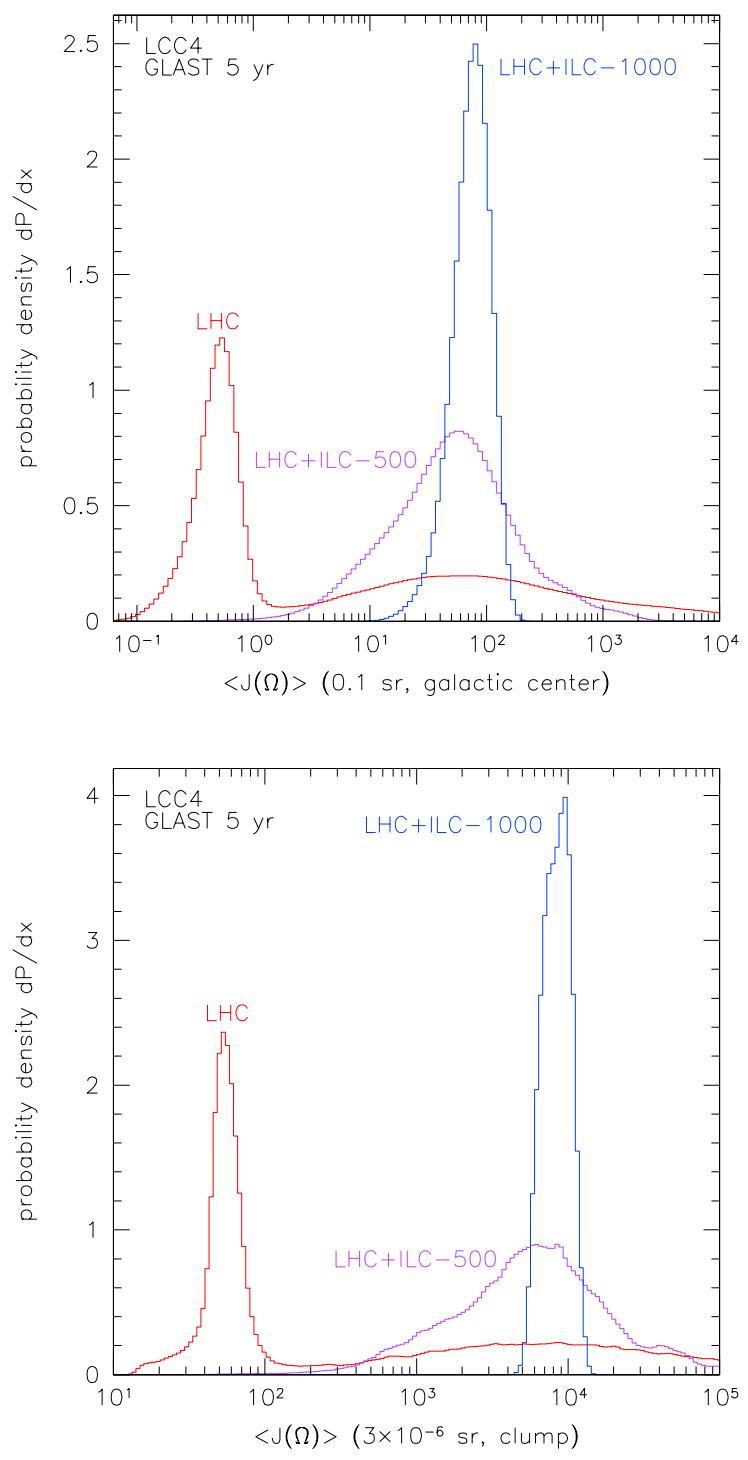

Figure 51: Halo density profiles for point LCC4: (a) galactic center, (b) dark matter clump in the galactic halo. Angle-averaged $J$ values as measured by combining a 5 -year all-sky dataset from GLAST with accelerator measurements are shown. See Fig. 8 for description of histograms. 


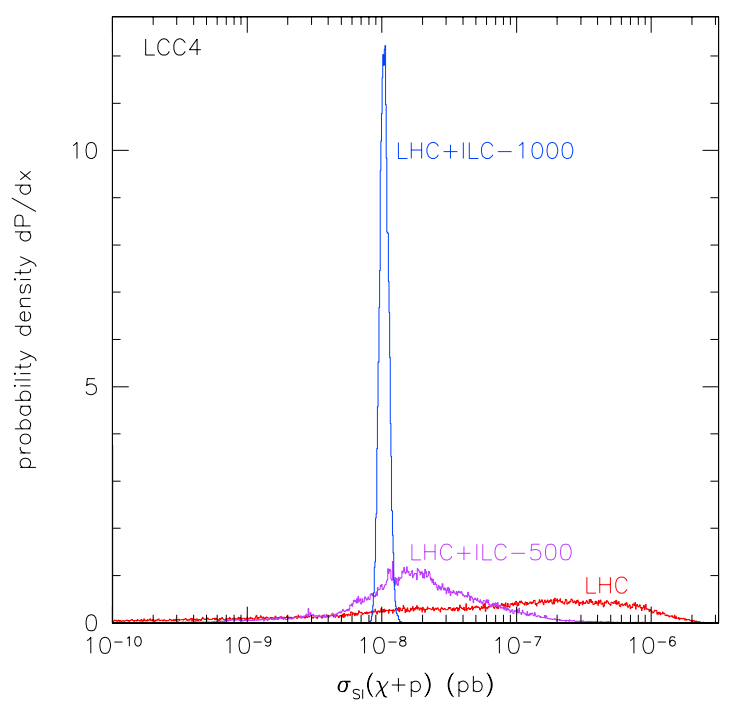

Figure 52: Spin-independent neutralino-proton direct detection cross section for point LCC4. See Fig. 8 for description of histograms.

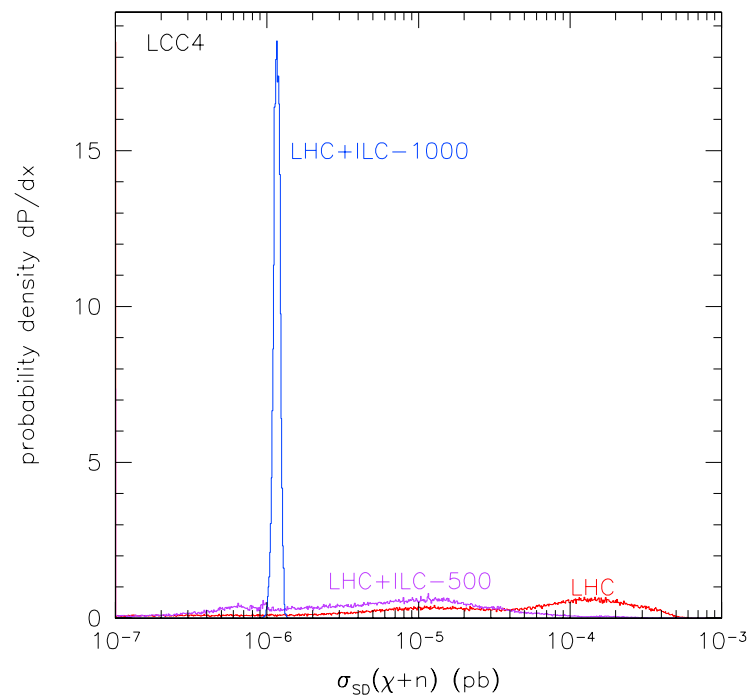

Figure 53: Spin-dependent neutralino-neutron direct detection cross section for point LCC4. See Fig. 8 for description of histograms. 


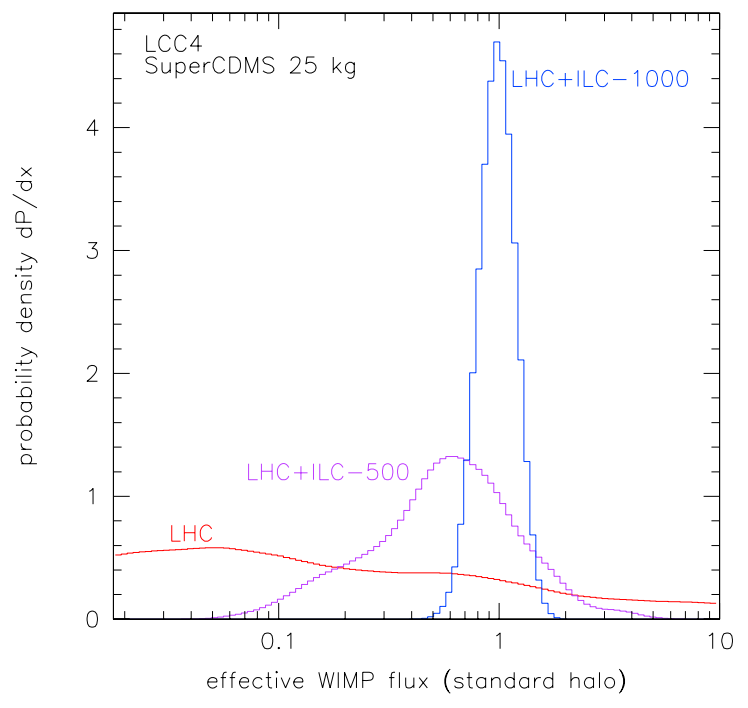

Figure 54: Effective local WIMP flux at the Earth for point LCC4. The results assume the SuperCDMS measurement described in the text. See Fig. 8 for description of histograms.

\subsection{Constraints from relic density and direct detection}

Point LCC4 is quite similar to point LCC3, so much of the discussion of section 6.5 relevant to the LHC applies. In particular, any relic density constraint applies fixes the neutralino to be almost pure bino. However, like LCC3, there is little hint in LHC data of the mechanism for establishing the correct relic density, in this case resonant annihilation through the CP-odd Higgs $A$.

The ILC-1000 will measure the direct detection cross section to $7.5 \%$ accuracy, essentially perfect accuracy given astrophysical uncertainties. It measures the relic density at the $19 \%$ level. But, as we found at LCC3, there is no clear fundamental parameter estimate that would benefit greatly from a cosmological constraint. There is a mild correlation between relic density and the parameter $\left(m_{A}-2 m_{\chi_{1}^{0}}\right) / \Gamma_{A}$ (distance in widths from the $A$ resonance), but it is not spectacular. One thing that is greatly helped is the annihilation cross section at $v=0$. Since the annihilation is resonant, the cross section at freeze-out velocities is not much different, though the correlation is not as tight as with LCC2. Applying a $1 \%$ relic density constraint changes the accuracy of the annihilation cross section estimate from $20 \%$ to $5 \%$, a quite significant improvement.

Astrophysical constraints can provide a significant information on the annihilation cross section. Direct detection can reduce the significance of the wino peak, while a 
relic density constraint completely removes it. We illustrate this point in Fig. 55.

\section{Neutralino annihilation products}

In this section, we consider in more detail the computation of indirect signals of dark matter annihilation from observations of gamma rays and positrons that are produced in neutralino pair annihilation. We leave discussion of neutrinos, antiprotons, and antideuterons to future work.

In the previous few sections, we presented estimates of the neutralino annihilation cross section that might be obtained from collider data on the SUSY spectrum. However, as we have remarked in Section 2.7, there is a simpler way to obtain what would seem to be an acceptably accurate prediction. We start from a value of the neutralino mass obtained from LHC data. If we assume that the neutralino makes up the bulk of the dark matter, we can use the cross section (2) derived from the relic density as an estimate of the astrophysical annihilation cross section. We assume that the neutralinos annihilate to hadronic jets, either in direct decays to quarks or through decays to $W$ and $Z$ bosons. This gives a roughly universal spectrum of energies for the annihilation products, scaling with the neutralino mass. The arguments are robust and simple to implement. But are they correct?

\subsection{Gamma ray spectra}

The argument we have just given works best for the gamma ray spectra from dark matter annihilation. In Fig. 56, we show the gamma ray spectra computed from the model parameters at the four LCC points. The four spectra are compared to reference spectra with the shape of the LCC4 spectrum, scaled horizontally to the correct endpoint at $E_{\gamma}=m\left(\chi_{1}^{0}\right)$, and normalized so that the peak cross section is proportional to the total annihilation cross section. We have taken the reference shape from LCC4 because, in this case, the dominant annihilation reaction goes to the 2-jet final state $b \bar{b}$. We see that the approximation is an excellent one for all four models.

It is not surprising that the shapes of the spectra are similar for the cases of LCC1, LCC3, and LCC4. In all four cases, the annihilation at threshold is dominated by the process $\chi \chi \rightarrow b \bar{b}$, with subsequent evolution of the jets into $\pi^{0}$ 's and the decay of these to gammas. However, it is quite surprising that the spectrum for LCC2, which is dominated by the processes $\chi \chi \rightarrow W^{+} W^{-}, Z^{0} Z^{0}$, yields the same spectrum. Apparently, the gamma ray spectra from hadronic jets, whatever their origin, do have the universal form assumed in our argument above. 

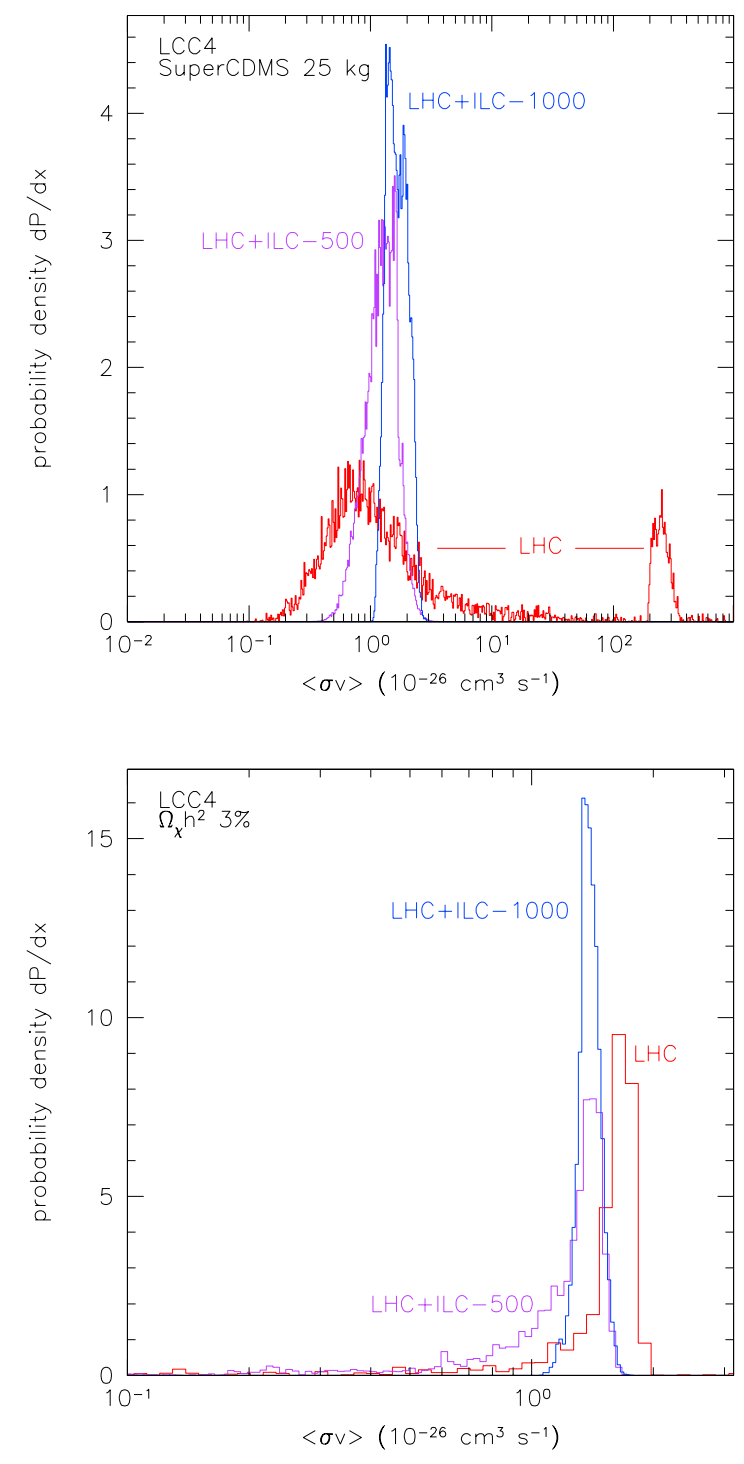

Figure 55: Annihilation cross section at threshold for point LCC4, including various astrophysical constraints. Direct detection reduces the weight of the wino solution, and relic density completely eliminates this possibility. 

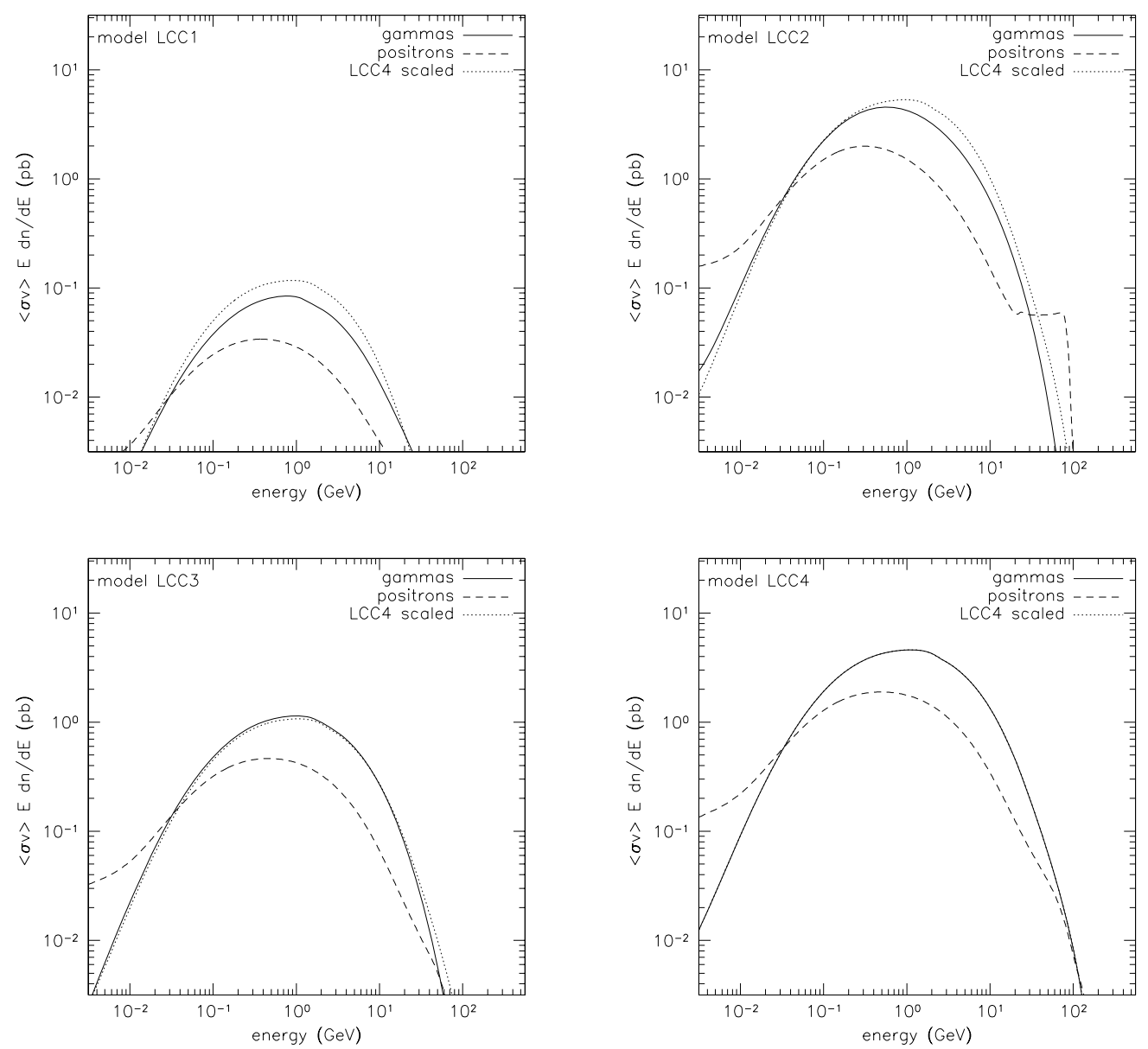

Figure 56: Spectra of gamma rays and positrons from neutralino annihilation at threshold for points LCC1-4. Solid curves are gammas, dashed curves are positrons, and dotted curves show the gamma spectrum of LCC4, scaled to each endpoint and total cross section. The scaled LCC4 spectrum is a good match in every case. The positron spectrum for LCC2 exhibits a shelf due to direct decays of gauge bosons, e.g. $\chi \chi \rightarrow W^{+} W^{-} \rightarrow e^{+} \nu \bar{u} d$. 


\begin{tabular}{lllllll} 
Process & & & & LHC & ILC-500 & ILC-1000 \\
\hline LCC1: & & & & & & \\
$\chi \chi \rightarrow$ & $b \bar{b}$ & 0.629 & \pm & $46 \%$ & $46 \%$ & $15 \%$ \\
& $\tau^{+} \tau^{-}$ & 0.282 & \pm & $79 \%$ & $71 \%$ & $43 \%$ \\
& $W^{+} W^{-}$ & 0.046 & \pm & $65 \%$ & $40 \%$ & $13 \%$ \\
& $\gamma \gamma$ & 0.016 & \pm & $54 \%$ & $42 \%$ & $10 \%$ \\
& $g g$ & 0.013 & \pm & $52 \%$ & $41 \%$ & $11 \%$ \\
LCC2: & & & & & & \\
$\chi \chi \rightarrow$ & $W^{+} W^{-}$ & 0.868 & \pm & $11 \%$ & $11 \%$ & $1.4 \%$ \\
& $Z^{0} Z^{0}$ & 0.114 & \pm & $66 \%$ & $12 \%$ & $3.7 \%$ \\
LCC3: & & & & & & \\
$\chi \chi \rightarrow$ & $b \bar{b}$ & 0.974 & \pm & $70 \%$ & $36 \%$ & $1.3 \%$ \\
& $\tau^{+} \tau^{-}$ & 0.014 & \pm & $113 \%$ & $93 \%$ & $92 \%$ \\
LCC4: & & & & & & \\
$\chi \chi \rightarrow$ & $b \bar{b}$ & 0.889 & \pm & $70 \%$ & $16 \%$ & $16 \%$ \\
& $\tau^{+} \tau^{-}$ & 0.103 & \pm & $90 \%$ & $50 \%$ & $52 \%$
\end{tabular}

Table 10: Branching ratios in neutralino pair annihilation. The last three columns give the fractional error (variance/mean) from the MCMC scans.

In addition, the discrepancy in the normalizations is readily understood. The relic density of WIMP dark matter is established at a small but nonzero temperature, $T / m \sim 1 / 25$. The cross section relevant to gamma ray detection of WIMP is that almost precisely at threshold. In cases in which the dominant modes of annihilation proceed in the S-wave, (2) is a reasonable approximation to the correct cross section. We find for the neutralino annihilation cross section at threshold $\sigma v=0.55 \mathrm{pb}$ for LCC2 and $\sigma v=0.48 \mathrm{pb}$ for LCC4. However, at LCC1, the dominant modes of annihilation for the purpose of computing the relic density are $\mathrm{P}$-wave annihilations to $\ell^{+} \ell^{-}$. These cross sections are very small at threshold; the dominant process just at threshold is the subdominant reaction $\chi \chi \rightarrow b \bar{b}$. At the point LCC3, the relic density is set by coannihilation processes such as $\widetilde{\tau}^{-} \widetilde{\tau}^{-} \rightarrow \tau^{-} \tau^{-}$. In present astrophysical conditions, all of the $\widetilde{\tau}$ 's have decayed away. At both LCC1 and LCC3, $b \bar{b}$ appears because this is the fermion-antifermion final state with the least amount of helicity suppression. We find, for the neutralino annihilation cross section at threshold for these points, $\sigma v=0.012 \mathrm{pb}$ for LCC1 and $\sigma v=0.11 \mathrm{pb}$ for LCC3.

If more incisive probes of WIMP annihilation are available, it might be important to know what are the fractions of the total annihilation rate that go to the various possible final states. In Table 10, we present the branching fractions to the most important final states for the four reference points, and the estimates of these branching fractions that we obtain from the three sets of collider constraints. 


\subsection{Positron spectra}

We have just shown that the gamma ray spectrum resulting from neutralino annihilation is remarkably independent of the model. It is controlled almost entirely by the total neutralino pair annihilation cross section, and, through this, is often determined if the physics of neutralino annihilation is understood even qualitatively. For other annihilation products, however, the story can be quite different. In this section, we will discuss the visibility of the positron signal of dark matter annihilation in our four models. Similar considerations apply to neutrino signals. However, since in all four of our models the neutralino mass is below $200 \mathrm{GeV}$, neutrinos from neutralino annihilation will be produced at too low an energy to be visible above the thresholds of cosmic-ray neutrino detectors such as ICE-CUBE.

Unlike gamma rays, which fly directly from the source to a detector on earth, positrons execute a random walk in the galactic magnetic field, losing energy continually along the way. Thus, positrons from WIMP annihilation that are observed at the earth must originate inside the galaxy, within a few kpc. The annihilation rate depends on $\rho^{2}(x)$ averaged over this volume, a quantity closely related to the local halo density discussed in relation to direct detection, but possibly enhanced by local clumpiness parametrized by the boost factor (8). The energy loss in propagation favors the highest-energy positrons in the spectrum.

The propagation of positrons through the galaxy has been modeled quantitatively in $[92,93]$. We have found it an interesting exercise to fold the positron spectra for our four models with the smearing predicted by the model of [93]. The results are shown in Fig. 57.

The signal from LCC2 is larger than the others, for several reasons. First of all, this point has a full strength total annihilation cross section. In addition, as one can see from Fig. 56, the positron spectrum at this point has a component with a flat distribution extending to the kinematic endpoint. This results from the fact that the dominant annihilation reactions at LCC2 are to $W^{+} W^{-}$and $Z^{0} Z^{0}$, that is, to vector bosons that have direct two-body decays to positrons. The sharp feature at the extreme right of Fig. 56(b) is transformed into the peak at the high-energy edge of the LCC2 curve in Fig. 57.

We can compare this spectrum to the recent measurement of the cosmic-ray positron spectrum by the HEAT experiment. The comparison is shown in Fig. 58 . The HEAT data shows an anomaly at its upper edge, with a cross section that is roughly flat in a region in which the background is expected to be decreasing. As the figure shows, we can fit this with the positron spectrum from LCC2. To obtain the correct normalization, we must enhance the production over that for a smooth distribution of dark matter with density $\rho_{0}=0.3 \mathrm{GeV} \mathrm{cm}^{-3}$ by assuming a large 


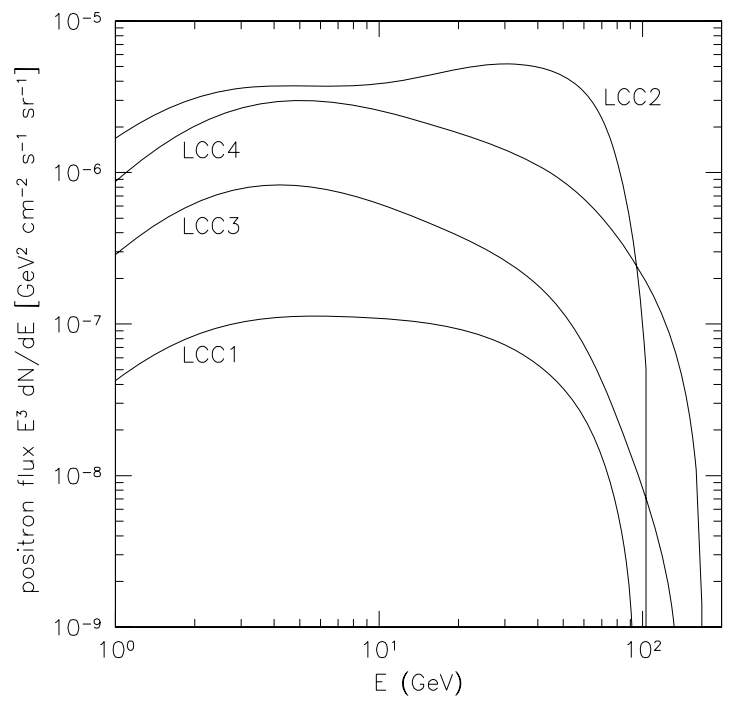

Figure 57: Spectrum of positrons for all models after galactic propagation effects are accounted for. The "interstellar" spectrum is illustrated. Solar modulation is neglected. Above a few $\mathrm{GeV}$ solar modulation effects are negligible.

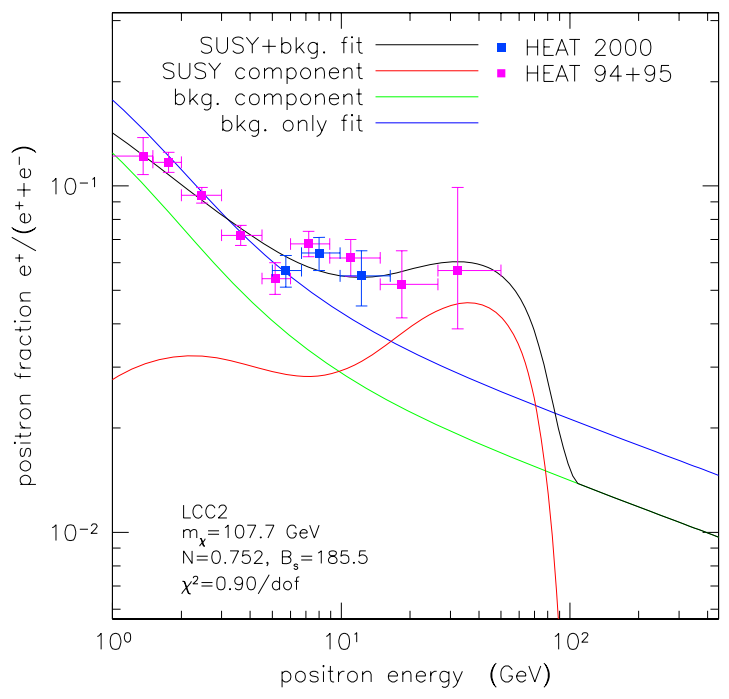

Figure 58: Positrons observed on earth, as a fraction of electrons, for LCC2. The HEAT data are plotted as well, indicating the possibility of an excess. The positron signal from a smooth halo has been boosted by a factor of 185 in order to fit the data. 
boost factor $B=190$.

Is this a correct way to interpret the data? We ourselves are skeptical. Nevertheless, if we knew from collider measurements that the WIMP mass was $100 \mathrm{GeV}$ and that we were in a region of the parameter space that favored annihilation through $\chi \chi \rightarrow W^{+} W^{-}$, this fit to the data might be considered compelling evidence for neutralino annihilation and for a significantly clumpy distribution of WIMPs in the galaxy.

\section{Recap: Collider determination of WIMP properties}

Our analysis in this paper has presented a series of worked examples that illustrate the interactions we might expect over the coming years between astrophysical dark matter detection experiments and high-energy physics experiments that will measure the spectrum of new particles in the hundred-GeV mass range. In this section, we will assemble the results that we have obtained and give our interpretation of their implications.

\subsection{Summary of results: cross sections}

In Table 11, we summarize the results of our Monte Carlo scans for the most important WIMP parameters that are determined by collider measurements of supersymmetry spectroscopy: the relic density $\Omega_{\chi} h^{2}$, the annihilation cross section at threshold $\sigma v$ (in $\mathrm{pb}$ ), and the spin-independent scattering cross section on protons $\sigma(\chi p)$ (in units of $10^{-8} \mathrm{pb}$ ). The results are given for each of the four benchmark models and for each of the three sets of collider measurements discussed in Sections 4-7. The results are quoted as a percentage error, defined as the variance divided by the mean, computed from the statistical sample generated by our Monte Carlo process. A large variance indicates a poor determination of the model parameters and might also indicate the presence of multiple solutions. For the correct interpretation, one should look at the detailed shapes of the probability distributions shown in the figures in Sections 4-7. For reference, the mean values of the Monte Carlo samples

are listed in the last three columns of the table when these differ by $10 \%$ or more from the nominal value.

We emphasize that all of these cross section determinations are 'model-independent' in the following sense: We assume that it has been shown from collider data that the model of new physics at the hundred GeV scale is supersymmetry. We then fit the collider data on new particles to the Minimal Supersymmetric Standard Model, studying the model in complete generality with 24 free parameters. We have shown in 


\begin{tabular}{|c|c|c|c|c|c|c|c|}
\hline & $\Omega h^{2}$ & LHC & ILC-500 & ILC-1000 & $\begin{array}{r}\mathrm{LHC} \\
(\text { mean })\end{array}$ & ILC-500 & ILC-1000 \\
\hline LCC1 & 0.192 & $7.2 \%$ & $1.8 \%$ & $0.24 \%$ & & & \\
\hline LCC2 & 0.109 & $82 . \%$ & $14 . \%$ & $7.6 \%$ & 0.074 & & \\
\hline LCC3 & 0.101 & $167 \%$ & $50 . \%$ & $18 . \%$ & 0.24 & & \\
\hline \multirow[t]{2}{*}{$\mathrm{LCC} 4$} & 0.114 & $405 \%$ & $85 . \%$ & $19 . \%$ & 0.26 & 0.083 & 0.094 \\
\hline & $\sigma v$ & & & & (mean) & & \\
\hline LCC1 & 0.0121 & $165 . \%$ & $54 . \%$ & $11 . \%$ & 0.0069 & & \\
\hline LCC2 & 0.547 & $143 . \%$ & $32 . \%$ & $8.7 \%$ & 8.47 & & \\
\hline LCC3 & 0.109 & $154 . \%$ & $178 . \%$ & $10 . \%$ & 24.2 & 0.311 & \\
\hline \multirow[t]{2}{*}{ LCC4 } & 0.475 & $557 . \%$ & $228 . \%$ & $20 . \%$ & 82.5 & 1.83 & 0.57 \\
\hline & $\sigma(\chi p)$ & & & & (mean) & & \\
\hline LCC1 & 0.418 & $44 . \%$ & $45 . \%$ & $5.7 \%$ & 0.20 & & \\
\hline LCC2 & 1.866 & $62 . \%$ & $63 . \%$ & $22 . \%$ & 3.57 & 2.82 & 2.19 \\
\hline LCC3 & 0.925 & $184 . \%$ & $146 . \%$ & $8.6 \%$ & 13.2 & 1.86 & \\
\hline LCC4 & 1.046 & $150 . \%$ & $190 . \%$ & $7.5 \%$ & 23.2 & 3.59 & \\
\hline
\end{tabular}

Table 11: Fractional errors in the determination of the most important microscopic WIMP parameters derived from the MCMC scans: $\Omega h^{2}$, the predicted relic density, $\sigma v$, the annihilation cross section at threshold (in pb), and $\sigma(\chi p)$, the spin-independent neutralino-proton cross section (in units of $10^{-8} \mathrm{pb}$ ). The second column lists the values predicted by the benchmark models. Columns $3-5$ give the fractional error (variance/mean) from the MCMC scans. Columns 6-8 give the mean value found from the MCMC data when this deviated by more than $10 \%$ from the nominal value in column 2. As discussed in Appendix A, the quoted errors are accurate to $10 \%$ or better, e.g. a $20 \%$ error is $20 \% \pm 2 \%$.

many examples given in Section 4-7 that this parametrization is sufficiently general to allow all of the possible physical mechanisms that are expected in this general class of models to be considered in the fitting procedure. Thus we believe that we have justified the claim made in Section 3.2 that cross section estimates obtained from collider data through an analysis such as ours can be used without undue qualification to analyze astrophysical measurements.

The table shows that, for all of the quantities listed, carrying out the full program of collider measurements that we have presented results in a clear determination of the microscopic cross sections that can be used to interpret astrophysical dark matter observations. The precise quality of the determination depends on the specific scenario. In scenarios in which the dominant annihilation mechanism is through simple annihilation to leptons, we find that the accuracy of the prediction of the relic density is at the level of a fraction of a percent, comparable to the best measurements of the 
dark matter density from the cosmic microwave background expected in that era. In scenarios in which special relations among the superparticle masses are necessary for rapid enough annihilation, the quality of the microscopic prediction decreases and we find an accuracy of about $20 \%$. In all cases, the agreement of the microscopic and astrophysical determinations within the errors would be nontrivial and would provide striking evidence that the particle identified at colliders is indeed the dominant component of astrophysical dark matter.

We believe that the accuracies we have listed are the best ones that can be obtained at the current level of our understanding of the experimental capabilities of the next-generation colliders. That is, we could not find additional measurements beyond the ones we have listed that would significantly improve the accuracies we have quoted for the neutralino cross sections. We note, though, that, in the linear collider studies, relatively little effort has gone into analyses aimed at very accurate cross section measurements. We have seen that the ILC cross sections can be very important in fixing the gaugino-Higgsino mixing angles and $\tan \beta$, parameters that provide a major uncertainty in the neutralino properties. If cross sections could be measured at the ILC with errors considerably smaller than the estimate (11), the output accuracies would improve. To do this, however, it is necessary to confront the Standard Model backgrounds to the observations of supersymmetric particles and to model and subtract these backgrounds with very high precision.

We have also shown that the collider data predicts the WIMP annihilation cross section and the cross section relevant to direct detection at the $20 \%$ level or better in all of the cases that we have considered. This is quite sufficient to use the microscopically-determined cross sections to provide strong constraints on the distribution of the observed WIMPs in the galaxy.

\subsection{Summary of result: astrophysics}

In Table 12 we summarize the constraints for the three specific model problems that we have considered in this paper, the determination by GLAST of the dark matter density integral $J \sim \int d z \rho^{2}$ near the galactic center, the determination by GLAST of the value of $J$ for a representative clump of dark matter in the halo of the galaxy, and the determination by SuperCDMS of the local flux of dark matter at a location on Earth. The specific parameters assumed for the detectors and sources were detailed in Section 4.4 and 4.5. We emphasize that we have chosen these specific experiments as representative examples meant to illustrate the implications of the collider measurements for the many dark matter observation experiments that will be carried out over the next decade.

The results given in the table for determinations of the density integral $\langle J\rangle$ reflect 


\begin{tabular}{|c|c|c|c|c|c|c|c|}
\hline & $\langle J\rangle(\mathrm{gc})$ & $\mathrm{LHC}$ & ILC-500 & ILC-1000 & $\begin{array}{r}\text { LHC } \\
\text { limits } \\
\end{array}$ & $\begin{array}{r}\text { ILC-500 } \\
(95 \% \text { CL }) \\
\end{array}$ & ILC-1000 \\
\hline LCC1 & 100 & & & & $<3100$ & $<1400$ & $<1200$ \\
\hline LCC2 & 100 & $174 . \%$ * & $32 . \%$ & $29 . \%$ & & & \\
\hline LCC3 & 100 & & & & $<268$ & $<203$ & $<154$ \\
\hline \multirow[t]{2}{*}{$\mathrm{LCC} 4$} & 100 & $242 . \%$ * & $202 . \%$ & $36 . \%$ & & & \\
\hline & $\langle J\rangle($ clump $)$ & & & & limits & $(95 \%$ CL) & \\
\hline LCC1 & 10,000 & & & & $<59,000$ & $<29,000$ & $<23,000$ \\
\hline LCC2 & 10,000 & $64 . \% *$ & $18 . \%$ & $12 . \%$ & & & \\
\hline LCC3 & 10,000 & $166 . \%$ * & $77 . \%$ & $21 . \%$ & & & \\
\hline \multirow[t]{2}{*}{$\mathrm{LCC} 4$} & 10,000 & $139 . \%$ * & $133 . \%$ & $21 . \%$ & & & \\
\hline & $\Phi_{\text {local }} / \Phi_{0}$ & & & & limits & $(95 \% \mathrm{CL})$ & \\
\hline$\overline{\mathrm{LCC} 1}$ & 1. & & $55 . \%$ & $28 . \%$ & $>0.95$ & & \\
\hline $\mathrm{LCC} 2$ & 1. & $28 . \%$ * & $36 . \%$ & $22 . \%$ & & & \\
\hline LCC3 & 1. & & $140 . \%$ & $21 . \%$ & $>0.016$ & & \\
\hline LCC4 & 1. & & $249 . \%$ & $19 . \%$ & $>0.010$ & & \\
\hline
\end{tabular}

Table 12: Errors or limits for the illustrative astrophysical measurements that we have presented in Section 4-7. The quantities considered are: $\langle J\rangle_{g c}$, the average of the density integral $J \sim \int d z \rho^{2}$ near the galactic center, $\langle J\rangle_{\text {clump }}$, the average of $J$ in a small circle around the center of a typical clump of dark matter in the galactic halo, and $\Phi_{l o c a l}$, the effective flux of dark matter impinging on a direct detection experiment on earth, normalized to a standard halo distribution. The output values quoted include the expected experimental errors. More details of the assumptions involved in these analyses are given in Sections 4.4 and 4.5. The second column lists the assumed astrophysical values. Columns $3-5$ give the fractional error (variance/mean) from the MCMC scans. Columns 6-8 give 95\% upper or lower confidence limits based on the MCMC data, as appropriate. For each situation, either an error or a limit is quoted. In the cases labeled by ${ }^{*}$, the MCMC data includes multiple solutions, and we restrict our calculation to points in the neighborhood of the correct solution. See the text for further discussion of all of these points. As discussed in Appendix A, the quoted errors for the microscopic quantities (annihilation cross section, direct detection cross section) are accurate to $10 \%$ or better. 
several factors in addition to the accuracies given in Table 11 with which the microscopic WIMP cross section will be known. They include also the statistics of the observation, and the error from the expected uncertainty in the background. These two factors depend in turn on the underlying physics scenario and on the value of the relevant WIMP cross section. If the cross section is small, the statistical error will be relatively large, and the background will be more important relative to the signal.

These contributions are reflected in our results on $\langle J\rangle$ from the galactic center in the following way. The points LCC1 and LCC3 have suppressed annihilation cross sections at threshold. In these cases, the background always dominates, and we can only set upper limits on the density integral. However, the lower bounds on the cross sections that we obtain from the collider data allow these upper limits to significantly exclude dark matter distributions that are highly peaked at the galactic center. For LCC2 and LCC4, the annihilation cross section is at the full strength expected from (2), and a robust signal is expected above background. For the LHC data set, ambiguities arising from multiple solutions still make it difficult to pin down the value of $\langle J\rangle$. We have quoted the error on $\langle J\rangle$ obtained when we resolve this ambiguity in favor of the correct solution by removing the subsidiary peaks in the likelihood distributions for $\sigma v$ at high cross section values. The variances are still quite large. These ambiguities are resolved by the ILC data, and for those cases we give the fractional error on $\langle J\rangle$ computed from the full scan data. Thus, at LCC2 and at LCC4, we find that $\langle J\rangle$ is given to 30-40\% accuracy. These accuracies should be compared to the current astrophysical estimates of $\langle J\rangle$ at the galactic center, which range over many orders of magnitude. A measurement of the $\langle J\rangle$ correct to the level we have shown, and even the model-independent upper limit on $\langle J\rangle$, would represent a major improvement in our knowledge.

For our model dark matter clump in the galactic halo, the situation is somewhat better. The background still dominates for LCC1, but not in the other cases. For the LHC data sets, the problem of ambiguities in the solution is still present, and again we restrict our estimates to MCMC points in the peak corresponding to the correct solution. But, using the ILC data, we will find quantitative measurements of $\langle J\rangle$. At LCC2, we can achieve a $20 \%$ measurement already with the $500 \mathrm{GeV}$ data. At all of the benchmark points except for LCC1, the data from the $1000 \mathrm{GeV}$ stage determines $\langle J\rangle$ an accuracy of $20 \%$ or better.

For the local flux of neutralino dark matter, we find a similar picture. In all cases except for LCC2, the LHC data set provides only a weak determination of the relevant WIMP cross section. We can at best quote an upper limit on the cross section and thus a lower limit on the local flux. For the LHC data at LCC2, we have quoted a measurement error after removing the peak arising from the incorrect solutions. The data from the $500 \mathrm{GeV}$ ILC constrains the direct detection cross sections to some 
extent, and for these entries we have quoted the fractional error, which is however large in all cases. The measurements at the $1000 \mathrm{GeV}$ ILC finally fix the mass of the heavy Higgs bosons and other spectroscopic parameters involved in the most important contributions to the direct detection cross section. From this data, the local flux can be determined to 20-30\% accuracy for all four benchmark models. Our current knowledge of the galactic halo constrains this flux only to a factor of 2 , and even then only if we assume that the halo has a smooth distribution both in position and in momentum space. Here too, precise microscopic information can have a large impact.

In all three examples, what would be determined would be the density or flux of that component of dark matter corresponding to the WIMP observed in collider experiments. Other possible components of dark matter such as axions or very heavy weakly-interacting particles give negligible signals in direct detection experiments and experiments on dark matter annihilation. Thus, first of all, the direct and indirect detection experiments would demonstrate concretely that the WIMP seen in particle physics is present in the structure of the galaxy. At the next stage, the consistency of the overall picture that results from these experiments could give additional insight, beyond what is gained from analysis of the overall relic density, on the broad question of whether the observed WIMP is the dominant component of dark matter.

\subsection{LHC and astrophysical measurements}

Up to this point, we have been discussing the comparison to astrophysical data of the full set of results that we will obtain from the next-generation colliders. It will take some time, of course, for the collider data to become available. The LHC experiments will begin in just another year. The $500 \mathrm{GeV}$ stage of ILC may begin within ten years from now. The $1000 \mathrm{GeV}$ stage of the ILC would be an upgrade to the basic facility and would produce data, at the earliest, at the end of the next decade. Astrophysical dark matter experiments are also spaced out in time through the next decade. It is interesting, then, to look at the implications of colliders in terms of this timeline and see what results can be expected at each stage. In this and the next two sections, we will discuss some aspects of this evolution, emphasizing the the new information that we will obtain as each collider presents its results.

We begin with the situation as it might appear in 2012, when we have the results of the LHC experiments and the first positive results from direct detection experiments and searches for WIMP annihilation.

At this stage, it should already be possible to compare three observed masses relevant to dark matter: (1) the mass of the escaping neutral particle produced at the LHC in missing energy events, (2) the mass of the directly detected dark matter 
particle obtained from the recoil energy spectrum, and (3) the mass of the annihilating dark matter particle, obtained from the endpoint of the gamma ray spectrum. In the best case, all three masses should be determined to better than $20 \%$ accuracy. Their agreement will provide a nontrivial, model-independent test that the particle being produced at the LHC is indeed a dominant component of cosmic dark matter.

It will be very difficult to go further in a truly model-independent analysis. As we have discussed in Section 2.3, it will be very difficult from the LHC data alone to narrow the possible explanations of missing-energy events and new particles to a single model. Nevertheless, we might proceed by assuming a particular model (for example, supersymmetry or even a restricted model of supersymmetry) and examining its consequences for astrophysics.

In some scenarios (for example, the benchmark points LCC1 and SPS1a' discussed in Section 4), we would be able to combine the assumption that the new physics is supersymmetry with the detailed spectroscopy measurements available from the LHC to give a quite accurate prediction of the WIMP relic density. In this situation, it is tempting to assume that the observed WIMP is the sole component of dark matter, fix the relic density to the value measured from the cosmic microwave background, and make higher-precision predictions for the supersymmetry mass spectrum. In addition, because the heavy Higgs bosons typically give the dominant contribution to the spin-independent direct detection cross section, one can assume that the local flux of dark matter is near its nominal value and use the direct detection rate to fix the heavy Higgs boson mass. In both cases, the later stages of the collider physics program on supersymmetry spectroscopy will test these predictions and thus confirm or refute the astrophysical assumptions.

In most scenarios, however, it is not possible to derive a definite prediction for the WIMP relic density from the LHC data even if supersymmetry is assumed to be the underlying theory. The precise point in supersymmetry parameter space might not be determined uniquely from the data, or the data might not select sufficiently precisely the special mechanism of neutralino annihilation. We have seen examples of the first difficulty at LCC2 and of the second at LCC3 and LCC4. In these cases, the LHC would give us only a first tantalizing glimpse of the particle physics origin of dark matter, leaving many questions to be resolved by the ILC experiments.

We have also noted a circumstance in which qualitative information from the LHC can be bootstrapped into quantitative information for astrophysics. The particle physics cross section needed for the interpretation of gamma ray data must be close to the value (2) if the observed WIMP is the dominant component of dark matter and if the relic density of WIMPs is set primarily by WIMP annihilation in the S-wave without coannihilation. The LHC data could point to a qualitative scenario (one similar to LCC2, for example) in which S-wave annihilation would be expected. We 
would then have a quantitative estimate of the annihilation cross section that could be used to analyze astrophysical gamma ray spectra.

\subsection{ILC at $500 \mathrm{GeV}$}

In any scenario, the estimates of the neutralino properties from the LHC would be dramatically improved when the neutralino and the other light particles in its sector are observed in $e^{+} e^{-}$annihilation.

First of all, the measurements of $e^{+} e^{-}$annihilation cross sections and angular distributions will give the spins and Standard Model quantum numbers of the lightest states in the new particle sector, allowing definite identification of the model that is giving rise to the stable WIMP. This identification is a prerequisite for any 'modelindependent' estimation of the WIMP cross sections.

Second, measurements in $e^{+} e^{-}$annihilation can improve the accuracy on the WIMP mass from $5-10 \%$ at the LHC to a fraction of a percent. The value of $\Omega_{\chi} h^{2}$ is directly correlated with the WIMP mass in all of our models, so this is crucial information for obtaining an accurate prediction of the relic density.

Third, measurements in $e^{+} e^{-}$annihilation can identify all light partners of the WIMP with electromagnetic or weak charge, including some (e.g., in supersymmetry, the charginos and the staus) that are difficult to study at hadron colliders. The masses of these particles would also be measured to a fraction of a percent.

Fourth, measurements of production cross sections in $e^{+} e^{-}$annihilation give direct sensitivity to the mixing angles that define the mass eigenstates of the new particles. In supersymmetry, the cross sections for chargino and neutralino pair production fix the gaugino-Higgsino mixing angles, and the cross section for stau pair production fix the stau mixing angles. At a linear collider, the cross sections with polarized beams can be measured, and the individual cross sections from left- and right-handed polarized electrons provide complementary information. We have seen one special case (at LCC1) where the measurement of a ratio of branching ratios at the LHC can substitute some of this information. But the technique of extracting these angles from polarized cross section measurements is general and much more effective.

Finally, the information from spectra and cross sections obtained in $e^{+} e^{-}$annihilation allows one to resolve ambiguities that arise in imposing the constraints from the LHC data. Here we refer both to the question of multiple solutions to the various eigenstate mixing problems and to the question of whether special situations such as a Higgs boson resonance or coannihilation are present. In some cases, as we have discussed, these predictions can be sharpened by adding constraints from the relic density or from direct detection rates. 
Looking back at the tables, we see that, in almost all cases, we do not obtain precise determinations of the basic WIMP cross sections until we have data from $e^{+} e^{-}$experiments.

In this paper, we have intentionally chosen models in which the lightest states of the new particle spectrum can be explored at the ILC at a center of mass energy of $500 \mathrm{GeV}$. This is our expectation, based on the idea that models of WIMPs arise naturally from models of electroweak symmetry breaking. We will learn very soon from the LHC whether this assumption is justified. If the spectrum of new particles associated with the WIMP is out of reach of the $500 \mathrm{GeV}$ ILC, we will still need $e^{+} e^{-}$ data to understand the dark matter problem. We will just have to wait longer to obtain it.

\subsection{ILC at $1000 \mathrm{GeV}$}

Although in all of our models the ILC at $500 \mathrm{GeV}$ gives a great improvement in the information available from colliders, we always found an advantage in doing additional $e^{+} e^{-}$experiments at higher energy. In all of the models except LCC2, this higher-energy running was particularly important for one specific reason: The WIMP cross sections are sensitive to the masses and couplings of the heavy Higgs bosons, and we needed accurate values of the Higgs boson masses and the mixing angle $\tan \beta$ to determine these cross section accurately. The spin-independent direct detection cross section, in particular, is typically dominated by $t$-channel Higgs boson exchange. If the heavy Higgs bosons are not seen directly, there is no strong prediction for this cross section; once these Higgs bosons are seen, there is suddenly a precise determination from the collider data.

In supersymmetric models, the heavy Higgs bosons are typically heavier than the lightest superpartners. In this range of parameters, they are pair-produced in the process $e^{+} e^{-} \rightarrow H^{0} A^{0}$. In our study, it was important for $e^{+} e^{-}$experiments to reach the threshold for this process in order to complete the set of experiments needed to predict the WIMP properties.

\subsection{Conclusions}

Finally, we return to the larger picture. In this paper, we have shown that the experimental results from the hadron and $e^{+} e^{-}$colliders of the next generation can be used to determine the basic particle physics cross sections of WIMP dark matter particles. Using this information, we will be able to test whether particles seen at high-energy accelerators make up cosmic dark matter. If this is so, we can apply the cross sections determined from collider data to astrophysical dark matter experiments 
and use them to study in a very general way the distribution of dark matter in the galaxy.

We expect that this study will form one of the major themes of experimental highenergy physics and experimental astrophysics over the next ten to fifteen years. It will lead us to learn much more about the structure of the galaxy and of the universe, and also about the underlying structure of the elementary particles and their laws. To carry out the full program will take persistence and it will require collaboration across the field of physics. But if we can see the goal, we can reach it.

\section{A Markov Chain Monte Carlo}

In this appendix we detail the Markov Chain Monte Carlo technique used to explore the $D=24$ dimensional parameter space described in this paper. We then describe tests of the Markov chains' convergence.

\section{A.1 Adaptive Metropolis-Hastings algorithm}

The Metropolis-Hastings algorithm is simple. Consider a point $\vec{p}_{i}$ in the parameter space. From this point, a new point $\vec{q}$ is proposed, with probability density $P\left(\vec{q}, \vec{p}_{i}\right)$. Note that the simple algorithm requires that that the density $P$ be symmetric in its arguments. If the likelihood of the proposed point is larger than that at the current point, $\mathcal{L}(\vec{q}) \geq \mathcal{L}\left(\vec{p}_{i}\right)$, then set $\vec{p}_{i+1}=\vec{q}$. If the likelihood at the proposed point is lower, set $\vec{p}_{i+1}=\vec{q}$ with probability $\mathcal{L}(\vec{q}) / \mathcal{L}\left(\vec{p}_{i}\right)$, otherwise set $\vec{p}_{i+1}=\vec{p}_{i}$. The set of points $\left\{\vec{p}_{i}\right\}$ then converges to the correct target distribution, independent of the proposal density $P$. The difficulty in implementing this algorithm lies entirely in choosing a proposal $P$ which allows an efficient exploration of the target distribution.

We can use the covariance matrix to construct an efficient proposal. From a sample set of $N$ points $\vec{p}_{i}$, we construct the mean and covariance matrix,

$$
\begin{aligned}
\vec{\mu} & =\frac{1}{N} \sum_{i=1}^{N} \vec{p}_{i}, \\
\mathbf{C} & =\frac{1}{N} \sum_{i=1}^{N}\left(\vec{p}_{i}-\vec{\mu}\right)\left(\vec{p}_{i}-\vec{\mu}\right)^{T} .
\end{aligned}
$$

Note that for $\mathbf{C}$ to be a positive definite matrix, $N$ must be at least $D+1$. This matrix is essentially the variance; it can be thought of as $\boldsymbol{\sigma}^{2}$. If the sample set reasonably covers the region of high likelihood, then the shape of the region, complete with 
degenerate directions, etc., is encoded in C. Is is natural to use a Gaussian proposal distribution based on the covariance matrix and scaled by an efficiency factor $f$. The proposal is $\vec{q}=\overrightarrow{p_{i}}+\vec{y}$, where $\vec{y}$ is distributed as

$$
P(\vec{y})=\frac{1}{\sqrt{\left(2 \pi f^{2}\right)^{D} \operatorname{det} \mathbf{C}}} \exp \left(-\frac{1}{2 f^{2}} \vec{y}^{T} \mathbf{C}^{-1} \vec{y}\right) .
$$

This can be implemented by choosing a vector $\vec{x}$ where each element has a Gaussian distribution with zero mean and unit variance and taking $\vec{y}=f \sqrt{\mathbf{C}} \vec{x}$. By $\sqrt{\mathbf{C}}$ we mean any matrix $\mathbf{L}$ such that $\mathbf{L} \mathbf{L}^{T}=\mathbf{C}$. It is convenient to take $\mathbf{L}$ to be lower triangular: this is the Cholesky decomposition of $\mathbf{C}$. It can be shown that for a Gaussian target distribution, the most efficient step is $f=2.381 / \sqrt{D}$ in the limit of large $D$. This prefactor is fairly optimal even for $D=1$ [77]. Alternatively, we can use a Cauchy-Lorentz proposal distribution. This has the advantage of occasionally allowing very long steps, though for a Gaussian target distribution, it is less efficient. The Cauchy-Lorentz distribution in $D$ dimensions is given by

$$
P(\vec{y})=\frac{(2 / f)^{D} \Gamma\left(\frac{D+1}{2}\right)}{\sqrt{\pi^{D+1} \operatorname{det} \mathbf{C}}}\left(1+\frac{4}{f^{2}} \vec{y}^{T} \mathbf{C}^{-1} \vec{y}\right)^{-(D+1) / 2}
$$

This can be implemented analogously by taking $\vec{x}$ to have a Cauchy-Lorentz distribution with a unit full-width at half maximum as follows,

$$
x_{i}=\frac{t_{i}}{2 \sqrt{i}}\left(1+4 \sum_{j=1}^{i-1} x_{j}^{2}\right)^{1 / 2}, i \in\{1 \ldots D\}
$$

where $t_{i}$ has a $t$-distribution with $i$ degrees of freedom. As before, $\vec{y}=f \sqrt{\mathbf{C}} \vec{x}$.

The Metropolis-Hastings algorithm can be made adaptive, by updating the matrix C. A key consequence of this is that the proposal distribution is no longer symmetric, which must be accounted for. We chose a method that uses $N \geq D+1$ Markov chains in parallel. The covariance matrix used in the proposal is constructed from the current point of each chain. At each step, a chain is chosen at random, and a proposal is made for that chain. If accepted, the one chain is advanced, and now of course the covariance matrix changes, $\mathbf{C} \rightarrow \mathbf{C}^{\prime}$. This means that the probability density to return to the previous state is not the same as the probability density to arrive at the current state from the previous state. If this is not corrected, detailed balance is violated. As a shorthand, call the two probability densities $P(\mathbf{C})$ and $P\left(\mathbf{C}^{\prime}\right)$. Detailed balance is restored with the following acceptance probability,

$$
P(\text { accept })=\min \left[1, \frac{P\left(\mathbf{C}^{\prime}\right) \mathcal{L}(\vec{q})}{P(\mathbf{C}) \mathcal{L}\left(\vec{p}_{i}\right)}\right] .
$$


The step $\vec{x}^{\prime}$ required to return is different than the step $\vec{x}$, but both are unit-Gaussian distributed. For the Gaussian case, it is easy to see that

$$
\begin{aligned}
\frac{P\left(\mathbf{C}^{\prime}\right)}{P(\mathbf{C})} & =\sqrt{\frac{\operatorname{det} \mathbf{C}}{\operatorname{det} \mathbf{C}^{\prime}}} \exp \left[-\frac{1}{2}\left(x^{\prime 2}-x^{2}\right)\right] \\
& =\sqrt{\frac{\operatorname{det} \mathbf{C}}{\operatorname{det} \mathbf{C}^{\prime}}} \exp \left[-\frac{1}{2 f^{2}} \vec{y}^{T}\left(\mathbf{C}^{\prime-1}-\mathbf{C}^{-1}\right) \vec{y}\right]
\end{aligned}
$$

We now need to know $\mathbf{C}^{\prime}$ and its inverse and determinant. Assume that the point $\vec{p}_{j}$ is the one to be updated, $\vec{p}_{j} \rightarrow \vec{p}_{j}+\vec{y}$, we find

$$
\begin{aligned}
\vec{\mu}^{\prime} & =\vec{\mu}+\frac{1}{N} \vec{y}, \\
\mathbf{C}^{\prime} & =\frac{1}{N} \sum_{i=1}^{N}\left[\vec{p}_{i}-\vec{\mu}+\left(\delta_{i j}-\frac{1}{N}\right) \vec{y}\right]\left[\vec{p}_{i}-\vec{\mu}+\left(\delta_{i j}-\frac{1}{N}\right) \vec{y}\right]^{T}, \\
& =\mathbf{C}+\frac{1}{N}\left(\vec{p}_{j}-\vec{\mu}\right) \vec{y}^{T}+\frac{1}{N} \vec{y}\left(\vec{p}_{j}-\vec{\mu}\right)^{T}+\frac{N-1}{N^{2}} \vec{y} \vec{y}^{T} .
\end{aligned}
$$

The covariance matrix has been adjusted by the addition of dyad products, and only two of them (collecting terms in $\vec{y}^{T}$ ). It is possible to invert such a matrix analytically if the inverse of the base matrix is known.

$$
\begin{aligned}
\mathbf{C}^{\prime} & =\mathbf{C}+\sum_{i} \vec{a}_{i} \vec{b}_{i}^{T} \\
\lambda_{i j} & =\vec{b}_{i}^{T} \mathbf{C}^{-1} \vec{a}_{j} \\
\mathbf{C}^{\prime-1} & =\mathbf{C}^{-1}-\sum_{i, j}(\mathbf{1}+\boldsymbol{\lambda})_{i j}^{-1} \mathbf{C}^{-1} \vec{a}_{i} \vec{b}_{j}^{T} \mathbf{C}^{-1}, \\
\operatorname{det} \mathbf{C}^{\prime} & =\operatorname{det} \mathbf{C} \operatorname{det}(\mathbf{1}+\boldsymbol{\lambda}) .
\end{aligned}
$$

There are only two dyads in our case, thus the matrix inversion in (33) is trivial. In this way, we do not need to perform a $D \times D$ matrix inversion for each proposal. If the proposal is accepted, we do need to recompute the Cholesky decomposition as there is no such formula to update it. This does save time, as we typically want acceptance probabilities around 25\%. Notice here that both probability densities $P(\mathbf{C})$ and $P\left(\mathbf{C}^{\prime}\right)$ have an implicit factor of $1 / N$ insuring that it is in fact chain $j$ that is being updated. In evaluating (27), we need $\mathbf{C}^{-1}=\mathbf{L}^{-1, T} \mathbf{L}^{-1}$. Since $\mathbf{L}$ is lower triangular, its inverse multiplied by a vector is trivially obtained by back-substitution. Then (34) is easily verified for the case of adding a single dyad. The result for an arbitrary number of dyads follows inductively: if true for $n-1$ dyads, the case of $n$ dyads is shown to be the Laplace expansion for the $n \times n$ determinant. 
For a Cauchy-Lorentz proposal, the correction factor is quite similar. Using the results of the previous paragraph,

$$
x^{2}-x^{2}=\Delta x^{2}=-\frac{1}{f^{2}}(\mathbf{1}+\boldsymbol{\lambda})_{i j}^{-1} \vec{y}^{T} \mathbf{C}^{-1} \vec{a}_{i} \vec{b}_{j} \mathbf{C}^{-1} \vec{y},
$$

we find

$$
\frac{P\left(\mathbf{C}^{\prime}\right)}{P(\mathbf{C})}=\frac{1}{\sqrt{\operatorname{det}(\mathbf{1}+\boldsymbol{\lambda})}}\left(1+\frac{4 \Delta x^{2}}{1+4 x^{2}}\right)^{-(D+1) / 2}
$$

This efficiency of this adaptive proposal relies on the fact that the target distribution is Gaussian. If it is non-Gaussian, which is always the case for the distributions studied in this paper, the efficiency may be different. We take a stepsize $f=2.381 \epsilon / \sqrt{D}$, where $\epsilon=1$ is most efficient in the Gaussian case. For the various cases studied, we used efficiencies $\epsilon \in[0.15,0.5]$ as required to have acceptance probabilities that were not too small (above $5 \%$ ).

\section{A.2 Exploring the distributions}

For each of the 12 cases (four LCC benchmark points, three colliders), we have run Markov Chains as follows. Our fiducial runs have $N=25$ chains in parallel, the minimal number that gives a positive definite covariance matrix. The total number of samples taken is $4 \times 10^{6}$, so roughly $1.6 \times 10^{5}$ per chain. Starting at the beginning, some fraction of each chain, up to $10 \%$, is used for "burn-in" to find the region of acceptable likelihood. For the first half of the burn-in period, we apply a "cooling" technique where the "temperature" is gradually lowered to the correct value. This means that we take the likelihood function to be $\mathcal{L}^{\lambda}$. We update $\lambda=1 / T$ geometrically by $\lambda_{i}=\lambda_{0}^{1-i / n}$, where $n$ is the total number of burn-in points, and we take $\lambda_{0}=0.01$. Note that the current temperature is used for both likelihoods in the acceptance probability.

\section{A.3 Thinning the chains}

We do not compute relic density at every point due to the computational expense. The chains are correlated at short distances so this is not even necessary to achieve good statistics. We instead thin the chains by some factor, usually taken to be $t=50$. This means we construct a new chain from every 50th point of the current chain. We only compute relic density for the points of the thinned chains. 


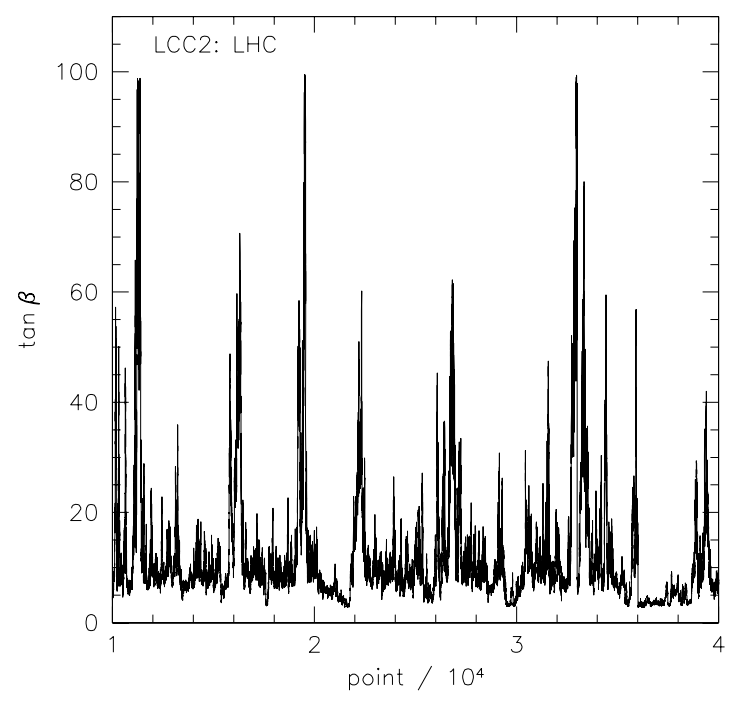

Figure 59: Section of the Markov chain for $\tan \beta$ at LCC2-LHC. The repeated brief excursions to large values are clearly seen.

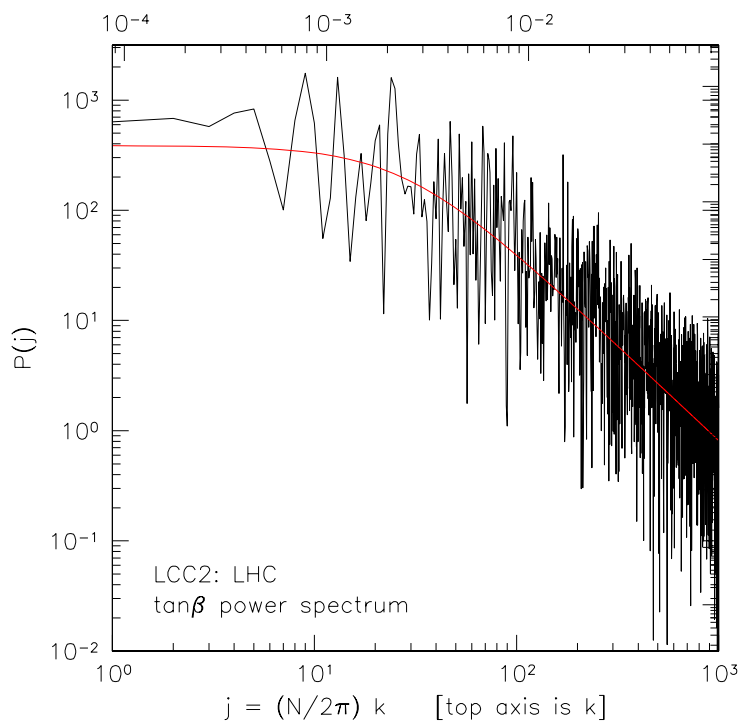

Figure 60: Fourier transform of the Markov chain for $\tan \beta$ at LCC2-LHC. A constant rolling into a power law fit [77] is shown. This illustrates the white noise (flat) spectrum for low $k$ indicating decorrelation at large distances in the chain and the random walk $\left(k^{-2}\right)$ spectrum at short distances (large $k$ ). 


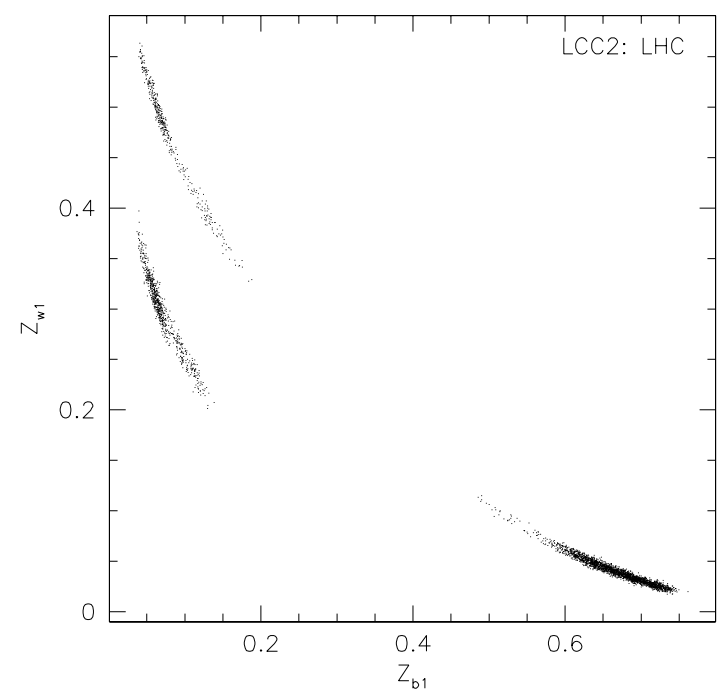

Figure 61: Illustration of MCMC bridging the gap between islands in parameter space. These are solutions for LCC2 with LHC data, as in Fig. 25. The true solution has the largest bino fraction $Z_{b 1}$.

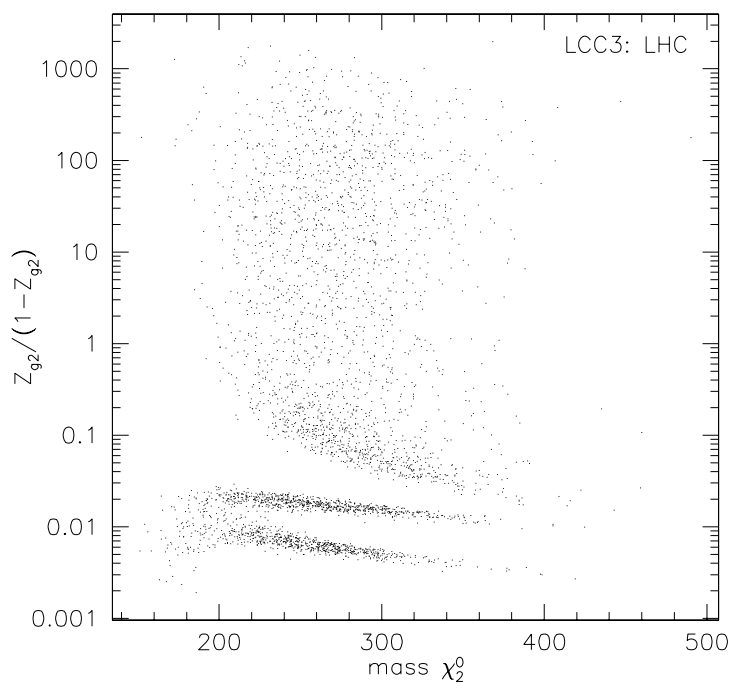

Figure 62: Illustration of MCMC exploring very different regions of parameter space. These regions are actually connected, as shown in Fig. 36, but allow vastly different properties for $\chi_{2}^{0}$, as shown here. 


\section{A.4 Convergence test}

We apply the technique of [77] to determine whether the Markov Chains have converged. In a given run, the $N$ chains are concatenated (after removing the burn-in periods) to construct one long chain of length $N_{\text {tot }}$. For each of the 24 parameters, we normalize to unit variance and take the Fourier transform. We then fit the power spectrum $P(k)=\left|a_{j}\right|^{2}$, where the $a_{j}$ are the Fourier components (with $j$ ranging between $-N_{\text {tot }} / 2$ and $N_{\text {tot }} / 2$ ) and $k=2 \pi j / N_{\text {tot }}$. If the elements of the chain are uncorrelated at large separation, then for small $k$ (long distance in the chain), we expect to see a white noise spectrum, that is, a power spectrum that is approximately constant. At short distances in the chain (large $k$ ) we expect the power spectrum to behave like $k^{-2}$, the random walk spectrum. Following [77], we require that the break between these two behaviors occurs beyond $j=20$. When this is true, the fit to $P(0)$ can be trusted, and $r=P(0) / N_{\text {tot }}$ indicates the fractional error made in the variance of the parameter. We require $r<0.01$, meaning at most $10 \%$ errors made in the error of a given parameter, based on the Markov chain.

The power spectrum test can be applied either to the full chain or the thinned chain. The results are insensitive as long as the scales removed are in the random walk regime. For chains of length $N_{\text {tot }}$, thinning by a factor of $t$ has little effect unless the turnover is beyond $j=N_{\text {tot }} / t$. We typically find $j$ values at turnover of $20-200$, so this is not an issue.

\section{B Disconnected families of supersymmetry parameters}

In this appendix we describe the conditions where multiple discrete regions of parameter space would be consistent with collider measurements. Such ambiguities occur for all of the benchmark points under study. The simplest alternate solutions involve only changing the signs of $\mu$ and $m_{1}$. This four-fold degeneracy is evident in every model given only LHC data. Since these signs are relevant to the mixing parameters of the neutralinos and charginos, it is essential to measure the mixed pair

production cross sections at the ILC, e.g., $e^{+} e^{-} \rightarrow \widetilde{\chi}_{1}^{0} \widetilde{\chi}_{2}^{0}$. There are more subtle degeneracies as well, as we discuss in the following sections.

\section{B.1 Benchmark point LCC1}

At the LHC, three neutralinos are visible: the lightest (bino) at $95.5 \mathrm{GeV}$, the next lightest (wino) at $181.6 \mathrm{GeV}$, and the heaviest (heavier Higgsino) at $375.6 \mathrm{GeV}$. No chargino is seen, implying a mass $>125 \mathrm{GeV}$. Assuming that the identities of the 
neutralinos are unknown at the LHC, it is possible that the values of the parameters $|\mu|$ and $m_{2}$ are exchanged. This implies that the second and third neutralinos are Higgsinos, and the heaviest is mostly wino. This solution gives an acceptable mass spectrum for the LHC errors. In addition, there is the four-fold degeneracy due to the uncertain signs of $\mu$ and $m_{1}$. There are thus 8 solutions for LHC data. Notice that we can be confident that the lightest neutralino is mostly bino-like; its mass is $95 \mathrm{GeV}$, and a chargino at this mass would be visible.

The ILC-500 completely resolves the degeneracies of this model. The identities of $\widetilde{\chi}_{2}^{0}$ and $\tilde{\chi}_{1}^{+}$as wino-like are determined by the pair production cross sections, and the sign ambiguities are completely resolved by the $\tilde{\chi}_{1}^{0} \widetilde{\chi}_{2}^{0}$ production cross section: changing the signs has a large effect on the (small) bino fraction of $\widetilde{\chi}_{2}^{0}$.

At this point, the 8 islands are very well separated in parameter space. For the LHC, we have shown the results of the correct island only. Including the other solutions would not make a large difference, as they only affect the subdominant wino and Higgsino admixtures in the lightest neutralino.

\section{B.2 Benchmark point LCC2}

The three lightest neutralinos are visible at the LHC, while there is only the usual limit of $125 \mathrm{GeV}$ on the lightest chargino mass. All of the neutralinos are quite mixed in composition, thus the permutation ambiguities in $m_{1}, m_{2}, \mu$ are somewhat ambiguous. As shown in Fig. 61, there are essentially three solutions for positive $\mu$ and $m_{1}$. When plotted as islands in neutralino composition, the solutions are clearly separated. The incorrect solutions have the lightest neutralino being mostly wino-like or Higgsino-like rather than mostly bino-like. In fact, the wino and Higgsino cases have two peaks, not well separated, as seen in Fig. 25. There is an additional bino solution as well (not shown), with $M_{2}$ larger than $1 \mathrm{TeV}$, but its properties are similar to the proper bino solution, except that the wino fraction is very much smaller.

The ILC-500 removes the degeneracies. The $\widetilde{\chi}_{2}^{0} \widetilde{\chi}_{3}^{0}$ cross section identifies the neutralinos and also their mixing angles, thus the negative $\mu$ solution at least is gone. The ILC-1000 allows no incorrect solution.

At this point only, the disjoint islands at positive $\mu$ and $m_{1}$ are close together in parameter space. Our LHC results show all islands with the correct signs. During the cooling and burn-in period, Markov chains find an island at random, and remain there for the remainder of the run. We find that burn-in is slow enough that the number of chains that finds a solution is proportional to the likelihood of the solution. We have verified this with a "stepping stone" technique, where the space between the islands, normally having vanishing likelihood, is assigned a small likelihood. Markov chains can now step away from an island, into this "sea", and thus travel back and 
forth between islands. Points in the "sea" can be removed at the end with importance sampling. We have verified the relative weights of the islands with just such a simulation.

\section{B.3 Benchmark points LCC3 and LCC4}

Benchmark points LCC3 and LCC4 have similar structure for discrete solutions, thus we discuss them together. Both have the two lightest neutralinos visible at the LHC. Assuming that none have been missed between the two, the " $F$ " structure in the $m_{1}$ vs. $\left(m_{2}\right.$ or $\left.\mu\right)$ plot appears naturally. This is understood physically as follows. Given the large mass splitting between the two visible neutralinos, and the assumption that none have been missed, the lightest neutralino is either bino or wino, and the heavier of the two visible neutralinos can be anything. As all neutralinos at these benchmark points are heavier than $125 \mathrm{GeV}$, the non-observation of charginos gives no additional information. The "F" structure is repeated for each of the four sign combinations for $m_{1}$ and $\mu$. In Fig. 62 we illustrate an interesting effect of the "F" structure, in that there are three possible solutions to the composition of the second lightest neutralino.

For each of these points, ILC-500 data would collapse the "F" structure somewhat, but the sign ambiguities remain. ILC-1000 is required to remove the last ambiguities, with the neutralino and chargino pair production cross sections.

Unfortunately, the sign ambiguity in $\mu$ remains for point LCC4, even taking ILC1000 data into account. However, since this point has large $\tan \beta$, its correction to the muon $g-2$ is large, and has a sign equal to the sign of $\mu$. The BNL measurement [94] rules out the negative $\mu$ solution, even with a very conservative constraint allowing the union of the $3 \sigma$ regions from the $e^{+} e^{-}$and $\tau$ decay evaluations of the hadronic vacuum polarization $[95,96]$.

For these two points, we have only illustrated the correct signs. The " $F$ " patterns are too far apart in parameter space for easy exploration by single Markov chains.

\section{ACKNOWLEDGEMENTS}

Our perspective on dark matter has been shaped by discussions with many people over the past few years. We are particularly grateful to Genevieve Belanger, Andreas Birkedal, Jonathan Feng, Paolo Gondolo, Konstantin Matchev, and Mark Trodden for sharing their insights. We thank Mihoko Nojiri and Giacomo Polesello for an helpful correspondence. EAB thanks Richard Schnee, Blas Cabrera and Dan Akerib for many 
useful discussions on the capabilities of direct detection experiments, and Larry Wai and Tune Kamae for useful discussions relating to gamma ray detection. MEP thanks Jim Alexander, Richard Gray, Bhaskar Dutta, and Teruki Kamon for discussions of their ILC simulation results. He is also grateful to Ee Hou Yong and Wu-Yen Chuang for educating him about neutralino dark matter. The work of MB was supported by the US Department of Energy under Contract No. DE-AC02-05CH11231 and used resources of the National Energy Research Scientific Computing Center, supported by Contract No. DE-AC03-76SF0098. The work of EAB, MEP, and TW was supported by the US Department of Energy under Contract No. DE-AC02-76SF00515.

\section{References}

[1] O. Lahav and A. Liddle, in S. Eidelman, et al., Review of Particle Properties, Phys. Lett. B592, 1 (2004); http://pdg.1bl.gov/.

[2] G. Jungman, M. Kamionkowski and K. Griest, Phys. Rept. 267, 195 (1996) [arXiv:hep-ph/9506380].

[3] G. Bertone, D. Hooper and J. Silk, Phys. Rept. 405, 279 (2005) [arXiv:hep$\mathrm{ph} / 0404175]$.

[4] W. Hu, R. Barkana and A. Gruzinov, Phys. Rev. Lett. 85, 1158 (2000) [arXiv:astro-ph/0003365].

[5] B. Moore, Astrophys. J. 413, L93 (1993) [arXiv:astro-ph/9306004].

[6] D. N. Spergel et al. [WMAP Collaboration], Astrophys. J. Suppl. 148, 175 (2003) [arXiv:astro-ph/0302209].

[7] J. R. Bond, G. Efstathiou and M. Tegmark, Mon. Not. Roy. Astron. Soc. 291, L33 (1997) [arXiv:astro-ph/9702100].

[8] F. E. Paige, S. D. Protopescu, H. Baer and X. Tata, arXiv:hep-ph/0312045.

[9] P. Gondolo, J. Edsjo, P. Ullio, L. Bergstrom, M. Schelke and E. A. Baltz, JCAP 0407, 008 (2004) [arXiv:astro-ph/0406204], New Astron. Rev. 49, 149 (2005).

[10] B. C. Allanach, G. Belanger, F. Boudjema and A. Pukhov, JHEP 0412, 020 (2004) [arXiv:hep-ph/0410091].

[11] M. M. Nojiri, G. Polesello and D. R. Tovey, arXiv:hep-ph/0512204.

[12] M. Battaglia and M. E. Peskin, arXiv:hep-ph/0509135. 
[13] R. J. Scherrer and M. S. Turner, Phys. Rev. D 33, 1585 (1986) [Erratum-ibid. D 34, 3263 (1986)].

[14] H. Pagels and J. R. Primack, Phys. Rev. Lett. 48, 223 (1982).

[15] S. Weinberg, Phys. Rev. Lett. 48, 1303 (1982).

[16] S. Weinberg, Phys. Rev. Lett. 50, 387 (1983).

[17] H. Goldberg, Phys. Rev. Lett. 50, 1419 (1983).

[18] J. R. Ellis, D. V. Nanopoulos and K. Tamvakis, Phys. Lett. B 121, 123 (1983).

[19] H. C. Cheng, J. L. Feng and K. T. Matchev, Phys. Rev. Lett. 89, 211301 (2002) [arXiv:hep-ph/0207125].

[20] G. Servant and T. M. P. Tait, Nucl. Phys. B 650, 391 (2003) [arXiv:hep$\mathrm{ph} / 0206071]$.

[21] K. Agashe and G. Servant, Phys. Rev. Lett. 93, 231805 (2004) [arXiv:hep$\mathrm{ph} / 0403143]$.

[22] E. Katz, J. y. Lee, A. E. Nelson and D. G. E. Walker, arXiv:hep-ph/0312287.

[23] H. C. Cheng and I. Low, JHEP 0309, 051 (2003) [arXiv:hep-ph/0308199].

[24] S. J. Asztalos et al., Astrophys. J. 571, L27 (2002) [arXiv:astro-ph/0104200]; Phys. Rev. D 69, 011101 (2004) [arXiv:astro-ph/0310042].

[25] D. R. Tovey, Eur. Phys. J. direct C 4, N4 (2002).

[26] M. Battaglia, A. Datta, A. De Roeck, K. Kong and K. T. Matchev, JHEP 0507, 033 (2005) [arXiv:hep-ph/0502041]; arXiv:hep-ph/0507284.

[27] A. Datta, K. Kong and K. T. Matchev, arXiv:hep-ph/0509246.

[28] J. M. Smillie and B. R. Webber, arXiv:hep-ph/0507170.

[29] J. L. Feng, A. Rajaraman and F. Takayama, Phys. Rev. Lett. 91, 011302 (2003) [arXiv:hep-ph/0302215].

[30] J. L. Feng and B. T. Smith, Phys. Rev. D 71, 015004 (2005) [Erratum-ibid. D 71, 0109904 (2005)] [arXiv:hep-ph/0409278].

[31] J. L. Feng, A. Rajaraman, B. T. Smith, S. Su and F. Takayama, arXiv:hep$\mathrm{ph} / 0410178$. 
[32] T. Moroi and L. Randall, Nucl. Phys. B 570, 455 (2000) [arXiv:hep-ph/9906527].

[33] R. Kitano and I. Low, Phys. Rev. D 71, 023510 (2005) [arXiv:hep-ph/0411133]; R. Kitano and Y. Nomura, arXiv:hep-ph/0509221.

[34] K. A. Olive, G. Steigman and T. P. Walker, Phys. Rept. 333, 389 (2000) [arXiv:astro-ph/9905320].

[35] E. I. Gates, G. Gyuk and M. S. Turner, Phys. Rev. D 53 (1996) 4138 [arXiv:astroph/9508071], E. Gates, G. Gyuk and M. S. Turner, arXiv:astro-ph/9704253.

[36] A. K. Drukier, K. Freese and D. N. Spergel, Phys. Rev. D 33, 3495 (1986).

[37] D. Stiff, L. M. Widrow and J. Frieman, Phys. Rev. D 64, 083516 (2001) [arXiv:astro-ph/0106048]; D. Stiff and L. M. Widrow, Phys. Rev. Lett. 90, 211301 (2003) [arXiv:astro-ph/0301301].

[38] P. Sikivie and J. R. Ipser, Phys. Lett. B 291, 288 (1992); P. Sikivie, Phys. Lett. B 432, 139 (1998) [arXiv:astro-ph/9705038].

[39] J. Diemand, B. Moore and J. Stadel, Nature 433, 389 (2005) [arXiv:astro$\mathrm{ph} / 0501589]$.

[40] K. Freese, P. Gondolo and H. J. Newberg, Phys. Rev. D 71, 043516 (2005) [arXiv:astro-ph/0309279]; K. Freese, P. Gondolo, H. J. Newberg and M. Lewis, Phys. Rev. Lett. 92, 111301 (2004) [arXiv:astro-ph/0310334].

[41] J. D. Lewin and P. F. Smith, Astropart. Phys. 6, 87 (1996).

[42] R. W. Schnee et al. [The SuperCDMS Collaboration], arXiv:astro-ph/0502435.

[43] The parameters of the SuperCDMS experiment will be discussed in Section 4.5.

[44] We thank Richard Schnee for providing to us the results of his simulations of the capabilities of the SuperCDMS detector.

[45] J. R. Primack, D. Seckel and B. Sadoulet, Ann. Rev. Nucl. Part. Sci. 38, 751 (1988).

[46] J. L. Bourjaily and G. L. Kane, arXiv:hep-ph/0501262.

[47] G. Duda, G. Gelmini and P. Gondolo, Phys. Lett. B 529, 187 (2002) [arXiv:hep$\mathrm{ph} / 0102200]$.

[48] W. de Boer, M. Herold, C. Sander and V. Zhukov, Eur. Phys. J. C 33, S981 (2004) [arXiv:hep-ph/0312037]; W. de Boer, C. Sander, V. Zhukov, A. V. Gladyshev and D. I. Kazakov, arXiv:hep-ph/0511154, arXiv:astro-ph/0602325. 
[49] J. F. Navarro, C. S. Frenk and S. D. M. White, Astrophys. J. 462, 563 (1996) [arXiv:astro-ph/9508025].

[50] B. Moore, F. Governato, T. Quinn, J. Stadel and G. Lake, Astrophys. J. 499, L5 (1998) [arXiv:astro-ph/9709051].

[51] A. V. Kravtsov, A. A. Klypin, J. S. Bullock and J. R. Primack, Astrophys. J. 502, 48 (1998) [arXiv:astro-ph/9708176].

[52] E. A. Baltz, C. Briot, P. Salati, R. Taillet and J. Silk, Phys. Rev. D 61, 023514 (2000) [arXiv:astro-ph/9909112].

[53] J. E. Taylor and A. Babul, Mon. Not. Roy. Astron. Soc. 348, 811 (2004) [arXiv:astro-ph/0301612].

[54] Recently, the relic density calculation in the Universal Extra Dimension model of WIMP dark matter $[19,20]$ has been analyzed over a comparably large parameter space. See $[55,56]$.

[55] K. Kong and K. T. Matchev, arXiv:hep-ph/0509119.

[56] F. Burnell and G. D. Kribs, arXiv:hep-ph/0509118.

[57] J. R. Ellis, T. Falk, G. Ganis and K. A. Olive, Phys. Rev. D 62, 075010 (2000) [arXiv:hep-ph/0004169]; J. R. Ellis, K. A. Olive, Y. Santoso and V. C. Spanos, Phys. Lett. B 565, 176 (2003) [arXiv:hep-ph/0303043].

[58] J. Edsjo, M. Schelke, P. Ullio and P. Gondolo, JCAP 0304, 001 (2003) [arXiv:hep-ph/0301106].

[59] H. Baer, A. Belyaev, T. Krupovnickas and X. Tata, JHEP 0402, 007 (2004) [arXiv:hep-ph/0311351].

[60] These study points were chosen as a part of a broader study of connections between ILC measurements and cosmology, organized by J. Feng and M. Trodden. The paper is in preparation.

[61] G. Belanger, F. Boudjema, A. Pukhov and A. Semenov, Comput. Phys. Commun. 149, 103 (2002) [arXiv:hep-ph/0112278], arXiv:hep-ph/0405253.

[62] B. C. Allanach et al., in Proc. of the APS/DPF/DPB Summer Study on the Future of Particle Physics (Snowmass 2001) ed. N. Graf, Eur. Phys. J. C 25, 113 (2002) [eConf C010630, P125 (2001)] [arXiv:hep-ph/0202233].

[63] G. Weiglein et al. [LHC/LC Study Group], arXiv:hep-ph/0410364. 
[64] R. Gray et al., arXiv:hep-ex/0507008.

[65] V. Khotilovich, R. Arnowitt, B. Dutta and T. Kamon, Phys. Lett. B 618, 182 (2005) [arXiv:hep-ph/0503165].

[66] To be precise, [65] studied a set of closely related points with $m\left(\widetilde{\tau}_{1}\right)-m\left(\chi_{1}^{0}\right)=$ 4.7, 9.5, 19.0 GeV, defined with a presciption slightly different from ours for the supersymmetry spectrum calculation. Our LCC3, which has $m\left(\widetilde{\tau}_{1}\right)-m\left(\chi_{1}^{0}\right)=$ $10.5 \mathrm{GeV}$, is sufficiently close to the point 2 defined in [65] to make use of the simulations done in that study.

[67] M. Battaglia, arXiv:hep-ph/0410123.

[68] J. A. Aguilar-Saavedra et al., arXiv:hep-ph/0511344.

[69] J. L. Feng, K. T. Matchev and T. Moroi, Phys. Rev. Lett. 84, 2322 (2000) [arXiv:hep-ph/9908309], Phys. Rev. D 61, 075005 (2000) [arXiv:hep$\mathrm{ph} / 9909334$.

[70] Other studies for the LHC, for example, those reported in [71], choose additional points at much higher values of the superparticle masses. These additional points test the ability of the LHC to discover supersymmetry well beyond the region favored by natural electroweak symmetry breaking.

[71] ATLAS Collaboration, Detector and Physics Performance Technical Design Report, vol. II, CERN/LHCC/99-14 (1999).

[72] P. Bambade, M. Berggren, F. Richard and Z. Zhang, arXiv:hep-ph/0406010.

[73] G. Polesello and D. R. Tovey, JHEP 0405, 071 (2004) [arXiv:hep-ph/0403047].

[74] M. Battaglia, I. Hinchliffe and D. Tovey, J. Phys. G 30, R217 (2004) [arXiv:hep$\mathrm{ph} / 0406147]$.

[75] A. Birkedal et al., arXiv:hep-ph/0507214.

[76] W.R. Gilks, S. Richardson, D.J. Spiegelhalter, eds. Markov Chain Monte Carlo in Practice. (Chapman and Hall, 1996).

[77] J. Dunkley, M. Bucher, P. G. Ferreira, K. Moodley and C. Skordis, Mon. Not. Roy. Astron. Soc. 356, 925 (2005) [arXiv:astro-ph/0405462].

[78] E. A. Baltz and P. Gondolo, JHEP 0410, 052 (2004) [arXiv:hep-ph/0407039].

[79] B. C. Allanach and C. G. Lester, Phys. Rev. D 73, 015013 (2006) [arXiv:hep$\mathrm{ph} / 0507283]$. 
[80] R. R. de Austri, R. Trotta and L. Roszkowski, arXiv:hep-ph/0602028.

[81] A. Kusenko, P. Langacker and G. Segre, Phys. Rev. D 54, 5824 (1996) [arXiv:hep$\mathrm{ph} / 9602414]$.

[82] B. K. Gjelsten, D. J. Miller and P. Osland, arXiv:hep-ph/0507232.

[83] N. Arkani-Hamed, G. L. Kane, J. Thaler and L. T. Wang, arXiv:hep-ph/0512190.

[84] I. Hinchliffe and F. E. Paige, Phys. Rev. D 60, 095002 (1999) [arXiv:hep$\mathrm{ph} / 9812233]$.

[85] L. Bergstrom, P. Ullio and J. H. Buckley, Astropart. Phys. 9, 137 (1998) [arXiv:astro-ph/9712318].

[86] N. Gehrels and P. Michelson, Astropart. Phys. 11 (1999) 277.

[87] F. Aharonian, et al. (HESS Collaboration), Nature 439, 695 (2006).

[88] D. Hooper and B. L. Dingus, Phys. Rev. D 70, 113007 (2004) [arXiv:astro$\mathrm{ph} / 0210617]$.

[89] We thank Tsuneyoshi Kamae for discussions of these issues.

[90] P. Sreekumar et al. [EGRET Collaboration], Astrophys. J. 494, 523 (1998) [arXiv:astro-ph/9709257].

[91] K. Griest and D. Seckel, Phys. Rev. D 43, 3191 (1991).

[92] I. V. Moskalenko and A. W. Strong, Astrophys. J. 493, 694 (1998) [arXiv:astro$\mathrm{ph} / 9710124]$.

[93] E. A. Baltz and J. Edsjo, Phys. Rev. D 59, 023511 (1999) [arXiv:astroph/9808243].

[94] G. W. Bennett et al. [Muon g-2 Collaboration], Phys. Rev. Lett. 92, 161802 (2004) [arXiv:hep-ex/0401008].

[95] M. Davier, S. Eidelman, A. Hocker and Z. Zhang, Eur. Phys. J. C 27, 497 (2003) [arXiv:hep-ph/0208177].

[96] M. Davier, S. Eidelman, A. Hocker and Z. Zhang, Eur. Phys. J. C 31, 503 (2003) [arXiv:hep-ph/0308213]. 